\title{
PERTURBATIVE DEFORMATIONS OF CONFORMAL FIELD THEORIES REVISITED
}

\author{
IGOR KRIZ
}

\section{INTRODUCTION}

Recently, there has been renewed interest in the mathematics of the moduli space of conformal field theories, in particular in connection with speculations about elliptic cohomology. The purpose of this paper is to investigate this space by perturbative methods from first principles and from a purely "worldsheet" point of view. It is conjectured that at least at generic points, the moduli space of CFT's is a manifold, and in fact, its tangent space consists of marginal fields, i.e. primary fields of weight $(1,1)$ of the conformal field theory (that is in the bosonic case, in the supersymmetric case there are modifications which we will discuss later). This then means that there should exist an exponential map from the tangent space at a point to the moduli space, i.e. it should be possible to construct a continuous 1-parameter set of conformal field theories by "turning on" a given marginal field.

There is a more or less canonical mathematical procedure for applying a "Pexp" type construction to the field which has been turned on, and obtaining a perturbative expansion in the deformation parameter. This process, however, returns certain cohomological obstructions, similar to Gerstenhaber's obstructions to the existence of deformations of associative algebras [26]. Physically, these obstructions can be interpreted as changes of dimension of the deforming field, and can occur, in principle, at any order of the perturbative path. The primary obstruction is well known, and was used e.g. by Ginsparg in his work on $c=1$ conformal field theories [27. The obstruction also occured in earlier work, see [42, 43, 44, 60, 61, 62, 58, from the point of view of continuous lines in the space of critical models. In the models considered, notably the Baxter model [11, the Ashkin-Teller model [8] and the Gaussian model [45, vanishing of the primary obstruction did correspond to a continuous line of deformations, and it was therefore believed that the primary obstruction tells the whole story. (A similar story also occurs in the case of deformations of boundary sectors, see [1, 2, 12, 22, 48, 49, 55, 35.)

In a certain sense, the main point of the present paper is analyzing, or giving examples of, the role of the hihger obstructions. We shall see that these obstructions can be non-zero in cases where the deformation is believed to exist, most notably in the case of deforming the Gepner model of the Fermat quintic along a cc field, cf. $[3,52,23,57,41,63,64,14,15,17$. Some discussion of marginality of primary field in $N=2$-supersymmetric theories to higher order exists in the literature. Notably, Dixon, [19] verified the vanishing for any $N=(2,2)$-theory, and any linear combination of cc,ac,ca and ac field, of an amplitude integral which physically expresses the change of central charge (a similar calculation is also given in Distler-Greene 
[18]). Earlier work of Zamolodchikov [67, 68] showed that the renormalization $\beta$ function vanishes for theories where $c$ does not change during the renormalization process. However, we find that the calculation [19] does not guarantee that the primary field would remain marginal along the perturbative deformation path, due to subtleties involving singularities of the integral. The obstruction we discuss in this paper is an amplitude integral which physically expresses directly the change of dimension of the deforming field, and it turns out this may not vanish. We will return to this discussion in Section 3 below.

This puzzle of having obstructions where none should appear will not be fully explained in this paper, although a likely interpretation of the result will be discussed. Very likely, our effect does not impact the general question of the existence of the non-linear $\sigma$-model, which is widely believed to exist (e.g. [3, 52, 23, 57, 41, 63, 64, 14, 15, 17), but simply concerns questions of its perturbative construction. One caveat is that the case we investigate here is still not truly physical, since we specialize to the case of $c c$ fields, which are not real. The actual physical deformations of CFT's should occur along real fields, e.g. a combination of a cc field and its complex-conjugate $a a$ field (we give a discussion of this in case of the free field theory at the end of Section (4). The case of the corresponding real field in the Gepner model is much more difficult to analyze, in particular it requires regularization of the deforming parameter, and is not discussed here. Nevertheless, it is still surprising that an obstruction occurs for a single $c c$ field; for example, this does not happen in the case of the (compactified or uncompactified) free field theory.

Since an $n$ 'th order obstruction indeed means that the marginal field gets deformed into a field of non-zero weight, which changes to the order of the $n$ 'th power of the deformation parameter, usually [27, 42, 43, 44, 60, 61, 62, 58, when obstructions occur, one therefore concludes that the CFT does not possess continuous deformations in the given direction. Other interpretations are possible. One thing to observe is that our conclusion is only valid for purely perturbative theories where we assume that all fields have power series expansion in the deformation parameters with coefficients which are fields in the original theory. This is not the only possible scenario. Therefore, as remarked above, our results merely indicate that in the case when our algebraic obstruction is non-zero, non-perturbative corrections must be made to the theory to maintain the presence of marginal fields along the deformation path.

In fact, evidence in favor of this interpretation exists in the form of the analysis of Nemeschansky and Sen [52, 32 of higher order corrections to the $\beta$-function of the non-linear $\sigma$-model. Grisaru, Van de Ven and Zanon [32 found that the four-loop contribution to the $\beta$-function of the non-linear $\sigma$ model for Calabi-Yau manifolds is non-zero, and [52] found a recipe how to cancel this singularity by deforming the manifold to metric which is non-Ricci flat at higher orders of the deformation parameter. The expansion [4] used in this analysis is around the 0 curvature tensor, but assuming for the moment that a similar phenomenon occurs if we expanded around the Fermat quintic vacuum, then there are no fields present in the Gepner model which would correspond perturbatively to these higher order corrections in the direction of non-Ricci flat metric: bosonically, such fields would have to have critical conformal dimension classically, since the $\sigma$-model Lagrangian 
is classically conformally invariant for non-Ricci flat target Kähler manifolds. However, quantum mechanically, there is a one-loop correction proportional to the Ricci tensor, thus indicating that fields expressing such perturbative deformations would have to be of generalized weight (cf. [36, 37, 38, 39]). Fields of generalized weight, however, are not present in the Gepner model, which is a rational CFT, and more generally are excluded by unitarity (see discussions in Remarks after Theorems 2 , 3 in Section 3 below). Thus, although this argument is not completely mathematical, renormalization analysis seems to confirm our finding that deformations of the Fermat quintic model must in general be non-perturbative. It is also noteworthy that the $\beta$-function is known to vanish to all orders for $K 3$-surfaces because of $N=(4,4)$ supersymmetry. Accordingly, we also find that the phenomenon we see for the Fermat quintic is not present in the case of the Fermat quartic (see Section 7 below). It is also worth noting that other non-perturbative phenomena such as instanton corrections also arise when passing from $K 3$-surfaces to Calabi-Yau 3-folds ([14, 15, 17]). Finally, one must also remark that the proof of [52] of the $\beta$-function cancellation is not mathematically complete because of convergence questions, and thus one still cannot exclude even the scenario that not all non-linear $\sigma$ models would exist as exact CFT's, thus creating some type of "string landscape" picture also in this context (cf. 20]).

In this paper, we shall be mostly interested in the strictly perturbative picture. The main point of this paper is an analysis of the algebraic obstructions in certain canonical cases. We discuss two main kinds of examples, namely the free field theory (both bosonic and $N=1$-supersymmetric), and the Gepner models of the Fermat quintic and quartic, which are exactly solvable $N=2$-supersymmetric conformal field theories conjectured to be the non-linear $\sigma$-models of the Fermat quintic Calabi-Yau 3-fold and the Fermat quartic $K 3$-surface. In the case of the free field theory, what happens is essentially that all non-trivial gravitational deformations of the free field theory are algebraically obstructed. In the case of a free theory compactified on a torus, the only gravitational deformations which are algebraically unobstructed come from linear change of metric on the torus. (We will focus on gravitational deformations; there are other examples, for example the sine-Gordon interaction 66, 13, which are not discussed in detail here.)

The Gepner case deserves special attention. From the moduli space of CalabiYau 3-folds, there is supposed to be a $\sigma$-model map into the moduli space of CFT's. In fact, when we have an exactly solvable Calabi-Yau $\sigma$-model, one gets operators in CFT corresponding to the cohomology groups $H^{11}$ and $H^{21}$, which measure deformations of complex structure and Kähler metric, respectively, and these in turn give rise to infinitesimal deformations. Now the Fermat quintic

$$
x^{5}+y^{5}+z^{5}+t^{5}+u^{5}=0
$$

in $\mathbb{C} P^{4}$ has a model conjectured by Gepner [24, 25] which is embedded in the tensor product of 5 copies of the $N=2$-supersymmetric minimal model of central charge $9 / 5$. The weight $(1 / 2,1 / 2) c c$ and $a c$ fields correspond to the 100 infinitesimal deformation of complex structure and 1 infinitesimal deformation of Kähler metric of the quintic (1). Despite the numerical matches in dimension, however, it is not quite correct to say that the gravitational deformations, corresponding to the moduli space of Calabi-Yau manifolds, occurs by turning on $c c$ and $a c$ fields. This 
is because, to preserve unitarity, a physical deformation can only occur when we turn on a real field, and the fields in question are not real. In fact, the complex conjugate of a $c c$ field is an $a a$ field, and the complex conjugate of an $a c$ field is a $c a$ field. The complex conjugate must be added to get a real field, and a physical deformation (we discuss this calculationally in the case of the free field theory in Section 4).

In this paper, we do not discuss deformations of the Gepner model by turning on real fields. As shown in the case of the free field theory in Section 4 . such deformations require for example regularization of the deformation parameter, and are much more difficult to calculate. Because of this, we work with only with the case of one $c c$ and one $a c$ field. We will show that at least one $c c$ deformation, whose real version corresponds to the quintics

$$
x^{5}+y^{5}+z^{5}+t^{5}+u^{5}+\lambda x^{3} y^{2}=0
$$

for small (but not infinitesimal) $\lambda$ is algebraically obstructed. (One suspects that similar algebraic obstructions also occur for other fields, but the computation is too difficult at the moment; for the $c c$ field corresponding to $x y z t u$, there is some evidence suggesting that the deformation may exponentiate.)

It is an interesting question if non-linear $\sigma$-models of Calabi-Yau 3-folds must also contain non-perturbative terms. If so, likely, this phenomenon is generic, which could be a reason why mathematicians so far discovered so few of these conformal field theories, despite ample physical evidence of their existence [3, 52, 23, 57, 41, 63, 64.

Originally prompted by a question of Igor Frenkel, we also consider the case of the Fermat quartic $K 3$ surface

$$
x^{4}+y^{4}+z^{4}+t^{4}=0
$$

in $\mathbb{C} P^{3}$. This is done in Section [7, It is interesting that the problems of the Fermat quintic do not arise in this case, and all the infinitesimally critical fields exponentiate in the purely perturbative sense. This dovetails with the result of Alvarez-Gaume and Ginsparg [5] that the $\beta$-function vanishes to all orders for critical perturbative models with $N=(4,4)$ supersymmetry, and hence from the renormalization point of view, the non-linear $\sigma$ model is conformal for the Ricci flat metric on $K 3$-surfaces. There are also certain differences between the ways mathematical considerations of moduli space and mirror symmetry vary in the $K 3$ and Calabi-Yau 3-fold cases, which could be related to the behavior of the non-perturbative effects. This will be discussed in Section 8 .

To relate more precisely in what setup these results occur, we need to describe what kind of deformations we are considering. It is well known that one can obtain infinitesimal deformations from primary fields. In the bosonic case, the weight of these fields must be $(1,1)$, in the $N=1$-supersymmetric case in the NS-NS sector the critical weight is $(1 / 2,1 / 2)$ and in the $N=2$-supersymmetric case the infinitesimal deformations we consider are along so called ac or cc fields of weight $(1 / 2,1 / 2)$. For more specific discussion, see section 2 below. There may exist infinitesimal deformations which are not related to primary fields (see the remarks at the end of Section (3). However, they are excluded under a certain continuity assumption which we also state in section 2 . 
Therefore, the approach we follow is exponentiating infinitesimal deformations along primary fields of appropriate weights. In the "algebraic" approach, we assume that both the primary field and amplitudes can be updated at all points of the deformation parameter. Additionally, we assume one can obtain a perturbative power series expansion in the deformation parameter, and we do not allow counterterms of generalized weight or non-perturbative corrections. We describe a cohomological obstruction theory similar to Gerstenhaber's theory [26 for associative algebras, which in principle controls the coefficients at individual powers of the deformation parameter. Obstructions can be written down explicitly under certain conditions. This is done in section 3. The primary obstruction in fact is the one which occurs for the deformations of the free field theory at gravitational fields of non-zero momentum ("gravitational waves"). In the case of the Gepner model of the Fermat quintic, the primary obstruction vanishes but in the case (2), one can show there is an algebraic obstruction of order 5 (i.e given by a 7 point function in the Gepner model).

It should be pointed out that even in the "algebraic" case, there are substantial complications we must deal with. The moduli space of CFT's is not yet well defined. There are different definitions of conformal field theory, for example the Segal approach [56, 33, 34] is quite substantially different from the vertex operator approach (see [38] and references therein). Since these definitions are not known to be equivalent, and their realizations are supposed to be points of the moduli space, the space itself therefore cannot be defined until a particular definition is selected. Next, it remains to be specified what structure there should be on the moduli space. Presumably, there should at least be a topology, so than we need to ask what is a nearby conformal field theory. That, too, has not been answered.

These foundational questions are enormously difficult, mostly from the philosophical point of view: it is very easy to define ad hoc notions which immediately turn out insufficiently general to be desirable. Because of that, we only make minimal definitions needed to examine the existing paradigm in the context outlined. Let us, then, confine ourselves to observing that even in the perturbative case, the situation is not purely algebraic, and rather involves infinite sums which need to be discussed in terms of analysis. For example, the obstructions may in fact be undefined, because they may involve infinite sums which do not converge. Such phenomenon must be treated carefully, since it doesn't mean automatically that perturbative exponentiation fails. In fact, because the deformed primary fields are only determined up to a scalar factor, there is a possibility of regularization along the deformation parameter. We briefly discuss this theoretically in section 3 , and then give an example in the case of the free field theory in section 4

We also briefly discuss sufficient conditions for exponentiation. The main method we use is the case when Theorem 1 gives a truly local formula for the infinitesimal amplitude changes, which could be interpreted as an "infinitesimal isomorphism" in a special case. We then give in section 3 conditions under which such infinitesimal isomorphisms can be exponentiated. This includes the case of a coset theory, which doesn't require regularization, and a more general case when regularization may occur. 
In the final sections 5, 6, namely the case of the Gepner model, the main problem is finding a setup for the vertex operators which would be explicit enough to allow evaluating the obstructions in question; the positive result is obtained using a generalisation of the coset construction. The formulas required are obtained from the Coulomb gas approach (=Feigin-Fuchs realization), which is taken from [31].

The present paper is organized as follows: In section 2, we give the general setup in which we work, show under which condition we can restrict ourselves to deformations along a primary field, and derive the formula for infinitesimally deformed amplitudes, given in Theorem 1. In section 3, we discuss exponentiation theoretically, in terms of obstruction theory, explicit formulas for the primary and higher obstructions, and regularization. We also discuss supersymmetry, and in the end show a mechanism by which non-perturbative deformations may still be possible when algebraic obstructions occur. In section 4 we give the example of the free field theories, the trivial deformations which come from 0 momentum gravitational deforming fields, and the primary obstruction to deforming along primary fields of nonzero momentum. In section 5 , we will discuss the Gepner model of the Fermat quintic, and in section 6, we will discuss examples of non-zero algebraic obstructions to perturbative deformations in this case, as well as speculations about unobstructed deformations. In Section 7 , we will discuss the (unobstructed) deformations for the Fermat quartic $K 3$ surface, and in Section 8, we attempt to summarize and discuss our possible conclusions.

Acknowledgements: The author thanks D.Burns, I.Dolgachev, I.Frenkel, Doron Gepner, Y.Z.Huang, I.Melnikov, K.Wendland and E.Witten for explanations and discussions. Special thanks to H.Xing, who contributed many useful ideas to this project before changing his field of interest.

\section{INFINITESIMAL DEFORMATIONS OF CONFORMAL FIELD THEORIES}

In a bosonic (=non-supersymmetric) $\mathrm{CFT} \mathcal{H}$, if we have a primary field $u$ of weight $(1,1)$, then, as observed in [56, we can make an infinitesimal deformation of $\mathcal{H}$ as follows: For a worldsheet $\Sigma$ with vacuum $U_{\Sigma}$ (the worldsheet vacuum is the same thing as the "spacetime", or string, amplitude), the infinitesimal deformation of the vacuum is

$$
V_{\Sigma}=\int_{x \in \Sigma} U_{\Sigma_{u}^{x}}
$$

Here $U_{\Sigma_{u}^{x}}$ is obtained by choosing a holomorphic embedding $f: D \rightarrow \Sigma, f(0)=x$, where $D$ is the standard disk. Let $\Sigma^{\prime}$ be the worldsheet obtained by cutting $f(D)$ out of $\Sigma$, and let $U_{\Sigma_{u}^{x}}$ be obtained by gluing the vacuum $U_{\Sigma^{\prime}}$ with the field $u$ inserted at $f(\partial D)$. The element $U_{\Sigma_{u}^{x}}$ is proportional to $\left\|f^{\prime}(0)\right\|^{2}$, since $u$ is $(1,1)$-primary, so it transforms the same way as a measure and we can define the integral (31) without coupling with a measure. The integral (3) is an infinitesimal deformation of the original CFT structure in the sense that

$$
U_{\Sigma}+V_{\Sigma} \epsilon
$$

satisfies CFT gluing identities in the ring $\mathbb{C}[\epsilon] / \epsilon^{2}$. 
The main topic of this paper is studying (in this and analogous supersymmetric cases) the question as to when the infinitesimal deformation (3) can be exponentiated at least to perturbative level, i.e. when there exist for each $n \in \mathbb{N}$ elements

$$
u^{0}, \ldots, u^{n-1} \in \mathcal{H}, u^{0}=u
$$

and for every worldsheet $\Sigma$

$$
U_{\Sigma}^{0}, \ldots, U_{\Sigma}^{n} \in \bigotimes \mathcal{H}^{*} \otimes \mathcal{H}
$$

such that

$$
U_{\Sigma}(m)=\sum_{i=0}^{m} U_{\Sigma}^{i} \epsilon^{i}, U_{\Sigma}^{0}=U_{\Sigma}
$$

satisfy gluing axioms in $\mathbb{C}[\epsilon] / \epsilon^{m+1}, 0 \leq m \leq n$,

$$
u(m)=\sum_{i=0}^{m} u^{i} \epsilon^{i}
$$

is primary of weight $(1,1)$ with respect to (4), $0<m \leq n$, and

$$
\frac{d U_{\Sigma}(m)}{d \epsilon}=\int_{x \in \Sigma} U_{\Sigma_{u(m-1)}^{x}}(m-1)
$$

in the same sense as in (3).

We should remark that a priori, it is not known that all deformations of CFT come from primary fields: One could, in principle, simply ask for the existence of vacua (44) such that (44) satisfy gluing axioms over $\mathbb{C}[\epsilon] / \epsilon^{m+1}$. As remarked in [56], it is not known whether all perturbative deformations of CFT's are obtained from primary fields $u$ as describe above. However, one can indeed prove that the primary fields $u$ exist given suitable continuity assumptions. Suppose the vacua $U_{\Sigma}(m)$ exist for $0 \leq m \leq n$. We notice that the integral on the right hand side of (6) is, by definition, the limit of integrals over regions $R$ which are proper subsets of $\Sigma$ such that the measure of $\Sigma-R$ goes to 0 (fix an analytic metric on $\Sigma$ compatible with the complex structure). Let, thus, $\Sigma_{D_{1}, \ldots, D_{k}}$ be a worldsheet obtained from $\Sigma$ by cutting out disjoint holomorphically embedded copies $D_{1}, \ldots, D_{k}$ of the unit disk $D$. Then we calculate

$$
\begin{aligned}
& \frac{d U_{\Sigma}(m)}{d \epsilon}=\int_{x \in \Sigma} U_{\Sigma_{u(m-1)}^{x}}(m-1) \\
& =\lim _{\mu\left(\Sigma_{D_{1}, \ldots, D_{k}}\right) \rightarrow 0} U_{\Sigma_{D_{1}, \ldots, D_{k}}}(m-1) \int_{x \in \bigcup D_{i}} U_{\left(\bigcup D_{i}\right)_{u(m-1)}^{x}}(m-1) \\
& =\lim _{\mu\left(\Sigma_{D_{1}, \ldots, D_{k}}\right) \rightarrow 0} \sum_{i} U_{\Sigma_{D_{i}}}(m-1) \int_{x \in D_{i}} U_{\left(D_{i}\right)_{u(m-1)}^{x}}(m-1) \\
& =\lim _{\mu\left(\Sigma_{D_{1}, \ldots, D_{k}}\right) \rightarrow 0} \sum_{i} U_{\Sigma_{D_{i}}}(m-1) \frac{d U_{D_{i}}(m)}{d \epsilon}
\end{aligned}
$$


assuming (6) for $\Sigma=D$, so the assumption we need is

$$
\frac{d U_{\Sigma}}{d \epsilon}=\lim _{\mu\left(\Sigma_{D_{1}, \ldots, D_{k}}\right) \rightarrow 0} \sum_{i} U_{\Sigma_{D_{i}}}(m) \circ \frac{d U_{D_{i}}(m)}{d \epsilon} .
$$

The composition notation on the right hand side means gluing. Granted (7), we can recover $\frac{d U_{\Sigma}(m)}{d \epsilon}$ from $\frac{d U_{D}(m)}{d \epsilon}$ for the unit disk $D$. Now in the case of the unit disk, we get a candidate for $u(m-1)$ in the following way:

Assume that $\mathcal{H}$ is topologically spanned by subspaces $\mathcal{H}_{\left(m_{1}, m_{2}\right)}$ of $\epsilon$-weight $\left(m_{1}, m_{2}\right)$ where $m_{1}, m_{2} \geq 0, \mathcal{H}_{(0,0)}=\left\langle U_{D}\right\rangle$. Then $U_{D}(m)$ is invariant under rigid notation, so

$$
U_{D}(m) \in \underset{k \geq 0}{\otimes} \mathcal{H}_{(k, k)}[\epsilon] / \epsilon^{m+1}
$$

We see that if $A_{q}$ is the standard annulus with boundary components $S^{1}, q S^{1}$ with standard parametrizations, then

$$
u(m-1)=\lim _{q \rightarrow 0} \frac{1}{\|q\|^{2}} U_{A_{q}} \frac{d U_{D}(m)}{d \epsilon}
$$

exists and is equal to the weight $(1,1)$ summand of (8). In fact, by (7) and the definition of integral, we already see that (6) holds. We don't know however yet that $u(m-1)$ is primary. To see that, however, we note that for any annulus $A=D-D^{\prime}$ where $f: D \rightarrow D^{\prime}$ is a holomorphic embedding with derivative $r$, (7) also implies (for the same reason - the exhaustion principle) that (6) is valid with $u(m-1)$ replaced by

$$
\frac{U_{A} u(m-1)}{\|r\|^{2}} .
$$

Since this is true for any $\Sigma$, in particular where $\Sigma$ is any disk, the integrands must be equal, so (10) and $u(m-1)$ have the same vertex operators, so at least in the absence of null elements,

$$
\frac{U_{A} u(m-1)}{\|r\|^{2}}=u(m-1)
$$

which means that $u(m-1)$ is primary.

We shall see however that there are problems with this formulation even in the simplest possible case: Consider the free (bosonic) CFT of dimension 1, and the primary field $x_{-1} \tilde{x}_{-1}$. (We disregard here the issue that $\mathcal{H}$ itself lacks a satisfactory Hilbert space structure, see 34, we could eliminate this problem by compactifying the theory on a torus or by considering the state spaces of given momentum.) Let us calculate

$$
\begin{aligned}
& U_{D}^{1}=\int_{D} \exp \left(z L_{-1}\right) \exp \left(\bar{z} \tilde{L}_{-1}\right) x_{-1} \tilde{x}_{-1} \\
& =\frac{1}{2} \sum_{k \geq 1} \frac{x_{-k} \tilde{x}_{-k}}{k} .
\end{aligned}
$$


We see that the element (12) is not an element of $\mathcal{H}$, since its norm is $\sum_{k \geq 1} 1=\infty$. The explanation is that the 2-point function changes during the deformation, and so therefore does the inner product. Hence, if we Hilbert-complete, the Hilbert space will change as well.

For various reasons however we find this type of direct approach difficult here. For one thing, we wish to consider theories which really do not have Hilbert axiomatizations in the proper sense, including Minkowski signature theories, where the Hilbert approach is impossible for physical reasons. Therefore, we prefer a "vertex operator algebra" approach where we discard the Hilbert completion and restrict ourselves to examining tree level amplitudes. One such axiomatization of such theories was given in [38] under the term "full field algebra". In the present paper, however, we prefer to work from scratch, listing the properties we will use explicitly, and referring to our objects as conformal field theories in the vertex operator formulation.

We will then consider untopologized vector spaces

$$
V=\bigoplus V_{\left(w_{L}, w_{R}\right)} .
$$

Here $\left(w_{L}, w_{R}\right)$ are weights (we refer to $w_{L}$ resp. $w_{R}$ as the left resp. right component of the weight), so we assume $w_{L}-w_{R} \in \mathbb{Z}$ and usually

$$
\begin{gathered}
w_{L}, w_{R} \geq 0, \\
V_{(0,0)}=\left\langle U_{D}\right\rangle .
\end{gathered}
$$

The "no ghost" assumptions (14), (15) will sometimes be dropped. If there is a Hilbert space $\mathcal{H}$, then $V$ is interpreted as the "subspace of states of finite weights". We assume that for $u \in V_{w_{L}, w_{R}}$, we have vertex operators of the form

$$
Y(u, z, \bar{z})=\sum_{\left(v_{L}, v_{R}\right)} u_{-v_{L}-w_{L},-v_{R}-w_{R}} z^{v_{L}} \bar{z}^{v_{R}} .
$$

Here $u_{a, b}$ are operators which raise the left (resp. right) component of weight by $a$ (resp. b). We additionally assume $v_{L}-v_{R} \in \mathbb{Z}$ and that for a given $w$, the weights of operators which act on $w$ are discrete. Even more strongly, we assume that

$$
Y(u, z, \bar{z})=\sum_{i} Y_{i}(u, z) \tilde{Y}_{i}(u, \bar{z})
$$

where

$$
\begin{aligned}
& Y_{i}(u, z)=\sum u_{i ;-v_{L}-w_{L}} z^{v_{L}}, \\
& \tilde{Y}_{i}(u, \bar{z})=\sum \tilde{u}_{i ;-v_{R}-w_{R}} \bar{z}^{v_{R}}
\end{aligned}
$$

where all the operators $Y_{i}(u, z)$ commute with all $\tilde{Y}_{j}(v, \bar{z})$. The main axiom (16) must satisfy is "commutativity" and "associativity" analogous to the case of vertex operator algebras, i.e. there must exist for fields $u, v, w \in V$ and $w^{\prime} \in V^{\vee}$ of finite weight, a "4-point function"

$$
w^{\prime} Z(u, v, z, \bar{z}, t, \bar{t}) w
$$


which is real-analytic and unbranched outside the loci of $z=0, t=0$ and $z=t$, and whose expansion in $t$ first and $z$ second (resp. $z$ first and $t$ second, resp. $z-t$ first and $t$ second) is

$$
\begin{gathered}
w^{\prime} Y(u, z, \bar{z}) Y(v, t, \bar{t}) w, \\
w^{\prime} Y(v, t, \bar{t}) Y(u, z, \bar{z}) w, \\
w^{\prime} Y(Y(u, z-t, \overline{z-t}) v, t, \bar{t}) w,
\end{gathered}
$$

respectively. Here, for example, by an expansion in $t$ first and $z$ second we mean a series in the variable $z$ whose coefficients are series in the variable $t$, and the other cases are analogous.

We also assume that Virasoro algebras $\left\langle L_{n}\right\rangle,\left\langle\tilde{L}_{n}\right\rangle$ with equal central charges $c_{L}=c_{R}$ act and that

$$
\begin{aligned}
& Y\left(L_{-1} u, z, \bar{z}\right)=\frac{\partial}{\partial z} Y(u, z, \bar{z}), \\
& Y\left(\tilde{L}_{-1} u, z, \bar{z}\right)=\frac{\partial}{\partial \bar{z}} Y(u, z, \bar{z})
\end{aligned}
$$

and

$$
V_{w_{L}, w_{R}} \text { is the weight }\left(w_{L}, w_{R}\right) \text { subspace of }\left(L_{0}, \tilde{L}_{0}\right) \text {. }
$$

Remark: Even the axioms outlined here are meant for theories which are initial points of the proposed perturbative deformations, they are two restrictive for the theories obtained as a result of the deformations themselves. To capture those deformations, it is best to revert to Segal's approach, restricting attention to genus 0 worldsheets with a unique outbound boundary component (tree level amplitudes). Operators will then be expanded both in the weight grading and in the perturbative parameter (i.e. the coefficient at each power of the deformation parameter will be an element of the product-completed state space of the original theory). To avoid discussion of topology, we simply require that perturbative coefficients of all compositions of such operators converge in the product topology with respect to the weight grading, and the analytic topology in each graded summand.

In this section, we discuss infinitesimal perturbations, i.e. the deformed theory is defined over $\mathbb{C}[\epsilon] /\left(\epsilon^{2}\right)$ where $\epsilon$ is the deformation parameter. One case where such infinitesimal deformations can be described explicitly is the following

Theorem 1. Consider fields $u, v, w \in V$ where $u$ is primary of weight $(1,1)$. Next, assume that

$$
Z(u, v, z, \bar{z}, t, \bar{t})=\bigoplus_{\alpha, \beta} Z_{\alpha, \beta}(u, v, z, \bar{z}, t, \bar{t})
$$

where

$$
Z_{\alpha, \beta}(u, v, z, \bar{z}, t, \bar{t})=\bigoplus_{i} Z_{\alpha, \beta, i}(u, v, z, t) \tilde{Z}_{\alpha, \beta, i}(u, v, \bar{z}, \bar{t})
$$

and for $w^{\prime} \in W^{\vee}$ of finite weight, $w^{\prime} Z_{\alpha, \beta, i}(u, v, z, t)(z-t)^{\alpha} z^{\beta}$

(resp. $w^{\prime} \tilde{Z}_{\alpha, \beta, i}(u, v, \bar{z}, \bar{t}) \overline{z-t} \bar{z}^{\beta}$ ) is a meromorphic (resp. antimeromorphic) function of $z$ on $\mathbb{C} P^{1}$, with poles (if any) only at $0, t, \infty$. Now write

$$
Y_{u, \alpha, \beta}(v, t, \bar{t})=(i / 2) \int_{\Sigma} Z_{\alpha, \beta}(u, v, z, \bar{z}, t, \bar{t}) d z d \bar{z},
$$


SO

$$
Y_{u}(v, t, \bar{t})=Y(v, t, \bar{t})+\epsilon \sum_{\alpha, \beta} Y_{u, \alpha, \beta}(v, t, \bar{t})
$$

is the infinitesimally deformed vertex operator where $\Sigma$ is the degenerate worldsheet with unit disks cut out around $0, t, \infty$. Assume now further that we can expand

$$
\begin{gathered}
Z_{\alpha, \beta, i}(u, v, z, t)=Y_{\alpha, \beta, i}(v, t) Y_{\alpha, \beta, i}(u, z) \text { when } z \text { is near } 0, \\
Z_{\alpha, \beta, i}(u, v, z, t)=Y_{\alpha, \beta, i}^{\prime}(u, z) Y_{\alpha, \beta, i}(v, t) \text { when } z \text { is near } \infty \\
\left.Z_{\alpha, \beta, i}(u, v, z, t)=Y_{\alpha, \beta, i}\left(Y_{\alpha, \beta, i}^{\prime \prime}(u, z-t)\right) v, t\right) \text { when } z \text { is near } t .
\end{gathered}
$$

Write

$$
\begin{gathered}
Y_{\alpha, \beta, i}(u, z)=\sum u_{\alpha, \beta, i,-n-\beta} z^{n+\beta-1}, \\
Y_{\alpha, \beta, i}^{\prime}(u, z)=\sum u_{\alpha, \beta, i,-n-\alpha-\beta}^{\prime} z^{n+\alpha+\beta-1}, \\
Y_{\alpha, \beta, i}^{\prime \prime}(u, z)=\sum u_{\alpha, \beta, i, n-\alpha}^{\prime \prime} z^{n+\alpha-1},
\end{gathered}
$$

(Analogously with the 's.) Assume now

$$
u_{\alpha, \beta, i, 0} w=0, u_{\alpha, \beta, i, 0}^{\prime \prime} v=0, u_{\alpha, \beta, i, 0}^{\prime} Y_{\alpha, \beta, i}(v, t) w=0
$$

and analogously for the 's (note that these conditions are only nontrivial when $\beta=0$, resp. $\alpha=0$, resp. $\alpha=-\beta$ ). Denote now by $\omega_{\alpha, \beta, i, 0}, \omega_{\alpha, \beta, i, \infty}, \omega_{\alpha, \beta, i, t}$ the indefinite integrals of (23), (24), (25) in the variable $z$, obtained using the formula

$$
\int z^{k} d z=\frac{z^{k+1}}{k+1} k \neq-1
$$

(thus fixing the integration constant), and analogously with the ${ }^{\sim}$ s. Let then

$$
\begin{aligned}
& C_{\alpha, \beta, i}=\omega_{\alpha, \beta, i, \infty}-\omega_{\alpha, \beta, i, t}, \\
& D_{\alpha, \beta, i}=\omega_{\alpha, \beta, i, \infty}-\omega_{\alpha, \beta, i, 0}, \\
& \tilde{C}_{\alpha, \beta, i}=\tilde{\omega}_{\alpha, \beta, i, \infty}-\tilde{\omega}_{\alpha, \beta, i, t}, \\
& \tilde{D}_{\alpha, \beta, i}=\tilde{\omega}_{\alpha, \beta, i, \infty}-\tilde{\omega}_{\alpha, \beta, i, 0}
\end{aligned}
$$

(see the comment in the proof on branching). Let

$$
\phi_{\alpha, \beta, i}=\pi \sum_{n} \frac{u_{\alpha, \beta, i,-n} \tilde{u}_{\alpha, \beta, i,-n}}{n}
$$

where

$$
Y_{\alpha, \beta, i}(u, z)=\sum u_{\alpha, \beta, i,-n} z^{n-1}
$$

and similarly for the 's, the 's and the "'s. (The definition makes sense when applied to fields on which the term with denominator 0 vanishes.) Then

$$
\begin{aligned}
& Y_{\alpha, \beta, u}(v, t, \bar{t}) w=\sum_{i} \phi_{\alpha, \beta, i}^{\prime} Y(v, t, \bar{t}) w \\
& -Y\left(\phi_{\alpha, \beta, i}^{\prime \prime} v, t, \bar{t}\right) w-Y(v, t, \bar{t}) \phi_{\alpha, \beta, i} w+ \\
& C_{\alpha, \beta, i} \tilde{C}_{\alpha, \beta, i}\left(-1+e^{-2 \pi i \alpha}\right)+D_{\alpha, \beta, i} \tilde{D}_{\alpha, \beta, i}\left(1-e^{2 \pi i \beta}\right) .
\end{aligned}
$$


Additionally, when $\alpha=0$, then $D_{\alpha, \beta, i}=\tilde{D}_{\alpha, \beta, i}=0$, and when $\beta=0$ then $C_{\alpha, \beta, i}=$ $\tilde{C}_{\alpha, \beta, i}=0$, and

$$
Y_{\alpha, \beta, u}(v, t, \bar{t}) w=\sum_{i} \phi_{\alpha, \beta, i}^{\prime} Y(v, t, \bar{t}) w-Y\left(\phi_{\alpha, \beta, i}^{\prime \prime} v, t, \bar{t}\right) w-Y(v, t, \bar{t}) \phi_{\alpha, \beta, i} w
$$

The equation (29) is also valid when $\alpha=-\beta$.

Remark 1: Note that technically, the integral (22) is not defined on the nondegenerate worldsheet described. This can be treated in the standard way, namely by considering an actual worldsheet $\Sigma^{\prime}$ obtained by gluing on standard annuli on the boundary components. It is easily checked that if we denote by $A_{q}^{u}$ the infinitesimal deformation of $A_{q}$ by $u$, then

$$
A_{q}^{u}(w)=\phi A_{q}(w)-A_{q}(\phi w) .
$$

Therefore, the Theorem can be stated equivalently for the worldsheet $\Sigma^{\prime}$. The only change needs to be made in formula (28), where $\phi^{\prime \prime}$ needs to be multiplied by $s^{-2 n}$ and $\phi$ needs to be multiplied by $r^{-2 n}$ where $r$ and $s$ are radii of the corresponding boundary components. Because however this is equivalent, we can pretend to work on the degenerate worldsheet $\Sigma$ directly, in particular avoiding inconvenient scaling factors in the statement.

Remark 2: The validity of this Theorem is rather restricted by its assumptions. Most significantly, its assumption states that the chiral 4 point function can be rendered meromorphic in one of the variables by multiplying by a factor of the form $z^{\alpha}(z-t)^{\beta}$. This is essentially equivalent to the fusion rules being "abelian", i.e. 1-dimensional for each pair of labels, and each pair of labels has exactly one product. As we will see (and as is well known), the $N=2$ minimal model is an example of a "non-abelian" theory.

Even for an abelian theory, the theorem only calculates the deformation in the "0 charge sector" because of the assumption (26). Because of this, even for a free field theory, we will need to discuss an extension of the argument. Since in that case, however, stating precise assumptions is even more complicated, we prefer to treat the special case only, and to postpone the discussion to Section 4 below.

Proof: Let us work on the scaled real worldsheet $\Sigma^{\prime}$. Let

$$
\begin{aligned}
& \eta_{\alpha, \beta, i}=Z_{\alpha, \beta, i}(u, v, z, t) d z, \\
& \tilde{\eta}_{\alpha, \beta, i}=\tilde{Z}_{\alpha, \beta, i}(u, v, \bar{z}, \bar{t}) d \bar{z} .
\end{aligned}
$$

Denote by $\partial_{0}, \partial_{\infty}, \partial_{t}$ the boundary components of $\Sigma^{\prime}$ near $0, \infty, t$. Then the form $\omega_{\alpha, \beta, i, \infty} \tilde{\eta}_{\alpha, \beta, i}$ is unbranched on a domain obtained by making a cut $c$ connecting $\partial_{0}$ and $\partial_{t}$. We have

$$
\begin{gathered}
\oint_{\partial_{t}} \omega_{\alpha, \beta, i, t} \tilde{\eta}=-Y\left(\phi_{\alpha, \beta, i} v, t, \bar{t}\right) \\
\oint_{\partial_{0}} \omega_{\alpha, \beta, i, 0} \tilde{\eta}=-Y\left(\phi_{\alpha, \beta, i} v, t, \bar{t}\right) \phi_{\alpha, \beta, i} .
\end{gathered}
$$


But we want to integrate $\omega_{\alpha, \beta, i} \tilde{\eta}_{\alpha, \beta, i}$ over th boundary $\partial K$ :

$$
\begin{gathered}
\oint_{\partial K} \omega_{\alpha, \beta, i} \tilde{\eta}_{\alpha, \beta, i}= \\
\oint_{\partial_{t}} \omega_{\alpha, \beta, i} \tilde{\eta}_{\alpha, \beta, i}+\oint_{\partial_{0}} \omega_{\alpha, \beta, i} \tilde{\eta}_{\alpha, \beta, i}+\oint_{\partial_{\infty}} \omega_{\alpha, \beta, i} \tilde{\eta}_{\alpha, \beta, i} \\
+\int_{c^{+}} \omega_{\alpha, \beta, i} \tilde{\eta}_{\alpha, \beta, i}+\int_{c^{-}} \omega_{\alpha, \beta, i} \tilde{\eta}_{\alpha, \beta, i}
\end{gathered}
$$

where $c^{+}, c^{-}$are the two parts of $\partial K$ along the cut $c$, oriented from $\partial_{t}$ to $\partial_{0}$ and back respectively. Before going further, let us look at two points $x^{+} \in c^{+}, x^{-} \in c^{-}$ which project to the same point on $c$. We have

$$
\begin{aligned}
& C\left(e^{-2 \pi i \alpha}-1\right) \tilde{\eta}\left(x^{-}\right)= \\
& C \tilde{\eta}\left(x^{+}\right)-C \tilde{\eta}\left(x^{-}\right)=\left(\omega_{t}+C\right) \tilde{\eta}\left(x^{+}\right)-\left(\omega_{t}+C\right) \tilde{\eta}\left(x^{-}\right)= \\
& \left.\omega_{\infty} \tilde{\eta}\left(x^{+}\right)-\omega_{\infty} \tilde{\eta}\left(x^{-}\right)=\left(\omega_{0}+D\right) \tilde{\eta}\left(x^{+}\right)-\left(\omega_{o}+D\right) \tilde{(} x^{-}\right)= \\
& D \tilde{\eta}\left(x^{+}\right)-D \tilde{\eta}\left(x^{-}\right)=D\left(e^{2 \pi i \beta}-1\right) \tilde{\eta}\left(x^{-}\right)
\end{aligned}
$$

(the subscripts $\alpha, \beta, i$ were omitted throughout to simplify the notation). This implies the relation

$$
C_{\alpha, \beta, i}\left(e^{-2 \pi i \alpha}-1\right)=D_{\alpha, \beta, i}\left(e^{2 \pi i \beta}-1\right) .
$$

Comment: This is valid when the constants $C_{\alpha, \beta, i}, D_{\alpha, \beta, i}$ are both taken at the point $x^{-}$; note that since the chiral forms are branched, we would have to adjust the statement if we measured the constants elsewhere. This however will not be of much interest to us as in the present paper we are most interested in the case when the constants vanish.

In any case, note that (33) implies $C_{\alpha, \beta, i}=0$ when $\beta=0 \bmod \mathbb{Z}$ and $\alpha \neq 0$ $\bmod \mathbb{Z}$, and $D_{\alpha, \beta, i}=0$ when $\alpha=0 \bmod \mathbb{Z}$ and $\beta \neq 0 \bmod \mathbb{Z}$. There is an anlogous relation to (33) between $\tilde{C}_{\alpha, \beta, i}, \tilde{D}_{\alpha, \beta, i}$. Note that when $\alpha=0=\beta$, all the forms in sight are unbranched, and (29) follows directly. To treat the case $\alpha=-\beta$, proceed analogously, but replacing $\omega_{\alpha, \beta, i, \infty}$ by $\omega_{\alpha, \beta, i, 0}$ or $\omega_{\alpha, \beta, i, t}$. Thus, we have finished proving (29) under its hypotheses.

Returning to the general case, let us study the right hand side of (32). Subtracting the first two terms from (30), (31), we get

$$
\oint_{\partial_{t}} C_{\alpha, \beta, i} \tilde{\eta}_{\alpha, \beta, i}, \oint_{\partial_{0}} D_{\alpha, \beta, i} \tilde{\eta}_{\alpha, \beta, i}
$$

respectively. On the other hand, the sum of the last two terms, looking at points $x^{+}, x^{-}$for each $x \in c$, can be rewritten as

$$
\int_{c^{+}} C_{\alpha, \beta, i}\left(-e^{-2 \pi i \alpha}+1\right) \tilde{\eta}_{\alpha, \beta, i}=\int_{c^{-}} D_{\alpha, \beta, i}\left(-e^{2 \pi i \beta}+1\right) \tilde{\eta}_{\alpha, \beta, i}
$$

Now recall (27). Choosing $\tilde{\omega}_{\alpha, \beta, i, \infty}$ as the primitive function of $\tilde{\eta}_{\alpha, \beta, i}$, we see that for the end point $x$ of $c^{-}$,

$$
\begin{aligned}
& \tilde{\omega}_{\alpha, \beta, i, \infty}\left(x^{+}\right)-\tilde{\omega}_{\alpha, \beta, i, \infty}\left(x^{-}\right)= \\
& \tilde{\omega}_{\alpha, \beta, i, t}\left(x^{+}\right)-\tilde{\omega}_{\alpha, \beta, i, t}\left(x^{-}\right)= \\
& \left(e^{-2 \pi i \alpha}-1\right) \tilde{\omega}_{\alpha, \beta, i, t}\left(x^{-}\right)= \\
& \left(e^{-2 \pi i \alpha}-1\right) \tilde{\omega}_{\alpha, \beta, i, \infty}\left(x^{-}\right)+\left(e^{-2 \pi i \alpha}-1\right) \tilde{C}_{\alpha, \beta, i} .
\end{aligned}
$$


Similarly, for the beginning point $y$ of $c^{-}$,

$$
\begin{aligned}
& -\tilde{\omega}_{\alpha, \beta, i, \infty}\left(y^{+}\right)+\tilde{\omega}_{\alpha, \beta, i, \infty}\left(y^{-}\right)= \\
& -\tilde{\omega}_{\alpha, \beta, i, 0}\left(y^{+}\right)+\tilde{\omega}_{\alpha, \beta, i, 0}\left(y^{-}\right)= \\
& -\left(e^{2 \pi i \beta}-1\right) \tilde{\omega}_{\alpha, \beta, i, 0}\left(y^{-}\right)= \\
& -\left(e^{2 \pi i \beta}-1\right) \tilde{\omega}_{\alpha, \beta, i, \infty}\left(y^{-}\right)-\left(e^{2 \pi i \beta}-1\right) \tilde{D}_{\alpha, \beta, i} .
\end{aligned}
$$

Then (36), (37) multiplied by $C_{\alpha, \beta, i}$ are the integrals (34), while the integral (35) is

$$
-D_{\alpha, \beta, i}\left(1-e^{2 \pi i \beta}\right) \tilde{\omega}_{\alpha, \beta, i, 0}\left(y^{-}\right)+C_{\alpha, \beta, i}\left(1-e^{-2 \pi i \alpha}\right) \tilde{\omega}_{\alpha, \beta, i, 0}\left(x^{-}\right) .
$$

Adding this, we get

$$
C_{\alpha, \beta, i} \tilde{C}_{\alpha, \beta, i}\left(-1+e^{-2 \pi i \alpha}\right)+D_{\alpha, \beta, i} \tilde{D}_{\alpha, \beta, i}\left(1-e^{2 \pi i \beta}\right),
$$

as claimed.

\section{Exponentiation OF INFINITESimAL DEFORMATIONS}

Let us now look at primary weight $(1,1)$ fields $u$. We would like to investigate whether the infinitesimal deformation of vertex operators (more precisely worldsheet vacua or string amplitudes) along $u$ indeed continues to a finite deformation, or at least to perturbative level, as discussed in the previous section. Looking again at the equation (6), we see that we have in principle a series of obstructions similar to those of Gerstenhaber [26], namely if we denote by

$$
L_{n}(m)=\sum_{i=0}^{m} L_{n}^{i} \epsilon^{i}, L_{n}^{0}=L_{n}
$$

a deformation of the operator $L_{n}$ in $\operatorname{Hom}(V, V)[\epsilon] / \epsilon^{m}$, we must have

$$
\begin{gathered}
L_{n}(m) u(m)=0 \in V[\epsilon] / \epsilon^{m+1} \text { for } n>0 \\
L_{0}(m) u(m)=u(m) \in V[\epsilon] / \epsilon^{m+1} .
\end{gathered}
$$

This can be rewritten as

$$
\begin{aligned}
& L_{n} u^{m}=-\sum_{i \geq 1} L_{n}^{i} u^{m-i} \\
& \left(L_{0}-1\right) u^{m}=-\sum_{i \geq 1} L_{0}^{i} u^{m-i} .
\end{aligned}
$$

(Analogously for the "s. In the following, we will work on the obstruction for the chiral part, the antichiral part is analogous.) At first, these equations seem very overdetermined. Similarly as in the case of Gerstenhaber's obstruction theory, however, of course the obstructions are of cohomological nature. If we denote by $\mathcal{A}$ the Lie algebra $\left\langle L_{0}-1, L_{1}, L_{2}, \ldots\right\rangle$, then the system

$$
\begin{aligned}
& L_{n}(m) u(m-1) \\
& \left(L_{0}(m)-1\right) u(m-1)
\end{aligned}
$$


is divisible by $\epsilon^{m}$ in $V[\epsilon] / \epsilon^{m+1}$, and is obviously a coboundary, hence a cocycle with respect to $\left\langle L_{0}(m)-1, L_{1}(m), \ldots\right\rangle$. Hence, dividing by $\epsilon^{m}$, we get a 1 -cocycle of $\mathcal{A}$. Solving (42) means expressing this $\mathcal{A}$-cocycle as a coboundary.

In the absence of ghosts (=elements of negative weights), there is another simplification we may take advantage of. Suppose we have a 1-cocycle $c=\left(x_{0}, x_{1}, \ldots\right)$ of $\mathcal{A}$. (In our applications, we will be interested in the case when the $x_{i}$ 's are given by (42).) Then we have the equations

$$
L_{k}^{\prime} x_{j}-L_{j}^{\prime} x_{k}=(k-j) x_{j+k},
$$

where $L_{k}^{\prime}=L_{k}$ for $k>0, L_{0}^{\prime}=L_{0}-1$. In particular,

$$
L_{k}^{\prime} x_{0}-L_{0}^{\prime} x_{k}=k x_{k},
$$

or

$$
L_{k} x_{0}=\left(L_{0}+k-1\right) x_{k} \text { for } k>0 .
$$

In the absence of ghosts, (44) means that for $k \geq 1, x_{k}$ is determined by $x_{0}$ with the exception of the weight 0 summand $\left(x_{1}\right)_{0}$ of $x_{1}$. Additionally, if we denote the weight $k$ summand of $y$ in general by $y_{k}$, then

$$
c=d y
$$

means

$$
\begin{gathered}
\left(x_{0}\right)_{k}=(k-1) y, \\
\left(x_{0}\right)_{1}=0 .
\end{gathered}
$$

The rest of the equation (45) then follows from (44), with the exception of the weight 0 summand of $x_{1}$. We must, then, have

$$
\left(x_{1}\right)_{0} \in \operatorname{Im} L_{1} .
$$

Conditions (47), (48), for

$$
x_{k}=-\sum_{i \geq 1} L_{k}^{i} u^{m-i},
$$

are the conditions for solving (42), i.e. the actual obstruction.

For $m=1$, we get what we call the primary obstruction. We have

$$
L_{k}^{1}=\tilde{L}_{-k}^{1}=\sum_{m, i} u_{i, m+k} \tilde{u}_{i, m},
$$

so (47) becomes

$$
\sum_{i} u_{i, 0} \tilde{u}_{i, 0} u=0 .
$$

The condition (48) becomes

$$
\sum_{i} u_{i, 1} \tilde{u}_{i, 0} u \in \operatorname{Im} L_{1}, \quad \sum_{i} u_{i, 0} \tilde{u}_{i, 1} u \in \operatorname{Im} \tilde{L}_{1} .
$$


This investigation is also interesting in the supersymmetric context. In the case of $N=1$ worldsheet supersymmetry, we have additional operators $G_{r}^{i}$, and in the $N=2$ SUSY case, we have operators $G_{r}^{+i}, G_{r}^{-i}, J_{n}^{i}$ (cf. [28, 46]), defined as the $\epsilon^{i}$-coefficient of the deformation of $G_{r}$, resp. $G_{r}^{+}, G_{r}^{-}, J_{n}$ analogously to equation (39).

In the $N=1$-supersymmetric case, the critical deforming fields have weight $(1 / 2,1 / 2)$ (as do $a$ - and $c$-fields in the $N=2$ case), so in both cases the first equation (39) remains the same as in the $N=0$ case, the second becomes

$$
\left(L_{0}-1 / 2\right) u^{m}=-\sum_{i \geq 1} L_{0}^{i} u^{m-i} .
$$

Additionally, for $N=1$, we get

$$
G_{r} u^{m}=-\sum_{i \geq 1} G_{r}^{i} u^{m-i}, r \geq 1 / 2
$$

(similarly when $\sim$ s are present).

In the $N=1$-supersymmetric case, we therefore deal with the Lie algebra $\mathcal{A}$, which is the free $\mathbb{C}$-vector space on $L_{n}, G_{r}, n \geq 0, r \geq 1 / 2$. For a cocycle which has value $x_{k}$ on $L_{k}$ and $z_{r}$ on $G_{r}$, the equation (44) becomes

$$
L_{k} x_{0}=\left(L_{0}+k-1 / 2\right) x_{k} \text { for } k>0,
$$

so in the absence of ghosts, $x_{k}$ is always determined by $x_{0}$. If

$$
\text { the 1-cocycle }\left(x_{k}, z_{r}\right) \text { is the coboundary of } y
$$

we additionally get

$$
\left(x_{0}\right)_{k}=(k-1 / 2) y,
$$

SO

$$
\left(x_{0}\right)_{1 / 2}=0 .
$$

On the other hand, on the $z$ 's, we get

$$
G_{r} x_{0}=\left(L_{0}+r-1 / 2\right) z_{r}, r \geq 1 / 2,
$$

so we see that in the absence of ghosts, all $z_{r}$ 's are determined, with the exception of

Therefore our obstruction is

$$
\left(z_{1 / 2}\right)_{0}
$$

$$
\left(z_{0}\right)_{1 / 2}=0,\left(z_{1 / 2}\right)_{0} \in \operatorname{Im}\left(G_{1 / 2}\right) .
$$

For the primary obstruction, we have

$$
\begin{aligned}
L_{k}^{1}=\tilde{L}_{-k}^{1} & \left.=\sum_{m}\left(G_{-1 / 2} \tilde{G}_{-1 / 2} u\right)_{m+k, m}\right), \\
G_{r}^{1} & =2 \sum_{m}\left(\tilde{G}_{-1 / 2} u\right)_{m+r, m}, \\
\tilde{G}_{r}^{1} & =2 \sum_{m}\left(G_{-1 / 2} u\right)_{m, m+r},
\end{aligned}
$$


so the obstruction becomes

$$
\begin{aligned}
& \sum_{m}\left(G_{-1 / 2} \tilde{G}_{-1 / 2} u\right)_{m, m}=0, \\
& \sum_{m}\left(\tilde{G}_{-1 / 2} u\right)_{m+1 / 2, m} \in \operatorname{Im}\left(G_{1 / 2}\right), \\
& \sum_{m}\left(G_{-1 / 2} u\right)_{m, m+1 / 2} \in \operatorname{Im}\left(\tilde{G}_{1 / 2}\right) .
\end{aligned}
$$

In the case of $N=2$ supersymmetry, there is an additional complication, namely chirality. This means that in addition to the conditions

$$
\begin{aligned}
& \left(L_{0}-1 / 2\right) u=0, \\
& L_{n} G_{r}^{ \pm} u=J_{n-1}=0 \text { for } n \geq 1, r \geq 1 / 2,
\end{aligned}
$$

we require that $u$ be chiral primary, which means

$$
G_{-1 / 2}^{+} u=0 .
$$

(There is also the possibility of antichiral primary, which has

$$
G_{-1 / 2}^{-} u=0
$$

instead, and similarly at the “s.) Let us now write down the obstruction equations for the chiral primary case. We get the first equation (42), (51), and an analogue of (52) with $G_{r}^{i}$ replaced by $G_{r}^{+i}$ and $G_{r}^{-i}$. Additionally, we have the equation

$$
G_{-1 / 2}^{+} u^{m}=-\sum_{i \geq 1} G_{-1 / 2}^{i} u^{m-i}
$$

and analogously for the "s.

In this situation, we consider the super-Lie algebra $\mathcal{A}_{2}$ which is the free $\mathbb{C}$-vector space on $L_{n}, J_{n}, n \geq 0, G_{r}^{-}, r \geq 1 / 2$ and $G_{s}^{+}, s \geq-1 / 2$. One easily verifies that this is a super-Lie algebra on which the central extension vanishes canonically ([28, Section 3.1). Looking at a 1-cocycle whose value is $x_{k}, z_{r}^{ \pm}, t_{k}$ on $L_{k}, G_{r}^{ \pm}, J_{k}$ respectively, we get the equation (53), and additionally

$$
G_{r}^{ \pm} x_{0}=\left(L_{0}+r-1 / 2\right) z_{r}^{ \pm}, r \geq 1 / 2 \text { for }-, r \geq-1 / 2 \text { for }+
$$

and

$$
J_{n} x_{0}=(n-1 / 2) t_{n}, n \geq 0 .
$$

We see that the cocycle is determined by $x_{0}$, with the exception of $\left(z_{1 / 2}^{ \pm}\right)_{0},\left(z_{-1 / 2}^{+}\right)_{1}$. Therefore, we get the condition

$$
\begin{aligned}
& \left(x_{0}\right)_{1 / 2}=0 \\
& \left(z_{1 / 2}^{ \pm}\right)_{0} \in \operatorname{Im}\left(G_{1 / 2}^{ \pm}\right) \\
& \left(z_{-1 / 2}^{+}\right)_{1}=G_{-1 / 2}^{+} u \text { where } G_{1 / 2}^{+} u=0
\end{aligned}
$$

and similarly for the "s. 
In the case of deformation along a $c c$ field $u$, we have

$$
\begin{gathered}
L_{k}^{1}=\tilde{L}_{-k}^{1}=\sum_{m}\left(G_{-1 / 2}^{-} \tilde{G}_{-1 / 2}^{-} u\right)_{m+k, m}, \\
G_{r}^{+, 1}=\sum_{m} 2\left(\tilde{G}_{-1 / 2}^{-} u\right)_{m+r+1 / 2, m} \\
\tilde{G}_{r}^{+, 1}=\sum_{m} 2\left(G_{-1 / 2}^{-} u\right)_{m, m+r+1 / 2} \\
G_{r}^{-, 1}=\tilde{G}_{r}^{-, 1}=0 \\
J_{n}^{1}=0=\tilde{J}_{n}^{1}
\end{gathered}
$$

so the obstructions are, in a sense, analogous to (59) with $G_{r}$ replaced by $G_{r}^{-}$.

Remark: The relevant computation in verifying that (66), (67) (and the analogous cases before) form a cocycle uses formulas of the following type ([69]):

$$
\operatorname{Res}_{z}\left(a(z) v(w) z^{n}\right)-\operatorname{Res}_{z}\left(v(w) a(z) z^{n}\right)=\operatorname{Res}_{z-w}\left((a(z-w) v)(w) z^{n}\right) .
$$

For example, when $v$ is primary of weight $1, a=L_{-2}$, the right hand side of (68) is

$$
\begin{aligned}
& \operatorname{Res}_{z-w}\left(L_{0} v(z-w)^{-2} n(z-w) w^{n-1}+L_{-1} v(z-w)^{-1} w^{n}\right) \\
& =n v(w) w^{n-1}+L_{-1} v(w) w^{n} \\
& =\sum n v_{k} w^{n-k-2}+\sum_{k}(-k-1) v_{k} w^{n-k-2} \\
& =\sum(n-k) v_{n} w^{n-k-2} .
\end{aligned}
$$

The left hand side is $\sum\left[L_{n-1}, v_{k-n+1}\right] w^{n-k-2}$, so we get

$$
\left[L_{n-1}, v_{k-n+1}\right]=(n-k-1) v_{k},
$$

as needed.

Other required identities follow in a similar way. Let us verify one interesting case when $a=G_{-3 / 2}^{-1}, u$ chiral primary. Then the right hand side of (68) is

$$
\operatorname{Res}_{z-w}\left(G_{-1 / 2}^{-} v(w)(z-w)^{-1} w^{n}\right)=\left(G_{-1 / 2}^{-} v\right)(w)=\sum\left(G_{-1 / 2}^{-} v\right) w^{-n-1} .
$$

This implies

$$
\left[G_{r}^{-}, u_{s}\right]=\left(G_{-1 / 2}^{-} u\right)_{r+s}
$$

as needed.

We have now analyzed the primary obstructions for exponentiation of infinitesimal CFT deformations. However, in order for a perturbative exponentiation to exist, there are also higher obstructions which must vanish. The basic principle for obtaining these obstructions was formulated above. However, in pratice, it may often happen that those obstructions will not converge. This may happen for two different basic reasons. One possibility is that the deformation of the deforming field itself does not converge. This is essentially a violation of perturbativity, but may in some cases be resolved by regularizing the CFT anomaly along the deformation parameter. We will discuss this at the end of this section, and will give an example in Section 4 below. 
Even if all goes well with the parameter, however, there may be another problem, namely the expressions for $L_{n}^{i}$ etc. may not converge due to the fact that our deformation formulas concern vacua of actual worldsheets, while $L_{n}^{i}$ etc. correspond to degenerate worldsheets. Similarly, vertex operators may not converge in the deformed theories. We will show here how to deal with this problem.

The main strategy is to rephrase the conditions from the above part of this section in terms of "finite annuli". We start with the $N=0$ (non-supersymmetric) case. Similarly as in (39), we can expand

$$
U_{A_{r}}(m)=\sum_{h=0}^{m} U_{A_{r}}^{h} \epsilon^{h} .
$$

In the non-supersymmetric case, the basic fact we have is the following:

Theorem 2. Assuming $u^{k}$ (considered as fields in the original undeformed CFT) have weight $>(1,1)$ for $k<h, r \in(0,1)$ we have

$$
\begin{gathered}
U_{A_{r}}^{h}=\sum_{\sum_{s_{h-1}=r}} \int_{s_{h}=r}^{1} s_{h}^{2 m_{h}-1} \int_{s_{1}}^{s_{h}} s_{h-1}^{2 m_{h-1}-1} \ldots \\
\int_{s_{1}=r}^{s_{2}} s_{1}^{2 m_{1}-1} u_{m_{h}, m_{h}} \ldots u_{m_{1}, m_{1}} d s_{1} \ldots d s_{h} U_{A_{r}} . \\
u^{h}=\sum_{m_{k}} \frac{1}{2^{h}\left(m_{h}+\ldots+m_{1}\right)\left(m_{h-1}+\ldots+m_{1}\right) \ldots m_{1}} u_{m_{h}, m_{h} \ldots u_{m_{1}, m_{1}} u .}
\end{gathered}
$$

In particular, the obstruction is the vanishing of the sum (with the term $m_{h}+\ldots m_{1}$ omitted from the denominator) of the terms in (72) with $m_{h}+\ldots m_{1}=0$.

Proof: The identity (71) is essentially by definition. The key point is that in the higher deformed vacua, there are terms in the integrand obtained by inserting $u_{k}$, $k>1$ to boundaries of disjoint disks $D_{i}$ cut out of $A_{r}$. Then there are corrective terms to be integrated on the worldsheets obtained by cutting out those disks. But the point is that under our weight assumption, all the disks $D_{i}$ can be shrunk to a single point, at which point the term disappears, and we are left with integrals of several copies of $u$ inserted at different points. If we are using vertex operators to express the integral, the operators must additionally be applied in time order (i.e. fields at points of lower modulus are inserted first). There is an $h$ ! permutation factor which cancels with the Taylor denominator. This gives (71).

Now (72) is proved by induction. For $h=1$, the calculation is done above. Assuming the induction hypothesis, the term of the integral where the $k-1$ innermost integrals have the upper bound and the $k$ 'th innermost integral has the lower bound is equal to

$$
U_{A_{r}}^{h-k} u^{k}, h>k \geq 1 \text {. }
$$

The summand which has all upper bounds except in the last integral is equal to

$$
\frac{1-r^{2\left(m_{1}+\ldots+m_{h}\right)}}{2^{h}\left(m_{h}+\ldots+m_{1}\right)\left(m_{h-1}+\ldots+m_{1}\right) \ldots m_{1}} u_{m_{h}, m_{h}} \ldots u_{m_{1}, m_{1}} u r^{2}
$$


which is supposed to be equal to

$$
-U_{A_{r}} u^{h}+r^{2} u^{h}
$$

This gives the desired solution.

Remark: The formula (73) of course does not apply to the case $m_{1}+\ldots+m_{h}=0$. In that case, the correct formula is

$$
\frac{-\ln (r)}{\left(m_{h-1}+\ldots+m_{1}\right) \ldots m_{1}} u_{m_{h}, m_{h}} \ldots u_{m_{1}, m_{1}} u r^{2} .
$$

So the question becomes whether there could exist a field $u^{h}$ such that $U_{A_{r}} u^{h}-r^{2} u^{h}$ is equal to the quantity (74). One sees immediately that such field does not exist in the product-completed space of the original theory. What this approach does not settle however is whether it may be possible to add such non-perturbative fields to the theory and preserve CFT axioms, which could facilitate existence of deformations in some generalized sense, despite the algebraic obstruction. It would have to be, however, a field of generalized weight in the sense of [36, 37, 38, 39.

In effect, written in infinitesimal terms, the relation (74) becomes

$$
L_{0} u^{h}-u^{h}=-\frac{1}{\left(m_{h-1}+\ldots+m_{1}\right) \ldots m_{1} u_{m_{h}, m_{h}}} \ldots u_{m_{1}, m_{1}} u .
$$

The right hand side $w$ is a field of holomorphic weight 1 , so we see that we have a matrix relation

$$
L_{0}\left(\begin{array}{l}
u^{h} \\
u
\end{array}\right)=\left(\begin{array}{cc}
1 & w \\
0 & 1
\end{array}\right)\left(\begin{array}{l}
u^{h} \\
u
\end{array}\right)
$$

This is an example of what one means by a field of generalized weight. One should note, however, that fields of generalized weight are excluded in unitary conformal field theories. By Wick rotation, the unitary axiom of a conformal field theory becomes the axiom of reflection positivity [56]: the operator $U_{\Sigma}$ associated with a worldsheet $\Sigma$ is defined up to a 1-dimensional complex line $L_{\Sigma}$ (which is often more strongly assumed to have a positive real structure). If we denote by $\bar{\Sigma}$ the complexconjugate worldsheet (note that this reverses orientation of boundary components), then reflection positivity requires that we have an isomorphism $L_{\overline{\text { Sigma }}} \cong L_{\Sigma}^{*}$ (the dual line), and using this isomorphism, an identification $U_{\bar{\Sigma}}=U_{\Sigma}^{*}$ (here the asterisk denotes the adjoint operator). Specializing to annuli $A_{r},\|r\| \leq 1$, we see that the annulus for $r$ real is self-conjugate, so the corresponding operators are selfadjoint, and hence diagonalizable. On the other hand, for $\|r\|=1$, we obtain unitary operators, and unitary representations of $S^{1}$ on Hilbert space split into eigenspaces of integral weights. The central extension given by $L$ is then trivial and hence the operators corresponding to all $A_{r}$ commute, and hence are simultaneously diagonalizable, thus excluding the possibility of generalized weight.

The possibility, of course, remains that the correlation function of the deformed theory can be modified by a non-perturbative correction. Let us note that if left uncorrected, the term (74) can be interpreted infinitesimally as

$$
L_{0} u(\epsilon)-u(\epsilon)=C \epsilon^{m} v \bmod \epsilon^{m+1},
$$

where $v$ is another field of weight 1 . Note that in case that $u=v$, (75) can be interpreted as saying that $u$ changes weight at order $m$ of the perturbation parameter. In the general case, we obtain a matrix involving all the (holomorphic) 
weight 1 fields in the unperturbed theory. Excluding fields of generalized weight in the unperturbed theory (which would translate to fields of generalized weight in the perturbed theory), the matrix must have other eigenvalues than 1 , thus showing that some critical fields will change weight.

In the $N=1$-supersymmetric case, an analogous statement holds, except the assumption is that the weight of $u^{k}$ is greater than $(1 / 2,1 / 2)$ for $k<h$, and the integral (71) must be replaced by

$$
\begin{aligned}
U_{A_{r}}^{h}= & \sum_{m_{k}} \int_{s_{h}=r}^{1} s_{h}^{m_{h}-1} \int_{s_{h-1}=r}^{s_{h}} s_{h-1}^{m_{h-1}-1} \ldots \\
s_{2} & s_{1}^{m_{1}-1}\left(G_{-1 / 2} \tilde{G}_{-1 / 2} u\right)_{m_{h}, m_{h}} \ldots\left(G_{-1 / 2} \tilde{G}_{-1 / 2} u\right)_{m_{1}, m_{1}} d s_{1} \ldots d s_{h} U_{A_{r}},
\end{aligned}
$$

and accordingly

$$
\begin{aligned}
& u^{h}=\sum_{m_{k}} \frac{1}{2^{h}\left(m_{h}+\ldots+m_{1}\right)\left(m_{h-1}+\ldots+m_{1}\right) \ldots m_{1}} \\
& \left(G_{-1 / 2} \tilde{G}_{-1 / 2} u\right)_{m_{h}, m_{h} \ldots\left(G_{-1 / 2} \tilde{G}_{-1 / 2} u\right)_{m_{1}, m_{1}} u,}
\end{aligned}
$$

so the obstruction again states that the term with $m_{h}+\ldots+m_{1}=0$ must vanish. In the $N=2$ case, when $u$ is a $c c$ field, we simply replace $G$ by $G^{-}$in (76), (77).

But in the supersymmetric case, to preserve supersymmetry along the deformation, we must also investigate the "finite" analogs of the obstructions associated with $G_{1 / 2}$ in the $N=1$ case, and $G_{1 / 2}^{ \pm}, G_{-1 / 2}^{+}$in the $N=2 c$ case (and similarly for the $a$ case, and the ${ }^{\sim}$ 's). In fact, to tell the whole story, we should seriously investigate integration of the deforming fields over super-Riemann surfaces (=superworldsheets). This can be done; one approach is to treat the case of the superdisk first, using Stokes theorem twice with the differentials $\partial, \bar{\partial}$ replaced by $D, \bar{D}$ respectively in the $N=1$ case (and the same at one chirality for the $N=2$ case). A general super-Riemann surface is then partitioned into superdisks.

For the purpose of obstruction theory, the following special case is sufficient. We treat the $N=2$ case, since it is of main interest for us. Let us consider the case of $c c$ fields (the other cases are analogous). First we note (see (67)) that $G^{-}$is unaffected by deformation via a $c c$ field, so the obstructions derived from $G_{-1 / 2}^{-}$ and $G_{1 / 2}^{-}$are trivial (and similarly at the "s).

To understand the obstruction associated with $G_{1 / 2}^{+}$, we will study "finite" (as opposed to infinitesimal) annuli obtained by exponentiating $G_{1 / 2}^{+}$. Now the element $G_{1 / 2}^{+}$is odd. Thinking of the super-semigroup of superannuli as a supermanifold, then it makes no sense to speak of "odd points" of the supermanifold. It makes sense, however, to speak of a family of edd elements parametrized by an odd parameter $s$ : this is simply the same thing as a map from the (0|1)-dimensional superaffine line into the supermanifold. In this sense, we can speak of the "finite" odd annulus

$$
\exp \left(s G_{1 / 2}^{+}\right)
$$


Now we wish to study the deformations of teh operator associated with (78) along a $c c$ field $u$ as a perturbative expansion in $\epsilon$.

Thinking of $G_{1 / 2}^{+}$as an $N=2$-supervector field, we have

$$
G_{1 / 2}^{+}=\left(z+\theta^{+} \theta^{-}\right) \frac{\partial}{\partial \theta^{+}}-z \theta^{-} \frac{\partial}{\partial z} .
$$

We see that (79) deforms infinitesimally only the variables $\theta^{+}$and $z$, not $\theta^{-}$. Thus, more specifically, (78) results in the transformation

$$
\begin{aligned}
& z \mapsto \exp \left(s \theta^{-}\right) z \\
& \theta^{-} \mapsto \theta^{-} .
\end{aligned}
$$

This gives rise to the formula, valid when $u^{k}$ have weight $>(1 / 2,1 / 2)$ for $1 \leq k<h$,

$$
\begin{aligned}
& U_{\exp \left(s G_{1 / 2}^{+}\right)}^{h}= \\
& \sum_{m_{k}} \int_{t_{h}=\exp \left(s \theta^{-}\right)}^{1} t_{h}^{m_{h}-1} \int_{t_{h-1}=\exp \left(s \theta^{-}\right)}^{t_{h}} t_{h-1}^{m_{h-1}-1} \ldots \\
& \left.\int_{t_{1}=\exp \left(s \theta^{-}\right)}^{t_{2}} s_{1}^{m_{1}-1} v_{m_{h}, m_{h}} \ldots v_{m_{1}, m_{1}} d t_{1} \ldots d t_{h} U_{\exp \left(s G_{1 / 2}^{+}\right)}\right)
\end{aligned}
$$

where $v_{m_{k}, m_{k}}$ is equal to

$$
\left(\tilde{G}_{-1 / 2}^{-} u\right)_{m_{k+1 / 2}, m_{k}}
$$

in summands of (81) where the factor resulting from integrating the $t_{k}$ variable has a $\theta^{-}$factor, and

$$
\left(G_{-1 / 2}^{-} \tilde{G}_{-1 / 2}^{-} u\right)_{m_{k}, m_{k}}
$$

in other summands. (We see that each summand can be considered as a product of factors resulting from integrating the individual variables $t_{k}$; in at most one factor, (82) can occur, otherwise the product vanishes.)

Realizing that $\exp \left(m s \theta^{-}\right)=1+m s \theta^{-}$, this gives that the obstruction (under the weight assumption for $u^{k}$ ) is that the summand for $m_{1}+\ldots+m_{h}=0$ (with the denominator $m_{1}+\ldots+m_{h}$ omitted) in the following expression vanish:

$$
\begin{aligned}
& \sum_{m_{k}} \sum_{k=1}^{h} \frac{1}{m_{1}+\ldots+m_{h}} \cdots \frac{1}{m_{1}} \\
& \left(G_{-1 / 2}^{-} \tilde{G}_{-1 / 2}^{-} u\right)_{m_{h}, m_{h}} \ldots m_{k}\left(\tilde{G}_{-1 / 2}^{-} u\right)_{m_{k+1 / 2}, m_{k}} \ldots\left(G_{-1 / 2}^{-} \tilde{G}_{-1 / 2}^{-} u\right)_{m_{1}, m_{1}} u .
\end{aligned}
$$

To investigate the higher obstructions further, we need the language of correlation functions. Specifically, the CFT's whose deformations we will consider are "RCFT's". The simplest way of building an RCFT is from "chiral sectors" $\mathcal{H}_{\lambda}$ where $\lambda$ runs through a set of labels, by the recipe

$$
\mathcal{H}=\underset{\lambda}{\bigoplus} \mathcal{H}_{\lambda} \otimes \mathcal{H}_{\lambda^{*}}
$$

where $\lambda^{*}$ denotes the contragredient label (cf. 35]). (In the case of the Gepner model, we will need a slightly more general scenario, but our methods still apply 
to that case analogously.) Further, we will have a symmetric bilinear form

$$
B: \mathcal{H}_{\lambda} \otimes \mathcal{H}_{\lambda^{*}} \rightarrow \mathbb{C}
$$

with respect to which the adjoint to $Y(v, z)$ is

$$
\left(-z^{-2}\right)^{n} Y\left(e^{z L_{1}} v, 1 / z\right)
$$

where $v$ is of weight $n$. There is also a real structure

$$
\mathcal{H}_{\lambda} \cong \overline{\mathcal{H}}_{\lambda^{*}}
$$

thus specifying a real structure on $\mathcal{H}, \overline{u \otimes v}=\bar{u} \otimes \bar{v}$, and inner product

$$
\left\langle u_{1} \otimes v_{1}, u_{2} \otimes v_{2}\right\rangle=B\left(u_{1}, \overline{u_{2}}\right) B\left(v_{1}, \overline{v_{2}}\right) .
$$

We also have an inner product

$$
\mathcal{H}_{\lambda} \otimes_{\mathbb{R}} \mathcal{H}_{\lambda^{*}} \rightarrow \mathbb{C}
$$

given by

$$
\langle u, v\rangle=B(u, \bar{v}) .
$$

Then we have the $\mathbb{P}^{1}$-chiral correlation function

$$
\left\langle u\left(z_{\infty}\right)^{*} \mid v_{m}\left(z_{m}\right) v_{m-1}\left(z_{m-1}\right) \ldots v_{1}\left(z_{1}\right) v_{0}\left(z_{0}\right)\right\rangle
$$

which can be defined by taking the vacuum operator associated with the degenerate worldsheet $\Sigma$ obtained by "cutting out" unit disks with centers $z_{0}, \ldots, z_{m}$ from the unit disk with center $z_{\infty}$, applying this operator to $v_{0} \otimes \ldots \otimes v_{m}$, and taking inner product with $u$. Thus, the correlation function (85) is in fact the same thing as applying the field on either side of (85) to the identity, and taking the inner product.

This object (85) is however not simply a function of $z_{0}, \ldots, z_{\infty}$. Instead, there is a finite-dimensional vector space $M_{\Sigma}$ depending holomorphically on $\Sigma$ (called the modular functor) such that (85) is a linear function

$$
M_{\Sigma} \rightarrow \mathbb{C} .
$$

However, now one assumes that $M$ is a "unitary modular functor" in the sense of Segal [56. This means that $M_{\Sigma}$ has the structure of a positive-definite inner product space for not just the $\Sigma$ as above, but an arbitrary worldsheet. The inner product is not valued in $\mathbb{C}$, but in

$$
\|\operatorname{det}(\Sigma)\|^{2 c}
$$

where $c$ is the central charge. Since the determinant of $\Sigma$ as above is the same as $\operatorname{det}\left(\mathbb{P}^{1}\right)$ (hence in particular constant), we can make the inner product $\mathbb{C}$-valued in our case.

If the deforming field is of the form

$$
u \otimes \tilde{u},
$$

the "higher $L_{0}$ obstruction" (under the weight assumptions given above) can be further written as

$$
\begin{aligned}
& \int_{0 \leq\left\|z_{1}\right\| \leq\left\|z_{m}\right\| \leq 1}\left\langle v(0)^{*} \mid u\left(z_{m}\right) \ldots u\left(z_{1}\right) u(0)\right\rangle \\
& \left\langle\tilde{v}^{*} \mid \tilde{u}\left(\overline{z_{m}}\right) . . \tilde{u}\left(\overline{z_{1}}\right) \tilde{u}(0)\right\rangle d z_{1} d \bar{z}_{1} \ldots . d z_{m} d \bar{z}_{m} \\
& \text { for } w(v) \leq 1
\end{aligned}
$$


( $w$ is weight) in the $N=0$ case and

$$
\begin{aligned}
& \int_{0 \leq\left\|z_{1}\right\| \leq\left\|z_{m}\right\| \leq 1}\left\langle v(0)^{*} \mid\left(G_{-1 / 2}^{-} u\right)\left(z_{m}\right) \ldots\left(G_{-1 / 2}^{-} u\right)\left(z_{1}\right) u(0)\right\rangle \\
& \left\langle\tilde{v}^{*} \mid\left(\tilde{G}_{-1 / 2}^{-} \tilde{u}\right)\left(\overline{z_{m}}\right) \ldots\left(\tilde{G}_{-1 / 2}^{-} \tilde{u}\right)\left(\overline{z_{1}}\right) \tilde{u}(0)\right\rangle d z_{1} d \bar{z}_{1} \ldots . d z_{m} d \bar{z}_{m} \\
& \text { for } w(v) \leq 1 / 2
\end{aligned}
$$

in the $N=2 c c$ case. The $G_{1 / 2}^{+}$-obstruction in the $N=2$ case can be written as

$$
\begin{aligned}
& \int_{0 \leq\left\|z_{1}\right\| \leq\left\|z_{m}\right\| \leq 1} \sum_{k=1}^{m}\left\langle v(0)^{*} \mid\left(G_{-1 / 2}^{-} u\right)\left(z_{m}\right) \ldots u\left(z_{k}\right) \ldots\left(G_{-1 / 2}^{-} u\right)\left(z_{1}\right) u(0)\right\rangle \\
& \left\langle\tilde{v}^{*} \mid\left(\tilde{G}_{-1 / 2}^{-} \tilde{u}\right)\left(\overline{z_{m}}\right) \ldots\left(\tilde{G}_{-1 / 2}^{-} \tilde{u}\right)\left(\overline{z_{1}}\right) \tilde{u}(0)\right\rangle \\
& d z_{1} d \bar{z}_{1} \ldots . d z_{m} d \bar{z}_{m} \text { for } w(v) \leq 0, w(\tilde{v}) \leq 1 / 2
\end{aligned}
$$

and similarly for the . We see that these obstructions vanish when we have

$$
\left\langle v\left(z_{\infty}\right)^{*} \mid u\left(z_{m}\right) \ldots u\left(z_{0}\right)\right\rangle=0, \text { for } w(v) \leq 1
$$

in the $N=0$ case (and similarly for the "s), and

$$
\left\langle v\left(z_{\infty}\right)^{*} \mid G_{-1 / 2}^{-} u\left(z_{m}\right) \ldots . G_{-1 / 2}^{-} u\left(z_{1}\right) u\left(z_{0}\right)\right\rangle=0, \text { for } w(v) \leq 1 / 2,
$$

and similarly for the "s. Observe further that when

$$
\tilde{u}=\bar{u},
$$

the condition for the "s is equivalent to the condition for $u$, and further (90), (91) are also necessary in this case, as in (87), (88) we may also choose $\tilde{v}=\bar{v}$, which makes the integrand non-negative (and only 0 if it is 0 at each chirality). In the $N=2$ case, it turns the condition (91) simplifies further:

Theorem 3. Let $u$ be a chiral primary field of weight $1 / 2$. Then the necessary and sufficient condition (91) for existence of perturbative CFT deformations along the field $u \otimes \bar{u}$ is equivalent to the same vanishing condition applied to only chiral primary fields $v$ of weight $1 / 2$.

\section{Proof:}

In order for the fields (91) to correlate, they would have to have the same $J$ charge $Q_{J}$. We have

$$
Q_{J} u=1, Q_{J}\left(G_{-1 / 2}^{-} u\right)=0 .
$$

As $Q_{J}$ of the right hand side of (91) is 1 . Thus, for the function (91) to be possibly non-zero, we must have

$$
Q_{J} v=1
$$

But then we have

$$
w(v) \geq \frac{1}{2} Q_{J} v=\frac{1}{2}
$$

with equality arising if and only if

$$
v \text { is chiral primary of weight } 1 / 2 \text {. }
$$


Remark 1: We see therefore that in the $N=2$ SUSY case, there is in fact no need to assume that the weight of $U^{k}$ is $>(1 / 2,1 / 2)$ for $k<h$. If the obstruction vanishes for $k<h$, then we have

$$
u^{k}=\frac{1}{k !} \int_{D}\left(G_{-1 / 2}^{-} \tilde{G}_{-1 / 2}^{-} u\right)\left(z_{k}\right) \ldots\left(G_{-1 / 2}^{-} \tilde{G}_{-1 / 2}^{-} u\right)\left(z_{1}\right) u d z_{1} \ldots d z_{k} d \bar{z}_{1} \ldots d \bar{z}_{k}
$$

where the integrand is understood as a $(k+1)$-point function (and not its power series expansion in any particular range), over the unit disk.

Additionally, for any worldsheet $\Sigma$,

$$
U_{\Sigma}^{h}=\frac{1}{h !} \int_{\Sigma}\left(G_{-1 / 2}^{-} \tilde{G}_{-1 / 2}^{-} u\right)\left(z_{h}\right) \ldots\left(G_{-1 / 2}^{-} \tilde{G}_{-1 / 2}^{-} u\right)\left(z_{1}\right) d z_{1} \ldots d z_{h} d \bar{z}_{1} \ldots d \bar{z}_{h}
$$

(it is to be understood that in both (94), (95), the fields are inserted into holomorphic images of disks where the origin maps to the point of insertion with derivative of modulus 1 with respect to the measure of integration).

When the obstruction occurs at step $k$, the integral (94) has a divergence of logarithmic type. In the $N=0$ case, there is a third possibility, namely that the obstruction vanishes, but the field $u_{h}$ in Theorem 2 has summands of weight $<(1,1)$ $(<(1 / 2,1 / 2)$ for $N=1)$. In this case, the integral (94) will have a divergence of power type, and the intgral of terms of weight $<(1,1)$ (resp. $<(1 / 2,1 / 2)$ ) has to be taken in range from $\infty$ to 1 rather than from 0 to 1 to get a convergent integral. The formula (95) is not correct in that case.

Remark 2: In [19], a different correlation function is considered as an measure of marginality of $u$ to higher perturbative order. The situation there is actually more general, allowing combinations of both chiral and antichiral primaries. In the present setting of chiral primaries only, the correlation function considered in [19] amounts to

$$
\left\langle 1 \mid\left(G_{-1 / 2}^{-} u\right)\left(z_{n}\right) \ldots\left(G_{-1 / 2}^{-} u\right)\left(z_{1}\right)\right\rangle .
$$

It is easy to see using the standard contour deformation argument to show that (96) indeed vanishes, which is also observed in [18. In [19, this type of vanishing is taken as evidence that the $N=2$ CFT deformations exist. It appears, however, that even though the vanishing of (96) follows from the vanishing of (91), the opposite implication does not hold. (In fact, we will see examples in Section 6 below.) The explanation seems to be that [19] writes down an integral expressing the change of central charge when deforming by a combination of cc fields and ac fields, and proves its vanishing. While this is correct formally, we see from Remark 1 above that in fact a singularity can occur in the integral when our obstruction is non-zero: the integral can marginally diverge for $k$ points while it is convergent for $<k$ points.

It would be nice if the obstruction theory a la Gerstenhaber we described here settled in general the question of deformations of conformal field theory, at least in the vertex operator formulation. It is, however, not that simple. The trouble is that we are not in a purely algebraic situation. Rather, compositions of operators which are infinite series may not converge, and even if they do, the convergence 
cannot be understood in the sense of being eventually constant, but in the sense of analysis, i.e. convergence of sequences of real numbers.

Specifically, in our situation, there is the possibility of divergence of the terms on the right hand side of (42). Above we dealt with one problem, that in general, we do not expect infinitesimal deformations to converge on the degenerate worldsheets of vertex operators, so we may have to replace (42) by equations involving finite annuli instead. However, that is not the only problem. We may encounter regularization along the flow parameter. This stems from the fact that the equations (40), (41) only determine $u(\epsilon)$ up to scalar multiple, where the scalar may be of the form

$$
1+\sum_{i \geq 1} K_{i} \epsilon^{i}=f(\epsilon)
$$

But the point is (as we shall see in an example in the next section) that we may only be able to get a well defined value of

$$
f^{-1}(\epsilon) u(\epsilon)=v(\epsilon)
$$

when the constants $K_{i}$ are infinite. The obstruction then is

$$
\begin{aligned}
& L_{n}(m) f(m) v(m)=0 \in V[\epsilon] / \epsilon^{m+1} \text { for } n>0 \\
& \left(1-L_{0}(m)\right) f(m) v(m)=0 \in V[\epsilon] / \epsilon^{m+1} .
\end{aligned}
$$

At first, it may seem that it is difficult to make this rigorous mathematically with the infinite constants present. However, we may use the followng trick. Suppose we want to solve

$$
\begin{gathered}
c_{1} a_{11}+\ldots+c_{n} a_{1 n}=b_{1} \\
\ldots \\
c_{1} a_{m 1}+\ldots+c_{n} a_{m n}=b_{n}
\end{gathered}
$$

in a, say, finite-dimensional vector space $V$. Then we make rewrite (100) as

$$
\left(b_{1}, \ldots, b_{n}\right)=0 \in\left(\sum_{m} V\right) /\left\langle\left(a_{11}, \ldots, a_{m 1}\right), \ldots,\left(a_{1 n}, \ldots, a_{m n}\right)\right\rangle .
$$

This of course doesn't give anything new in the algebraic situation, i.e when the $a_{i j}$ 's are simply elements of the vector space $V$. When, however the vectors

$$
\left(a_{11}, \ldots, a_{m 1}\right), \ldots,\left(a_{1 n}, \ldots, a_{m n}\right)
$$

are (possibly divergent) infinite sums

$$
\left(a_{1 j}, \ldots, a_{m j}\right)=\sum_{k}\left(a_{1 j k}, \ldots, a_{m j k}\right)
$$

then the right hand side of (101) can be interpreted as

$$
\left(\sum_{m} V\right) /\left\langle\left(a_{11 k}, \ldots, a_{m 1 k}\right), \ldots,\left(a_{1 n k}, \ldots, a_{m n k}\right)\right\rangle .
$$

In that sense, (101) always makes sense, while (100) may not when interpreted directly. We interpret (99) in this way. 
Let us now turn to the question of sufficient conditions for exponentiation of infinitesimal deformations. Suppose there exists a subspace $W \subset V$ closed under vertex operators which contains $u$ and such that for all elements $v \in W$, we have that

$$
\sum_{i} Y_{i}(u, z) \tilde{Y}_{i}(u, \bar{z}) v
$$

involve only $z^{n} \bar{z}^{m}$ with $m, n \in \mathbb{Z}, m, n \neq-1$. Then, by Theorem 1 ,

$$
1-\phi \epsilon: W \rightarrow \hat{W}[\epsilon] / \epsilon^{2}
$$

is an infinitesimal isomorphism between $W$ and the infinitesimally $u$-deformed $W$. It follows, in the non-regularized case, that then

$$
\exp (-\phi \epsilon) u
$$

is a globally deformed primary field of weight $(1,1)$, and

$$
\exp (-\phi \epsilon): W \rightarrow \hat{W}[[\epsilon]]
$$

is an isomorphism between $W$ and the exponentiated deformation of $W$. However, since we now know the primary fields along the deformation, vacua can be recovered from the equation (6) of the last section.

Such nonregularized exponentiation occurs in the case of the coset construction. Setting

$$
W=\langle v| \begin{aligned}
& \left.Y_{i}(u, z) \tilde{Y}_{i}(u, \bar{z}) v \text { involve only } z^{n} \bar{z}^{m} \text { with }\right\rangle . \\
& m, n \geq 0, m, n \in \mathbb{Z}
\end{aligned}
$$

Then $W$ is called the coset of $V$ by $u$. Then $W$ is closed under vertex operators, and if $u \in W$, the formulas (102), (103) apply without regularization.

The case with regularization occurs when there exists some constant

$$
K(\epsilon)=1+\sum_{n \geq 1} K_{n} \epsilon^{n}
$$

where $K_{n}$ are possibly constants such that

$$
K(\epsilon) \exp (-\phi \epsilon) u
$$

is finite in the sense described above (see (101)). We will see an example of this in the next section.

All these constructions are easily adapted to supersymmetry. The formulas (102), (103) hold without change, but the deformation is with respect to $G_{-1 / 2} \tilde{G}_{-1 / 2} u$ resp. $G_{-1 / 2}^{-} \tilde{G}_{-1 / 2}^{-} u, G_{-1 / 2}^{+} \tilde{G}_{-1 / 2}^{-} u$ depending on the situation applicable. 


\section{THE DEFORMATIONS OF FREE FIELD THEORIES}

As our first application, let us consider the 1-dimensional bosonic free field conformal field theory, where the deformation field is

$$
u=x_{-1} \tilde{x}_{-1}
$$

In this case, the infinitesimal isomorphism of Theorem 1 satisfies

$$
\phi=\pi \sum_{n \in \mathbb{Z}} \frac{x_{-n} \tilde{x}_{-n}}{n}
$$

and the sufficient condition of exponentiability from the last section is met when we take $W$ the subspace consisting of states of momentum 0 . Then $W$ is closed under vertex operators, $u \in W$ and the $n=0$ term of (106) drops out in this case. However, this is an example where regularization is needed. It can be realized as follows: Write

$$
\phi=\sum_{n>0} \phi_{n}
$$

where

$$
\phi_{n}=\pi\left(\frac{x_{-n} \tilde{x}_{-n}}{n}-\frac{x_{n} \tilde{x}_{n}}{n}\right) .
$$

We have

$$
\exp \phi=\prod_{n>0} \exp \phi_{n}
$$

To calculate $\exp \phi_{n}$ explicitly, we observe that

$$
\left[\frac{x_{-n} \tilde{x}_{-n}}{n}, \frac{x_{n} \tilde{x}_{n}}{n}\right]=-\frac{x_{-n} x_{n}}{n}-\frac{\tilde{x}_{-n} \tilde{x}_{n}}{n}-1,
$$

and setting

$$
\begin{aligned}
e & =\frac{x_{-n} \tilde{x}_{-n}}{n}, \\
f & =\frac{x_{n} \tilde{x}_{n}}{n}, \\
h & =-\frac{x_{-n} x_{n}}{n}-\frac{\tilde{x}_{-n} \tilde{x}_{n}}{n}-1,
\end{aligned}
$$

we obtain the $s l_{2}$ Lie algebra

$$
\begin{aligned}
& {[e, f]=h,} \\
& {[e, h]=2 e,} \\
& {[f, h]=-2 f .}
\end{aligned}
$$

Note that conventions regarding the normalization of $e, f, h$ vary, but the relations (109) are satisfied for example for

$$
e=\left(\begin{array}{ll}
0 & 1 \\
0 & 0
\end{array}\right), f=\left(\begin{array}{rr}
0 & 0 \\
-1 & 0
\end{array}\right), h=\left(\begin{array}{rr}
-1 & 0 \\
0 & 1
\end{array}\right)
$$


In $S L_{2}$, we compute

$$
\begin{aligned}
& \exp \pi \epsilon(-f+e)=\exp \pi \epsilon\left(\begin{array}{ll}
0 & 1 \\
1 & 0
\end{array}\right) \\
& =\left(\begin{array}{cc}
\cosh \pi \epsilon & \sinh \pi \epsilon \\
\sinh \pi \epsilon & \cosh \pi \epsilon
\end{array}\right) \\
& \left(\begin{array}{cc}
1 & \tanh \pi \epsilon \\
0 & 1
\end{array}\right)\left(\begin{array}{cc}
\frac{1}{\cosh \pi \epsilon} & 0 \\
0 & \cosh \pi \epsilon
\end{array}\right)\left(\begin{array}{cc}
1 & 0 \\
\tanh \pi \epsilon & 1
\end{array}\right) .
\end{aligned}
$$

In the translation (108), this is

$\exp \left(\tanh (\pi \epsilon) \frac{x_{-n} \tilde{x}_{-n}}{n}\right) \exp \left((-\ln \cosh \pi \epsilon)\left(\frac{x_{-n} x_{n}}{n}+\frac{\tilde{x}_{-n} \tilde{x}_{n}}{n}+1\right)\right) \exp \left(-\tanh (\pi \epsilon) \frac{x_{n} \tilde{x}_{n}}{n}\right)$.

To exponentiate the middle term, we claim

$$
\left.\exp \left(\frac{x_{-n} x_{n}}{n} z\right)=: \exp \frac{x_{-n} x_{n}}{n}\left(e^{z}-1\right)\right):
$$

To prove (113), differentiate both sides by $z$. On the left hand side, we get

$$
\frac{x_{-n} x_{n}}{n} \exp \left(\frac{x_{-n} x_{n}}{n} z\right)
$$

Thus, if the derivative by $z$ of the right hand side $y$ of (113) is

$$
\frac{x_{-n} x_{n}}{n}: \exp \left(\frac{x_{-n} x_{n}}{n}\left(e^{z}-1\right)\right):
$$

then we have the differential equation $y^{\prime}=\frac{x_{-n} x_{n}}{n} y$, which proves (113) (looking also at the initial condition at $z=0$ ).

Now we can calculate (114) by moving the $x_{n}$ occuring before the normal order symbol to the right. If we do this simply by changing (114) to normal order, we get

$$
: \frac{x_{-n} x_{n}}{n} \exp \left(\frac{x_{-n} x_{n}}{n}\left(e^{z}-1\right)\right):,
$$

but if we want equality with (114), we must add the terms coming from the commutator relations $\left[x_{n}, x_{-n}\right]=n$, which gives the additional term

$$
\left(e^{z}-1\right): \frac{x_{-n} x_{n}}{n} \exp \left(\frac{x_{-n} x_{n}}{n}\left(e^{z}-1\right)\right): .
$$

Adding together (115) and (116) gives

$$
e^{z}: \frac{x_{-n} x_{n}}{n} \exp \left(\frac{x_{-n} x_{n}}{n}\left(e^{z}-1\right)\right):,
$$

which is the derivative by $z$ of the right hand side of (113), as claimed.

Using (113), (112) becomes

$$
\begin{aligned}
& \Phi_{n}=\frac{1}{\cosh \pi \epsilon} \exp \left(\tanh (\pi \epsilon) \frac{x_{-n} \tilde{x}_{-n}}{x_{n}}\right) \\
& : \exp \left(\left(\frac{1}{\cosh \pi \epsilon}-1\right)\left(\frac{x_{-n} x_{n}}{n}+\frac{\tilde{x}_{-n}^{n} \tilde{x}_{n}}{n}+1\right)\right): \exp \left(-\tanh (\pi \epsilon) \frac{x_{n} \tilde{x}_{n}}{n}\right)
\end{aligned}
$$

which is in normal order. Let us write

$$
\Phi_{n}=\frac{1}{\cosh \pi \epsilon} \Phi_{n}^{\prime} .
$$

Then the product

$$
\Phi^{\prime}=\prod_{n \geq 1} \Phi_{n}^{\prime}
$$


is in normal order, and is the regularized isomorphism from the exponentiated $\epsilon$ deformation $W_{\epsilon}$ of the conformal field theory in vertex operator formulation on to the original $W$. The inverse, which goes from $W$ to $W_{\epsilon}$, is best calculated by regularizing the exponential of $-\phi$. We get

$$
\begin{aligned}
& \exp \pi \epsilon(f-e)=\exp \pi \epsilon\left(\begin{array}{rr}
0 & -1 \\
-1 & 0
\end{array}\right) \\
& =\left(\begin{array}{cc}
\cosh \pi \epsilon & -\sinh \pi \epsilon \\
-\sinh \pi \epsilon & \cosh \pi \epsilon
\end{array}\right) \\
& \left(\begin{array}{cc}
1 & -\tanh \pi \epsilon \\
0 & 1
\end{array}\right)\left(\begin{array}{cc}
\frac{1}{\cosh \pi \epsilon} & 0 \\
0 & \cosh \pi \epsilon
\end{array}\right)\left(\begin{array}{cc}
1 & 0 \\
-\tanh \pi \epsilon & 1
\end{array}\right)= \\
& \frac{1}{\cosh \pi \epsilon} \exp \left(-\tanh (\pi \epsilon) \frac{x_{-n} \tilde{x}_{-n}}{n}\right) \\
& : \exp \left(\left(\frac{1}{\cosh \pi \epsilon}-1\right)\left(\frac{x_{-n} x_{n}}{n}+\frac{\tilde{x}_{-n} \tilde{x}_{n}}{n}+1\right)\right): \exp \left(\tanh (\pi \epsilon) \frac{x_{n} \tilde{x}_{n}}{n}\right) .
\end{aligned}
$$

So expressing this as

$$
\Psi_{n}=\frac{1}{\cosh \pi \epsilon} \Psi_{n}^{\prime}
$$

the product

$$
\Psi^{\prime}=\prod_{n \geq 1} \Psi_{n}^{\prime}
$$

is the regularized iso from $W$ to $W_{\epsilon}$.

Even though $\Psi^{\prime}$ and $\Phi^{\prime}$ are only elements of $\hat{W}$, the element $u(\epsilon)=\Psi^{\prime} u$ is the regularized chiral primary field in $W_{\epsilon}$, and can be used in a regularized version of the equation (6) to calculate the vacua on $V_{\epsilon}$, which will converge on non-degenerate Segal worldsheets.

In this approach, however, the resulting CFT structure on $V_{\epsilon}$ remains opaque, while as it turns out, in the present case it can be identified by another method.

In fact, to answer the question, we must treat precisely the case missing in Theorem 1, namely when the weight 0 part of the vertex operator of the deforming field, which in this case is determined by the momentum, doesn't vanish. The answer is actually known in string theory to correspond to constant deformation of the metric on spacetime, which ends up isomorphic to the original free field theory. From the point of view of string theory, what we shall give is a "purely worldsheet argument" establishing this fact.

Let us look first at the infinitesimal deformation of the operator $Y(v, t, \bar{t})$ for some field $v \in V$ which is an eigenstate of momentum. We have three forms which coincide where defined:

$$
\begin{gathered}
Y\left(x_{-1} \tilde{x}_{1}, z, \bar{z}\right) Y(v, t, \bar{t}) d z d \bar{z} \\
Y(v, t, \bar{t}) Y\left(x_{-1} \tilde{x}_{-1}, z, \bar{z}\right) d z \bar{z} \\
Y\left(Y\left(x_{-1} \tilde{x}_{-1}, z-t, \overline{z-t}\right) v, t, \bar{t}\right) d z d \bar{z} .
\end{gathered}
$$

By chiral splitting, if we assume $v$ is a monomial in the modes, we can denote (121), (122), (123) by $\eta \tilde{\eta}$ (without forming a sum of terms). Again, integrating 
(121)-(123) term by term $d z$, we get forms $\omega_{\infty}, \omega_{0}, \omega_{t}$, respectively. Here we set

$$
\int \frac{1}{z} d z=\ln z
$$

Again, these are branched forms. Selecting points $p_{0}, p_{\infty}, p_{t}$ on the corresponding boundary components, we can, say, make cuts $c_{0, t}$ and $c_{0, \infty}$ connecting the points $p_{0}, p_{t}$ and $p_{0}, p_{\infty}$. Cutting the worldsheet in this way, we obtain well defined branches $\omega_{\infty}, \omega_{0}, \omega_{t}$. To complicate things further, we have constant discrepancies

$$
\begin{aligned}
& C_{0 t}=\omega_{0}-\omega_{t} \\
& C_{0 \infty}=\omega_{0}-\omega_{\infty} .
\end{aligned}
$$

These can be calculated for example by comparing with the 4 point function

$$
Y_{+}\left(x_{-1}, z\right) Y(v, t)+Y(v, t) Y_{-}\left(x_{-1}, z\right)+Y\left(Y_{-}\left(x_{-1}, z-t\right) v, t\right)
$$

where $Y_{-}(v, z)$ denotes the sum of the terms in $Y(v, z)$ involving negative powers of $z$, and $Y_{+}(v, z)$ is the sum of the other terms. Another way to approach this is as follows: one notices that

$$
\int Y\left(x_{-1}, z\right) d z=\left.\partial_{\epsilon} Y\left(1_{\epsilon}, z\right) S_{-\epsilon}\right|_{\epsilon=0}
$$

where $S_{m}$ denotes the operator which adds $m$ to momentum. It follows that

$$
\begin{aligned}
& C_{0 t}=\left.\partial_{\epsilon}\left(Z\left(x_{-1}, v, z, t\right) S_{-\epsilon}-Z\left(x_{-1}, S_{\epsilon} v, z, t\right)\right)\right|_{\epsilon=0} \\
& C_{0 \infty}=\left.\partial_{\epsilon}\left(Z\left(x_{-1}, v, z, t\right) S_{-\epsilon}-S_{\epsilon} Z\left(x_{-1}, v, z, t\right)\right)\right|_{\epsilon=0} .
\end{aligned}
$$

Now the deformation is obtained by integrating the forms

$$
\begin{gathered}
\omega_{0} \tilde{\eta} \\
\left(\omega_{t}+C_{0 t}\right) \tilde{\eta} \\
\left(\omega_{\infty}+C_{0 \infty}\right) \tilde{\eta}
\end{gathered}
$$

on the boundary components around $0, t$ and $\infty$, and along both sides of the cuts $c_{0 t}, c_{0, \infty}$. To get the integrals of the terms in (128)-(130) which do not involve the discrepancy constants, we need to integrate

$$
\left(\sum_{n \neq 0} \frac{x_{-n}}{n} z^{n}+x_{0} \ln z\right)\left(\sum_{m} x_{-m} \bar{z}^{m-1}\right) .
$$

To do this, observe that (pretending we work on the degenerate worldsheet, and hence omitting scaling factors, taking curved integrals over $\|z\|=1$ ),

$$
\begin{gathered}
\oint \frac{\ln z}{\bar{z}} d \bar{z}=-\oint \frac{-\ln \bar{z}}{\bar{z}} d \bar{z}=-2 \pi i \ln \bar{z}-\frac{1}{2}(2 \pi i)^{2} \\
\oint \ln z \cdot \bar{z}^{m-1} d \bar{z}=-2 \pi i \frac{1}{m} \bar{z}^{m} .
\end{gathered}
$$

Integrating (131), we obtain terms

$$
-2 \pi i x_{0}\left(\sum_{m \neq 0} \tilde{x}_{-m} \frac{\bar{z}^{m}}{m}+\tilde{x}_{0} \ln \bar{z}\right)
$$


which will cancel with the integral along the cuts (to calculate the integral over the cuts, pair points on both sides of the cut which project to the same point in the original worldsheet), and "local" terms

$$
\frac{2 \pi i}{2} \sum_{n \neq 0} \frac{x_{-n} \tilde{x}_{-n}}{n}-\frac{1}{2}(2 \pi i)^{2} x_{0} \tilde{x}_{0} .
$$

The discrepancies play no role on the cuts (as the forms $C_{0 t} \tilde{\eta}, C_{0, \infty} \tilde{\eta}$ are unbranched), but using the formula (127), we can compensate for the discrepancies to linear order in $\epsilon$ by applying on each boundary component

$$
S_{-2 \pi i \epsilon \tilde{x}_{0}} .
$$

In (134), however, when integrating $\tilde{\eta}$, we obtain also discrepancy terms conjugate to (136), so the correct expression is

$$
S_{-2 \pi i \epsilon \tilde{x}_{0}} \tilde{S}_{-2 \pi i \epsilon x_{0}} .
$$

The term (137) is also "local" on the boundary components, so the sum of (135) and (137) is the formula for the infinitesimal iso between the free CFT and the infinitesimally deformed theory. To exponentiate, suppose now we are working in a $D$-dimensional free CFT, and the deformation field is

$$
M x_{-1} \tilde{x}_{-1} .
$$

Then the formula for the exponentiated isomorphism multiplies left momentum by

$$
\exp \epsilon M
$$

and right momentum by

$$
\exp \epsilon M^{T} \text {. }
$$

But of course, in the free theory, the left momentum must equal to the right momentum, so this formula works only when $M$ is a symmetric matrix. Thus, to cover the general case, we must discuss the case when $M$ is antisymmetric. In this case, it may seem that we obtain indeed a different CFT which is defined in the same way as the free CFT with the exception that the left momentum $m_{L}$ and right momentum $m_{R}$ are related by the formula

$$
m_{L}=A m_{R}
$$

for some fixed orthogonal matrix $A$. As it turns out, however, this theory is still isomorphic to the free CFT. The isomorphism replaces the left moving oscillators $x_{i,-n}$ by their transform via the matrix $A$ (which acts on this Heisenberg representation by transport of structure).

Next, let us discuss the case of deforming gravitaitonal field of non-zero momentum, i.e. when

$$
u=M x_{-1} \tilde{x}_{-1} 1_{\lambda}
$$

with $\lambda \neq 0$. Of course, in order for (141) to be of weight $(1,1)$, we must have

$$
\|\lambda\|=0 \text {. }
$$


Clearly, then, the metric cannot be Euclidean, hence there will be ghosts and a part of our theory doesn't apply. Note that in order for (141) to be primary, we also must have

$$
M=\sum_{i} \mu_{i} \otimes \tilde{\mu}_{i}
$$

where

$$
\left\langle\mu_{i}, \lambda\right\rangle=\left\langle\tilde{\mu}_{i}, \lambda\right\rangle=0 .
$$

Despite the indefinite signature, we still have the primary obstruction, which is (145)

$\operatorname{coeff} f_{z^{-1} \tilde{z}^{-1}}: \sum_{m, n} M x_{-m} \tilde{x}_{-n} z^{m-1} \bar{z}^{n-1} \exp \lambda\left(\sum_{k \neq 0}\left(\frac{x_{-k}}{k} z^{k}+\frac{\tilde{x}_{-k}}{k} \bar{z}^{k}\right)\right): M x_{-1} \tilde{x}_{-1} 1_{\lambda}$

(we omit the $z^{\left\langle\lambda, x_{0}\right\rangle}$ term, since the power is 0 by (142)). In the notation (143), this is

$$
\begin{aligned}
& \sum_{i, j}\left(\mu_{i} x_{0}-\mu_{i} x_{0} \lambda x_{-1} \lambda x_{1}+\mu_{i} x_{-1} \lambda x_{1}+\lambda x_{-1} \mu_{i} x_{1}\right) \otimes \\
& \left(\tilde{\mu}_{j} \tilde{x}_{0}-\tilde{\mu}_{j} \tilde{x}_{0} \lambda \tilde{x}_{-1} \lambda \tilde{x}_{1}+\tilde{\mu}_{j} \tilde{x}_{-1} \lambda \tilde{x}_{1}+\lambda \tilde{x}_{-1} \tilde{\mu}_{j} \tilde{x}_{1}\right) M x_{-1} \tilde{x}_{-1} 1_{\lambda}
\end{aligned}
$$

which in the presence of (144) reduces to the condition

$$
\|M\|^{2} \lambda \otimes \lambda x_{-1} \tilde{x}_{-1}=0
$$

This is false unless

$$
\|M\|^{2}=0
$$

which means that (141) is a null state, along which the deformation is not interesting in the sense of string theory. More generally, the distributional form of (146) is

$$
\int_{\left.\|\lambda\|\right|^{2}=0} \lambda \otimes \lambda\|M(\lambda)\|^{2}=0 .
$$

If we set

$$
f(\lambda)=\delta_{\|\lambda\|^{2}=0}\|M(\lambda)\|^{2}
$$

then the Fourier transform of $f$ will be a function $g$ satisfying

$$
\sum \pm \frac{\partial^{2} g}{\partial \lambda_{i}^{2}}=0
$$

where the sings correspond to the metric, which we assume is diagonal with entries \pm 1 . The Fourier transform of the condition (148) is then

$$
\frac{\partial^{2}}{\partial \lambda_{i} \partial \lambda_{j}} g=0 \text {. }
$$

Assuming a decay condition under which the Fourier transform makes sense, (149) implies $g=0$, hence (147), so in this case also the obstruction is nonzero unless (141) is a null state. 
In this discussion, we restricted our attention to deforming fields of gravitational origin. It is important to note that other choices are possible. As a very basic example, let us look at the 1-dimensional Euclidean model. Then there is a possibility of critical fields of the form

$$
a 1_{\sqrt{2}}+b 1_{-\sqrt{2}} .
$$

This includes the sine-Gordon interaction [66] when $a=b$. (We see hyperbolic rather than trigonometric functions because we are working in Euclidean spacetime rather than in the time coordinate, which is the case usually discussed.) The primary obstruction in this case states that the weight $(0,0)$ descendant of (150) applied to (150) is 0. Since the descendant is

$$
(4 a b) x_{-1},
$$

we obtain the condition $a=0$ or $b=0$. It is interesting to note that in the case of the compactification on a circle, these cases where investigated very successfully by Ginsparg [27, who used the obstruction to competely characterize the component of the moduli space of $c=1$ CFT's originating from the free Euclidean compactified free theory. The result is that only free theories compactify at different radii, and their $\mathbb{Z} / 2$-orbifolds occur.

There are many other possible choices of non-gravitational deformation fields, one for each field in the physical spectrum of the theory. We do not discuss these cases in the present paper.

Let us now look at the $N=1$-supersymmetric free field theory. In this case, as pointed out above, in the NS-NS sector, critical gravitational fields for deformations have weight $(1 / 2,1 / 2)$. We could also consider the NS-R and R-R sectors, where the critical weights are $(1 / 2,0)$ and $(0,0)$, respectively. These deforming fields parametrize soul directions in the space of infinitesimal deformations. The soul parameters $\theta, \tilde{\theta}$ have weights $(1 / 2,0),(0,1 / 2)$, which explains the difference of critical weights in these sectors.

Let us, however, focus on the body of the space of gravitational deformations, i.e. the NS-NS sector. Let us first look at the weight $(1 / 2,1 / 2)$ primary field

$$
M \psi_{-1 / 2} \tilde{\psi}_{-1 / 2} \text {. }
$$

The point is that the infinitesimal deformation is obtained by integrating the insertion operators of

$$
G_{-1 / 2} \tilde{G}_{-1 / 2} M \psi_{-1 / 2} \tilde{\psi}_{-1 / 2}=M x_{-1} \tilde{x}_{-1} .
$$

Therefore, (151) behaves exactly the same was as deformation along the field (138) in the bosonic case. Again, if $M$ is a symmetric matrix, exponentiating the deformation leads to a theory isomorphic via scaling the momenta, while if $M$ is antisymmetric, the isomorphism involves transforming the left moving modes by the orthogonal matrix $\exp (M)$.

In the case of momentum $\lambda \neq 0$, we again have indefinite signature, and the field

$$
u=M \psi_{-1 / 2} \tilde{\psi}_{-1 / 2} 1_{\lambda} .
$$

Once again, for (152) to be primary, we must have (143), (144). Moreover, again the actual infinitesimal deformation is got by applying the insertion operators of 
$G_{-1 / 2} \tilde{G}_{-1 / 2} u$, so the treatment is exactly the same as deformation along the field (141) in the bosonic case. Again, we discover that under a suitable decay condition, the obstruction is always nonzero for gravitational deformations of non-zero momentum with suitable decay conditions.

It is worth noting that in both the bosonic and supersymmetric cases, one can apply the same analysis to free field theories compactified on a torus. In this case, however, scaling momenta changes the geometry of the torus, so using deformation fields of 0 momentum, we find exponential deformations which change (constantly) the metric on the torus. This seems to confirm, in the restricted sense investigated here, a conjecture stated in [56].

Remark: Since one can consider Calabi-Yau manifolds which are tori, one sees that there should also exist an $N=2$-supersymmetric version of the free field theory compactified on a torus. (It is in fact not difficult to construct such model directly, it is a standard construction.) Now since we are in the Calabi-Yau case, marginal $c c$ fields should correspond to deformations of complex structure, and marginal $a c$ fields should correspond to deformations of Kähler metric in this case.

But on the other hand, we already identified gravitational fields which should be the sources of such deformations. Additionally, deformations in those direction require regularization of the deformation parameter, and hence cannot satisfy the conclusion of Theorem 3 .

This is explained by observing that we must be careful with reality. The gravitational fields we considered are in fact real, but neither chiral nor antichiral primary in either the left or the right moving sector. By contrast, chiral primary fields ( or antiprimary) fields are not real. This is due to the fact that $G_{-3 / 2}^{+}$and $G_{-3 / 2}^{-}$ are not real in the $N=2$ superconformal algebra, but are in fact complex conjugate to each other. Therefore, to get to the real gravitational fields, we must take real parts, or in other words linear combinations of chiral and antichiral primaries, resulting in the need for regularization.

It is in fact a fun exercise to calculate explicitly how our higher $N=2$ obstruction theory operates in this case. Let us consider the $N=2$-supersymmetric free field theory, since the compactification behaves analogously. The minimum number of dimensions for $N=2$ supersymmetry is 2 . Let us denote the bosonic fields by $x, y$ and their fermionic superpartners by $\xi, \psi$. Then the 0 -momentum summand of the state space (NS sector) is (a Hilbert completion of)

$$
\operatorname{Sym}\left(x_{n}, y_{n} \mid n<0\right) \otimes \Lambda\left(\xi_{r}, \psi_{r} \mid r<0, r \in \mathbb{Z}+\frac{1}{2}\right) .
$$

The "body" parts of the bosonic and fermionic vertex operators are given by the usual formulas

$$
\begin{gathered}
Y\left(x_{-1}, z\right)=\sum x_{-n} z^{n-1}, \quad Y\left(y_{-1}, z\right)=\sum y_{-n} z^{n-1} \\
Y\left(\xi_{-1 / 2}, z\right)=\sum \xi_{-s} z^{n-s-1 / 2} \quad Y\left(\psi_{-1 / 2}, z\right)=\sum \psi_{-s} z^{n-s-1 / 2}, \\
{\left[\xi_{r}, \xi_{-r}\right]=\left[\psi_{r}, \psi_{-r}\right]=1} \\
{\left[x_{n}, x_{-n}\right]=\left[y_{n}, y_{-n}\right]=n .}
\end{gathered}
$$

We have, say,

$$
\begin{aligned}
G_{-3 / 2}^{1} & =\xi_{-1 / 2} x_{-1}+\psi_{-1 / 2} y_{-1} \\
G_{-3 / 2}^{2} & =\xi_{-1 / 2} y_{1}-\psi_{-1 / 2} x_{-1}
\end{aligned}
$$


As usual,

$$
G_{-3 / 2}^{ \pm}=\frac{1}{\sqrt{2}}\left(G_{-3 / 2}^{1} \pm i G_{-3 / 2}^{2}\right) .
$$

With these conventions, we have a critical chiral primary

$$
u=\xi_{-1 / 2}-i \psi_{-1 / 2}
$$

(and its complex conjugate critical antichiral primary). We then see that for a non-ero coefficient $C$,

$$
C G_{-1 / 2}^{-} u=x_{-1}-i y_{-1} .
$$

We now notice that formulas analogous to (108) etc. apply to (154), but the -1 summans of $h$ will appear with opposite signs for the real and imaginary summands, so it will cancel out, so the regularization (119), (120) are not needed, as expected.

Next, let us study the formula (77). The key observation here is that we have the combinatorial identity

$$
\frac{1}{n_{1} \ldots n_{k}}=\sum_{\sigma} \frac{1}{\left(n_{\sigma(1)}+\ldots n_{\sigma(k)}\right)\left(n_{\sigma(1)}+\ldots n_{\sigma(k-1)}\right) \ldots n_{\sigma(1)}}
$$

where the sum on the right is over all permutations on the set $\{1, \ldots, k\}$. Now in the present case, we have the infinitesimal iso on the 0-momentum part, up to non-zero coefficient,

$$
\phi=\sum \frac{\left(x_{n}-i y_{n}\right)\left(\tilde{x}_{n}+i \tilde{y}_{n}\right)}{n}
$$

and in the absence of regularization, the expansion of the exponentiated isomorphism on the 0 -momentum parts is simply

$$
\exp (\phi \epsilon) \text {. }
$$

(The + sign in the "s is caused by the fact that we are in the complex conjugate Hilbert space.) Applying this to (153), we see that we have formulas analogous to (112)-(118), and applying the exponentiated iso to (153), all the terms in normal order involving $x_{>0}, y_{>0}$ will vanish, so we end up with

$$
\prod_{n<0} \exp \left(D \frac{\left(x_{n}-i y_{n}\right)\left(\tilde{x}_{n}+i \tilde{y}_{n}\right)}{n}\right) u
$$

for some non-zero coefficient $D$. Applying (155), we get (77).

Finally, the obstruction in chiral form

$$
\left\langle u^{*}(0),\left(G_{-1 / 2}^{-} u\right)\left(z_{k}\right), \ldots,\left(G_{-1 / 2}^{-} u\right)\left(z_{1}\right), u(0)\right\rangle
$$

must vanish identically. To see this, we simply observe (153), (154) that in the present case, $u$ is in the coset model with respect to $G_{-1 / 2}^{-} u$ (see the discussion below formula (243) below). Thus, in the $N=2$-free field theory, the obstruction theory works as expected, and in the case discussed, the obstructions vanish. It is worth noting that in $2 n$-dimensional $N=2$-free field theory, we thus have an $n^{2}$ dimensional space of $c c+a a$ real fields, and an $n^{2}$-dimensional space of real $c a+a c$ fields, and although regularization occurs, there is no obstruction to exponentiating the deformation by turning on any linear combination of those fields. For a free $N=$ 2 -theory compactified on an $n$-dimensional abelian variety, this precisely recovers 
the deformations in the corresponding component of the moduli space of Calabi-Yau varieties.

However, other deformations exist. For an interesting calculation of deformations of the $N=2$-free field theory in "sine-Gordon" directions, see [13].

\section{The Gepner model of the Fermat quintic}

The finite weight states of one chirality (say, left moving) of the Gepner model of the Fermat quintic are embedded in the 5 -fold tensor product of the $N=2$ supersymmetric minimal model of central charge 9/5 [24, 25, 29]. More precisely, the Gepner model is an orbifold construction. This construction has two versions. In 24, 25, 29, one is interested in actual string theories, so the 5 -fold tensor product of central charge 9 of $N=2$ minimal models is tensored with a free supersymmetric CFT on 4 Minkowski coordinates. This is then viewed in lightcone gauge, so in effect, one tensors with a 2-dimensional supersymmetric Euclidean free CFT, resulting in $N=2$-supersymmetric CFT of central charge 12 . Finally, one performs an orbifolding/GSO projection to give a candidate for a theory for which both modularity and spacetime SUSY can be verified.

It is also possible to create an orbifold theory of central charge 9 which is the candidate of the non-linear $\sigma$-model itself, without the spacetime coordinates. (The spacetime coordinates can be added to this construction and usual GSO projection performed if one is interested in the corresponding string theory.)

The essence of this construction not involving the spacetime coordinates is formula (2.10) of [30. In the case of the level $3 N=2$-minimal model, the orbifold construction is with respect to the $\mathbb{Z} / 5$-action diagonal which acts on the eigenstates of $J_{0}$-eigenvalue (="U(1)-charge") $j / 5$ by $e^{2 \pi i j / 5}$. As we shall review, the NS part of the level $3 N=2$ minimal model has two sectors of $U(1)$-charge $j / 5$, which we will for the moment ad hoc denote $H_{j / 5}$ and $H_{j / 5}^{\prime}$ for $j \in \mathbb{Z} / 5 \mathbb{Z}$. In the FF realization (see below), these sectors correspond to $\ell=0, \ell=1$, respectively. Then the NS-NS sector of the 5 -fold tensor product of minimal model has the form

$$
\sum_{\left(i_{k}\right)} \hat{\bigotimes}_{k=1}^{5}\left(H_{i_{k} / 5} \hat{\otimes} H_{i_{k} / 5}^{*} \oplus H_{i_{k} / 5}^{\prime} \hat{\otimes} H_{i_{k} / 5}^{\prime *}\right)
$$

The corresponding sector the orbifold construction (formula (2.10) of [30]) of the orbifold construction has the form

$$
\sum_{\left(i_{k}\right): \sum i_{k} \in 5 \mathbb{Z}} \sum_{j \in \mathbb{Z} / 5 \mathbb{Z}} \hat{\otimes}_{k=1}^{5}\left(H_{i_{k} / 5} \hat{\otimes} H_{\left(j+i_{k}\right) / 5}^{*} \oplus H_{i_{k} / 5}^{\prime} \hat{\otimes} H_{\left(j+i_{k}\right) / 5}^{\prime *}\right) .
$$

Mathematically speaking, this orbifold can be constructed by noting that, ignoring for the moment supersymmetry, the $N=2$-minimal model is a tensor product of the parafermion theory of the same level and a lattice theory (see 28 and also below). The orbifold construction does not affect the parafermionic factor, and 
on the lattice coordinate, which in this case does not possess a non-zero $\mathbb{Z} / 2$ valued form, and hence physically models a free theory compactified on a torus, the orbifold simply means replacing the torus by its factor by the free action of the diagonal $\mathbb{Z} / 5$ translation group, which is represented by another lattice theory. On this construction, $N=2$ supersymmetry is then easily restored using the same formulas as in (157), since the $U(1)$-charge of the $G$ 's is integral.

The calculations in this and the next Section proceed entirely in the orbifold (158), and hence can be derived from the structure of the level $3 N=2$-minimal model. It should be pointed out that a mathematical approach to the fusion rules of the $N=2$ minimal models was given in [39]. We shall use the Coulomb gas realization of the $N=2$-minimal model, cf. [31], 50]. Let us restrict attention to the NS sector. Then, essentially, the left moving sector of the minimal model is a subquotient of the lattice theory where the lattice is 3-dimensional, and spanned by

$$
\left(\frac{3}{\sqrt{15}}, 0,0\right),\left(\frac{1}{\sqrt{15}}, \frac{i}{2} \sqrt{\frac{2}{5}}, \frac{i}{2} \sqrt{\frac{2}{3}}\right),\left(\frac{2}{\sqrt{15}}, 0, i \sqrt{\frac{2}{3}}\right) .
$$

We will adopt the convention that we shall abbreviate

$$
(k, \ell, m)_{M M}=(k, \ell, m)
$$

for the lattice label

We shall also write

$$
\left(\frac{k}{\sqrt{15}}, \frac{\ell i}{2} \sqrt{\frac{2}{5}}, \frac{m i}{2} \sqrt{\frac{2}{3}}\right) .
$$

$$
(\ell, m)_{M M}=(m, \ell, m)_{M M} .
$$

Call the oscillator corresponding to the $j$ 'th coordinate $x_{j, m}, j=0,1,2$. Then the conformal vector is

$$
\frac{1}{2} x_{0,-1}^{2}-\frac{1}{2} x_{1,-1}^{2}+\frac{i}{2} \sqrt{\frac{2}{5}} x_{1,-2}+\frac{1}{2} x_{2,-1}^{2} .
$$

The superconformal algebra is generated by

$$
\begin{aligned}
& G_{-3 / 2}^{+}=i \sqrt{\frac{1}{2}}\left(x_{2,-1}-\sqrt{\frac{5}{3}} x_{1,-1}\right) 1_{\left(\frac{5}{\sqrt{15}}, 0, i \sqrt{\frac{2}{3}}\right)} \\
& G_{-3 / 2}^{-}=-i \sqrt{\frac{1}{2}}\left(x_{2,-1}+\sqrt{\frac{5}{3}} x_{1,-1}\right) 1_{\left(-\frac{5}{\sqrt{15}}, 0,-i \sqrt{\frac{2}{3}}\right.} .
\end{aligned}
$$

For future reference, we will sometimes use the notation

$$
(a, b, c) x_{n}=a x_{0, n}+b x_{1, n}+c x_{2, n}
$$

and also sometimes abbreviate

$$
(b, c) x_{n}=(0, b, c) x_{n} .
$$

The module labels are realized by labels

$$
\begin{gathered}
(\ell, m)=1_{\left(\frac{m}{\sqrt{15}}, \frac{i \ell}{2} \sqrt{\frac{2}{3}}, \frac{i m}{2} \sqrt{\frac{2}{3}}\right)}, \\
0 \leq \ell \leq 3, m=-\ell,-\ell+2, \ldots, \ell-2, \ell .
\end{gathered}
$$

It is obvious that to stay within the range (162), we must understand the fusion rules and how they are applied. The basic principle is that labels are indentified as follows: No identifications are imposed on the 0'th lattice coordinate. This means that upon any identification, the 0'th coordinate must be the same for the labels 
identified. Therefore, the identification is governed by the 1st and 2nd coordinates, which give the Coulomb gas (=Feigin-Fuchs) realization of the corresponding parafermionic theory (the "3 state Potts model"). The key point here are the parafermionic currents

$$
\begin{aligned}
& \psi_{1,-2 / 3}=i \sqrt{\frac{1}{2}}\left(x_{2,-1}-x_{1,-1} \sqrt{\frac{5}{3}}\right) 1_{\left(0, i \sqrt{\frac{2}{3}}\right)_{P F}} \\
& \psi_{1,-2 / 3}^{+}=-i \sqrt{\frac{1}{2}}\left(x_{2,-1}+x_{1,-1} \sqrt{\frac{5}{3}}\right) 1_{\left(0,-i \sqrt{\frac{2}{3}}\right)_{P F}}
\end{aligned}
$$

(the 0'the coordinate is omitted). Clearly, the parafermionic currents act on the labels by

$$
\begin{aligned}
& \psi_{1,-2 / 3}:(\ell, m)_{P F} \mapsto(\ell, m+2)_{P F} \\
& \psi_{1,-2 / 3}^{+}:(\ell, m)_{P F} \mapsto(\ell, m-2)_{P F} .
\end{aligned}
$$

The lattice labels $(\ell, m)_{P F}$ allowed are those which have non-negative weight. This condition coincides with (162). Now we impose the identification for parafermionic labels:

$$
(\ell, m)_{P F}=(3-\ell, m-3)_{P F} .
$$

This implies

$$
\begin{aligned}
& (1,-1)_{P F} \sim(2,2)_{P F} \\
& (1,1)_{P F} \sim(2,-2)_{P F} \\
& (0,0)_{P F} \sim(3,-3)_{P F} \sim(3,3)_{P F} .
\end{aligned}
$$

Now in the Gepner model corresponding to the quintic, the (cc)-fields allowed are

$$
\begin{aligned}
& \left((3,2,0,0,0)_{L},(3,2,0,0,0)_{R}\right), \\
& \left((3,1,1,0,0)_{L},(3,1,1,0,0)_{R}\right), \\
& \left((2,2,1,0,0)_{L},(2,2,1,0,0)_{R}\right), \\
& \left((2,1,1,1,0)_{L},(2,1,1,1,0)_{R}\right), \\
& \left((1,1,1,1,1)_{L},(1,1,1,1,1)_{R}\right),
\end{aligned}
$$

and the $(a c)$-field allowed is

$$
\left((-1,-1,-1,-1,-1)_{L},(1,1,1,1,1)_{R}\right) .
$$

Here we wrote $\ell$ for $1_{(\ell, \ell)_{M M}},(\ell=0, \ldots, 3)$, which is a chiral primary in the $N=2$ minimal model of weight $\ell / 10$, and $-\ell$ for $1_{(\ell,-\ell)_{M M}}$, which is antichiral primary of weight $\ell / 10$. The tuple notation in (166)-(171) really means tensor product. We omit permutations of the fields (166)-(169), so counting all permutations, there are 101 fields (166)-(170).

We will need an understanding of the fusion rules in the level 3 Potts model and $N=2$-supersymmetric minimal model of central charge $9 / 5$. In the level 3 Potts model, we have 6 labels

$$
\begin{aligned}
& (0,0)_{P F},(3,1)_{P F},(3,-1)_{P F}, \\
& (1,1)_{P F},(1,-1)_{P F},(2,0)_{P F} .
\end{aligned}
$$


This can be described as follows: the labels (172) have the same fusion rules as the lattice $L=\langle i \sqrt{6}\rangle \subset \mathbb{C}$, i.e.

$$
L^{\prime} / L
$$

where $L^{\prime}$ is the dual lattice (into which $L$ is embedded using the standard quadratic form on $\mathbb{C}$ ). This dual lattice is $\left\langle\frac{i}{2} \sqrt{\frac{2}{3}}\right\rangle$, and the fusion rule is "abelian", which means that the product of labels has only one possible label as outcome, and is described by the product in $L^{\prime} / L$. The label $\pm \frac{i}{2} \sqrt{\frac{2}{3}}$ corresponds to $(0, \pm 2)_{P F} \sim$ $(3, \mp 1)_{P F}$.

Next, the product of $(2,0)_{P F}$ with $(3, \mp 1)_{P F}$ has only one possible outcome, $(2, \pm 2)_{P F}=(1, \mp 1)_{P F}$. The product of $(2,0)_{P F}$ with itself has two possible outcomes, $(2,0)_{P F}$ and $(0,0)_{P F}$. All other products are determined by commutativity, associativity and unitality of fusion rules.

The result can be summarized as follows: We call (172) level 0,3 labels and (173) level 1,2 labels. Every level 1,2 label has a corresponding label of level 0,3. The correspondence is

$$
\begin{array}{ll}
(0,0)_{P F} & \leftrightarrow \\
(3,1)_{P F} & \leftrightarrow(1,0)_{P F} \\
(3,-1)_{P F} & \leftrightarrow(1,1)_{P F}
\end{array}
$$

As described above, the fusion rules on level 0,3 are determined by the lattice theory of $L$. Additionally, multiplication preserves the correspondence (175), while the level of the product is restricted only by requiring that any level added to level 0,3 is the original level.

To put it in another way still, the Verlinde algebra is

$$
\mathbb{Z}[\zeta] /\left(\zeta^{3}-1\right) \otimes \mathbb{Z}[\epsilon] /\left(\epsilon^{2}-\epsilon-1\right)
$$

where $\zeta=(3,1)_{P F}$ and $\epsilon=(2,0)_{P F}$.

In the $N=2$ supersymmetric minimal model (MM) case, we allow labels

$$
(3 k+m, \ell, m)_{M M}
$$

where $(\ell, m)$ is a PF label, $k \in \mathbb{Z}$. Two labels (177) are identified subject to identifications of PF labels, and also

$$
(j, \ell, m)_{M M} \sim(j+15, \ell, m)_{M M},
$$

and, as a result of SUSY,

$$
(j, \ell, m)_{M M} \sim(j-5, \ell, m-2)_{M M} .
$$

(By $\sim$ we mean that the labels (i.e. VA modules) are identified, but we do not imply that the states involved actually coincide; in the case (179), they have different weights.) Recalling again that we abbreviate $(m, \ell, m)_{M M}$ as $(\ell, m)_{M M}$, we get the following labels for the $c=9 / 5 N=2$ SUSY MM:

$$
\begin{aligned}
& (0,0)_{M M} \leftrightarrow(2,0)_{M M} \\
& (3,3)_{M M} \leftrightarrow(2,-2)_{M M} \\
& (3,1)_{M M} \leftrightarrow(1,1)_{M M} \\
& (3,-1)_{M M} \leftrightarrow(1,-1)_{M M} \\
& (3,-3)_{M M} \leftrightarrow(2,2)_{M M} .
\end{aligned}
$$


Again, the left column (180) represents 0, 3 level labels, the right column represents level 1,2 labels. The left column labels multiply as the labels of the lattice superCFT corresponding to the lattice $\Lambda$ in $\mathbb{C} \oplus \mathbb{C}$ spanned by

$$
(\sqrt{15}, 0),\left(\frac{5}{\sqrt{15}}, i \sqrt{\frac{2}{3}}\right)
$$

(recall that a super-CFT can be assigned to a lattice with integral quadratic form; the quadratic form on $\mathbb{C} \oplus \mathbb{C}$ is the standard one, the complexification of the Euclidean inner product). The dual lattice of (181) is spanned by

$$
\left(\frac{3}{\sqrt{15}}, 0\right),\left(\frac{5}{\sqrt{15}}, i \sqrt{\frac{2}{3}}\right)
$$

which correspond to the labels $(3,0,0)_{M M},(5,3,-1)_{M M}$, respectively. We see that

$$
\Lambda^{\prime} / \Lambda \cong \mathbb{Z} / 5 .
$$

In (180), the rows (counted from top to bottom as $0, \ldots, 4)$ match the corresponding residue class (183). The fusion rules for $(2,0)_{M M},(0,0)_{M M}$ are the same as in the $\mathrm{PF}$ case. Hence, again, multiplication of labels preserves the rows (180), and the Verlinde algebra is isomorphic to

$$
\mathbb{Z}[\eta] /\left(\eta^{5}-1\right) \otimes \mathbb{Z}[\epsilon] /\left(\epsilon^{2}-\epsilon-1\right)
$$

where $\eta$ is $(3,3)_{M M}$.

Remark: As remarked in Section 3, the positive definiteness of the modular functor, which is crucial for our theory to work, is a requirement for a physical CFT. It is interesting to note, however, that if we do not include this requirement, other possible choices of real structure are possible on the modular functor: The Verlinde algebra of a lattice modular functor with another modular functor $M$ with two labels 1 and $\epsilon$, and Verlinde algebras (176), (184) are tensor products of lattice Verlinde algebras and the algebra

$$
\mathbb{Z}[\epsilon] /\left(\epsilon^{2}-\epsilon-1\right) .
$$

The real structure of this last modular functor can be changed by multiplying by -1 the complex conjugation in $M_{\Sigma}$ for a worldsheet $\Sigma$ precisely when $\Sigma$ has an odd number of boundary components labelled on level 1, 2. The resulting modular functor of this operation is not positive-definite.

Let us now discuss the question of vertex operators in the PF realization of the minimal model. Clearly, since the 0 'th coordinate acts as a lattice coordinate and is not involved in renaming, it suffices the question for the parafermions. Now in the Feigin-Fuchs realization of the level 3 PF model, any state can be written as

$$
u 1_{\lambda}
$$

where $\lambda$ is one of the labels (162) and $u$ is a state of the Heisenberg representation of the Heisenberg algebra generated by $x_{i, m}, i=1,2, m \neq 0$. The situation is however further complicated by the fact that not all Heisenberg states $u$ are allowed for a given label $\lambda$. We shall call the states which are in the image of the embedding admissible. For example, since the $\lambda=0$ part of the $\mathrm{PF}$ model is isomorphic to the coset model $S U(2) / S^{1}$ of the same level, states

$$
(a, b) x_{-1}(0,0)_{P F}
$$


are not admissible for $(a, b) \neq(0,0)$. One can show that admissible states are exactly those which are generated from the ground states (162) by vertex operators and PF currents. Because not all states are admissible, however, there are also states whose vertex operators are 0 on admissible states. Let us call them null states. For example, since (186) is not admissible, it follows that

$$
(a, b) x_{-1}(3,3)_{P F},
$$

which is easily seen to be admissible for any choice of $(a, b)$, is null.

Determining explicitly which states are admissible and which are null is extremely tricky (cf. 31]). Fortunately, we don't need to address the question for our purposes. This is because we will only deal with states which are explicitly generated by the primary fields, and hence automatically admissible; because of this, we can ignore null states, which do not affect correlation functions of admissible states.

On the other hand, we do need an explicit formula for vertex operators. One method for obtaining vertex operators is as follows. We may rename fields using the identifications (165) and also PF currents: a PF current applied to a renamed field must be equal to the same current applied to the original field. Note that this way we may get Heisenberg states above labels which fail to satisfy (162). Such states are also admissible, even though the corresponding "ground states" (which have the same name as the label) are not. Now if we have two admissible states

$$
u_{i} 1_{\left(\ell_{i}, m_{i}\right)}, i=1,2
$$

where $0 \leq \ell_{i} \leq 3$ and $\ell_{1}+\ell_{2} \leq 3$, then the lattice vertex operator

$$
\left(u_{1} 1_{\left(\ell_{1}, m_{1}\right)}\right)(z) u_{2} 1_{\left(\ell_{2}, m_{2}\right)}
$$

always satisfies our fusion rules, and (up to scalar multiple constant on each module) is a correct vertex operator of the PF theory. This is easily seen simply by the fact that (188) intertwines correctly with module vertex operators (which are also lattice operators).

While in our examples, it will suffice to always consider operators obtained in the form (188), it is important to realize that they do not describe the PF vertex operators completely. The problem is that when we want to iterate vertex operators, we would have to keep renaming states. But when two ground states $1_{\lambda}, 1_{\mu}$ are identified via the formula (165), it does not follow that we would have

$$
u 1_{\lambda}=u 1_{\mu}
$$

for every Heisenberg state $u$. On the contrary, we saw for example that (186) is inadmissible, while (187) is null. One also notes that one has for example the identification

$$
\lambda x_{-1} 1_{\lambda}=L_{-1} 1_{\lambda}=L_{-1} 1_{\mu}=\mu x_{-1} 1_{\mu},
$$

which is not of the form (189).

Because of this, to describe completely the full force of the PF theory, one needs another device for obtaining vertex operators (although we will not need this in the present paper). Briefly, it is shown in [31] that up to scalar multiple, any vertex operator

$$
u(z) v=Y(u, z)(v)
$$


where $u, v$ are admissible states can be written as

$$
\oint \ldots \oint\left(a_{k} x_{-1} 1_{(0,-2)}\right)\left(t_{k}\right) \ldots\left(a_{1} x_{-1} 1_{(0,-2)}\left(t_{1}\right) u(z) v d t_{1} \ldots d t_{k}\right.
$$

where the operators in the argument (191) are lattice vertex operators and the number $k$ is selected to conform with the given fusion rule. While it is easy to show that operators of the form (191) are correct vertex operators on admissible states (again up to scalar multiple constant on each irreducible module), as the "screening operators"

$$
a x_{-1} 1_{(0,-2)}
$$

commute with PF currents, selecting the bounds of integration ("contours") is much more tricky. Despite the notation, it is not correct to imagine these as integrals over closed curves, at least not in general. One approach which works is to bring the argument of (191) to normal order, which expands it as an infinite sum of terms of the form

$$
\prod\left(t_{i}-t_{j}\right)^{\alpha_{i j}} t_{k}^{\beta_{k}}
$$

(where we put $t_{0}=z$ ) with coefficients which are lattice vertex operators. Then to integrate (192), for $\alpha_{i j}, \beta_{k}>0$, we may simply integrate $t_{i}$ from 0 to $t_{i-1}$, and define the integral by analytic continuation in the variables $\alpha_{i j}, \beta_{k}$ otherwise.

The functions obtained in this way are generalized hypergeometric functions, and fail for example the assumptions of Theorem 1 (see Remark 2 after the Theorem). The explanation is in the fact that, as we already saw, the fusion rules are not "abelian" in this case.

\section{The Gepner model: the obstruction}

We will now show that for the Gepner model of the Fermat quintic, the function (91) may not vanish for the deforming field (166). This means, not all perturbative deformations corresponding to marginal fields exist in this case. We emphasize that our result applies to deformations of the CFT itself (of central charge 9). A different approach is possible by embedding the model to string theory, and investigating the deformations in that setting (cf. [16]). Our results do not automatically apply to deformations in that setting.

We will consider

$$
v=u=(3,3,3) \otimes(2,2,2) .
$$

(In the remaining three coordinates, we will always put the vacuum, so we will omit them from our notation.) First note that by Theorem (3), this is actually the only relevant case (91), since the only other chiral primary field of weight $1 / 2$ with only two non-vacuum coordinates is $(2,2,2) \otimes(3,3,3)$, which cannot correlate with the right hand side of (91), whose first coordinate is on level 0,3 . In any case, we will show therefore that the Gepner model has an obstruction against continuous perturbative deformation along the field (166) in the moduli space of exact conformal field theories. 
Now the chiral correlation function (91) is a complicated multivalued function because of the integrals (192), which are generalized hypergeometric functions. As remarked above, the modular functor has a canonical flat connection on the space of degenerate worldsheets whose boundary components are shifts of the unit circle with the identity parametrization. The flat connection comes from the fact that these degenerate worldsheets are related to each other by applying $\exp \left(z L_{-1}\right)$ to their boundary components. This is why we can speak of analytic continuation of a branch of the correlation function corresponding to a particular fusion rule. It can further be shown (although we do not need to use that result here) that the continuations of the correlation function corresponding to any one particular fusion rules generate the whole correlation function (i.e. the whole modular functor is generated by any one non-zero section).

Let us now investigate which number $m$ we need in (91). In our case, we have

$$
G_{-1 / 2}^{-}(u)=G_{-1 / 2}^{-}(3,3,3) \otimes(2,2,2)-(3,3,3) \otimes G_{-1 / 2}^{-}(2,2,2) .
$$

(The sign will be justified later; it is not needed at this point.) The first summand (193) has $x_{0,0}$-charge $(-2 / \sqrt{15}, 2 / \sqrt{15})$, the second has $x_{0,0}$-charge $(3 / \sqrt{15},-3 / \sqrt{15})$. Thus, the charges can add up to 0 only if $m$ is a multiple of 5 . The smallest possible obstruction is therefore for $m=5$, in which case (91) is a 7 point function. Let us focus on this case. This function however is too big to calculate completely. Because of this, we use the following trick.

First, it is equivalent to consider the question of vanishing of the function

$$
\left\langle 1 \mid\left(G_{-1 / 2}^{-1} u\right)\left(z_{5}\right) \ldots\left(G_{-1 / 2}^{-1} u\right)\left(z_{1}\right) u\left(z_{0}\right) \bar{u}(t)\right\rangle .
$$

Now by the OPE, it is possible to transform any correlation function of the form

$$
\langle\ldots \mid \ldots v(z) w(t) \ldots\rangle
$$

to the correlation function

$$
\left\langle\ldots \mid \ldots\left(v_{n} w\right)(t) \ldots\right\rangle
$$

(all other entries are the same). More precisely, (195) is expanded, in a certain range and choice of branch, into a series in $z-t$ with coefficients (196) for values of $n$ belonging to a coset $\mathbb{Q} / \mathbb{Z}$. By the above argument, therefore, the function (195) vanishes if and only if the function (196) vanishes for all possible choices of $n$ associated with one fixed choice of fusion rule.

In the case of (194), we shall divide the fields on the right hand side into two sets $G_{x}, G_{y}$ containing two copies of $G_{-1 / 2}^{-}$each, and a set $G_{z}$ containing the remaining three fields $u, \bar{u}$ and $G_{-1 / 2}^{-}$. Each set $G_{x}, G_{y}, G_{z}$ will be reduced to a single field using the transition from (195) to (196) (twice in the case of $G_{z}$ ). To simplify notation (eliminating the subscripts), we will denote the fields resulting from $G_{x}$, $G_{y}, G_{z}$ by $a(x), b(y), c(z)$, respectively. Thus, $x, y, z$ are appropriate choices among the variables $z_{i}, t$, depending how the transition from (195) to (196) is applied.

This reduces the correlation function (194) to

$$
\langle 1 \mid a(x) b(y) c(z)\rangle .
$$

Most crucially, however, we make the following simplification: We shall choose the fusion rules in such a way that the fields $a, b, c$ are level 0,3 in the Feigin-Fuchs realization, and at most one of the charges will be 3 (in each coordinate). Then, 
(197) is just a lattice correlation function, for the computation of which we have an algorithm.

To make the calculation correctly, we must keep careful track of signs. When taking a tensor product of super-CFT's, one must add appropriate signs analogous to the Koszul-Milnor signs in algebraic topology. Now a modular functor of a superCFT decomposes into an even part and an odd part. Additionally, more than one choice of this decomposition may be possible for the same theory, depending on which bottom states of irreducible modules are chosen as even or odd. The sign of a fusion rule is then determined by whether composition along the pair of pants with given labels preserves parity of states or not. Mathematically, this phenomenon was noticed by Deligne in the case of the determinant line (cf. [47]). (Deligne also noticed that in some cases no consistent choice of signs is possible and a more refined formalism is needed; a single fermion of central charge $1 / 2$ is an example; this is also discussed in [47. However, this will not be needed here.)

In the case of the $N=2$-minimal model, there is a choice of parities of ground states of irreducible modules which make the whole modular functor (all the fusion rules) even: simply choose the parity of $(k, \ell, m)$ to be $k \bmod 2$. We easily see that this is compatible with supersymmetry.

Now in this case of completely even modular functor, the signs simplify, and we put

$$
Y(u \otimes v, z)(r \otimes s)=(-1)^{\pi(u) \pi(v)} Y(u, z) r \otimes Y(v, z) s
$$

(where $\pi(u)$ means the parity of $u$ ). Regarding supersymmetry (if present), an element $H$ of the superconformal algebra also acts on a tensor product by

$$
H(u \otimes v)=H u \otimes v+(-1)^{\pi(H) \pi(u)} u \otimes H v,
$$

in particular

$$
G_{-1 / 2}^{-}(u \otimes v)=\left(G_{-1 / 2}^{-} u\right) \otimes v+(-1)^{\pi(u)} u \otimes\left(G_{-1 / 2}^{-} v\right) .
$$

We see that because of (200), the fields $a, b, c$ may have the form of sum of several terms.

Example 1: Recall that the inner product (more precisely symmetric bilinear form) of labels considered as lattice points is

$$
\left\langle\left(r_{1}, s_{1}, t_{1}\right),\left(r_{2}, s_{2}, t_{2}\right)\right\rangle=\frac{r_{1} r_{2}}{15}+\frac{s_{1} s_{2}}{10}-\frac{t_{1} t_{2}}{6} .
$$

Recall also (from the definition of energy-momentum tensor) that weight of the label ground states is calculated by

$$
w(r, s, t)_{M M}=\frac{r^{2}}{30}+w(s, t)_{P F}=\frac{r^{2}}{30}+\frac{s(s+2)}{20}-\frac{t^{2}}{12} .
$$

Now we have

$$
u=(3,3,3) \otimes(2,2,2)=(3,0,0) \otimes(2,1,-1) .
$$

We begin by choosing the field $c$. Compose first $u$ and

$$
\bar{u}=(-3,3,-3) \otimes(-2,2,-2)=(-3,0,0) \otimes(-2,1,1) .
$$


We choose the non-zero $u_{n} \bar{u}$ of the bottom weight for the fusion rule which adds the lattice charges on the right hand side of (203), (204). The result is

$$
u_{-1 / 10} \bar{u}=(0,0,0) \otimes(0,2,0) .
$$

Next, apply $G_{-1 / 2}^{-} u$ to (205). Again, we will choose the bottom descendant. Now $G_{-1 / 2}^{-} u$ has two summands,

$$
(-2,3,1) \otimes(2,1,-1)
$$

and

$$
(3,0,0) \otimes(0,5,3) x_{-1}(-3,1,3)
$$

(the term (207) involves renaming to stay withing no-ghost PF labels after composition). Applying (206) to (205) gives bottom descendant

$$
(-2,3,1) \otimes(2,3,-1) \text { of weight } 8 / 5,
$$

applying (207) to (205) gives bottom descendant

$$
(3,0,0) \otimes(-3,0,0) \text { of weight } 3 / 5 \text {. }
$$

Since (209) has lower weight than (208), (208) may be ignored, and we can choose

$$
c=(3,0,0) \otimes(-3,0,0) .
$$

Now again, using the formula (200), we see that in the sets of fields $G_{x}, G_{y}$ we need one summand (207) and three summands (206) to get to $x_{00}$-charge 0 . Thus, one of the groups $G_{x}, G_{y}$ will contain two summands of (206) and the other will contain one. We employ the following convention:

We choose $G_{y}$ to contain two summands (206) and $G_{x}$ to contain one summand (206) and one summand (207).

This leads to the following:

We must choose the fields $a$ and $b$ of the same weights and symmetrize the resulting correlation function with respect to $x$ and $y$.

We will choose $b$ first. Again, we will choose the bottom weight (nonzero) descendant of (206) applied to itself renamed as

$$
(0,5,-3) x_{-1}(-2,0,-2) \otimes(2,2,2),
$$

which is

$$
(-4,3,-1) \otimes(4,3,1)
$$

We rename to level 0 , which gives

$$
\begin{aligned}
& b=(0,5,3) x_{-1}(-4,0,2) \otimes(0,5,-3) x_{-1}(4,0,-2), \\
& w(b)=12 / 5 .
\end{aligned}
$$

Then $a$ must have weight $12 / 5$ to satisfy (212). When calculating $a$, however, there is an additional subtlety. This time, we have actually take into account two summands, from applying (206) to (207) and vice versa, i.e. (207) to (206). In both cases, we must rename to get the desired fusion rule. To this end, we may replace (207) by

$$
(3,0,0) \otimes(-3,2,0) .
$$


However, when applying (206) and (216) to each other in opposite order, the renamings then do not correspond, resulting in the possibility of wrong coefficient/sign (since renaming are correct only up to constants which we haven't calculated). To reconcile this, we must use exactly the same renamings step by step, related only by applying PF currents. To this end, we may compare the renaming of applying

$$
(0,5,-3) x_{-1}(-2,0,-2) \otimes(2,2,2)
$$

to

$$
(3,0,0) \otimes \frac{1}{2}(0,5,-3) x_{-1}(-3,1,-3)
$$

(the $\frac{1}{2}$ comes from the PF current $(5,-3) x_{-1}(0,-2)$ which takes $(2,2)$ to $\left.2(2,0)\right)$ and

$$
(3,0,0) \otimes(-3,2,0)
$$

to

$$
(0,5,-3) x_{-1}(-2,0,-2) \otimes(2,1,-1) .
$$

We see that the bottom descendant of applying (217) to (218) is

$$
(0,5,-3) x_{-1}(1,0,-2) \otimes(-1)(-1,3,-1)
$$

while the bottom descendant of applying (219) to (220) is

$$
(0,5,-3) x_{-1}(1,0,-2) \otimes(-1,3,-1) .
$$

The expression (221) is the negative of (222). On the other hand, we see that the bottom descendants of applying (206) to (216) and vice versa are the same. This means that we are allowed to use the names (206) and (216) to each other in either order, but we must take the results with opposite signs.

Now (222) has weight $7 / 5$, so to get weight $12 / 5$, we must take the descendant of applying (206) to (216) and vice versa which is of weight 1 higher than the bottom. This gives

$$
\begin{aligned}
& ((-2,3,1)-(3,0,0)) x_{-1}(1,3,1) \otimes(-1,3,-1)+ \\
& (1,3,1) \otimes((2,1,-1)-(-3,2,0)) x_{-1}(-1,3,-1),
\end{aligned}
$$

which is

$(223) a=(-5,3,1) x_{-1}(1,3,1) \otimes(-1,3,-1)+(1,3,1) \otimes(5,-1,-1) x_{-1}(-1,3,-1)$.

Now the correlation function of $a(x), b(y), c(z)$ given in (223), (215), (210) is an ordinary lattice correlation function. The algorithm for calculating the lattice correlation function of fields $u_{i}\left(x_{i}\right)$ which are of the form

$$
1_{\lambda_{i}}\left(x_{i}\right)
$$

or

with the label

$$
\mu_{i} x_{-1} 1_{\lambda_{i}}\left(x_{i}\right)
$$

$$
1_{\sum \lambda_{i}}
$$

is as follows: The correlation function is a multiple of

$$
\prod_{i<j}\left(x_{i}-x_{j}\right)^{\left\langle\lambda_{i}, \lambda_{j}\right\rangle}
$$


by a certain factor, which is a sum over all the ways we may "absorb" any $\mu_{i} x_{-1}$ factors. Each such factor may either be absorbed by another $\mu_{j} x_{-1}$, which results in a factor

$$
\left\langle\mu_{i}, \mu_{j}\right\rangle\left(x_{i}-x_{j}\right)^{-2}, i \neq j
$$

or by another lattice label $1_{\lambda_{j}}$, which results in a factor

$$
\left\langle\mu_{i}, \lambda_{j}\right\rangle\left(x_{i}-x_{j}\right)^{-1}, i \neq j .
$$

Each $\mu_{i} x_{-1}$ must be absorbed exactly once (and the mechanism (224) is considered as absorbing both $\mu_{i}$ and $\mu_{j}$ ), but one lattice label $1_{\lambda_{j}}$ may absorb several different $\mu_{i} x_{-1}$ 's via (225).

Evaluating the correlation function of $a(x), b(y), c(z)$ with the vacuum using this algorithm, we get

$$
\frac{2(y-z)}{(x-z)(x-y)^{3}} \text {. }
$$

Symmetrizing with respect to $x, y$, we get

$$
\frac{2(x-2 z+y)}{(y-z)(x-z)(x-y)^{2}},
$$

(our total correlation function factor), which is non-zero.

In more detail, we can calculate separately the contributions to the correlation function of the two summands (223). For the first summand, the factor before the $\otimes \operatorname{sign}$ contributes

$$
-\frac{1}{(x-z)(y-x)},
$$

the factor after the $\otimes$ sign contributes

$$
\frac{1}{y-x} \text {. }
$$

Multiplying (226) and (227), we get

$$
-\frac{1}{(x-z)(x-y)^{2}},
$$

and symmetrizing with respect to $x$ and $y$,

$$
-\frac{x-2 z+y}{(x-z)(x-y)^{2}(y-z)},
$$

which is the total contribution of the first summand (223).

For the second summand (223), the factor after $\otimes$ contributes

$$
-\frac{2}{(x-y)^{2}}-\frac{1}{(x-z)(y-x)},
$$

and the factor before the $\otimes$ sign contributes

$$
\frac{1}{y-x} \text {. }
$$

Multiplying, we get

$$
\frac{x-2 z+y}{(x-z)(x-y)^{3}} .
$$

After symmetrizing with respect to $x, y$, we get also (228), so both summands of (223) contribute equally to the correlation function. 
Example 2: In this example, we keep the same $a(x)$ and $b(y)$ as in the previous example, but change $c(z)$. To select $c(z)$, this time we start with $G_{-1 / 2}^{-} u$ represented as

$$
(3,0,0) \otimes(0,5,-3) x_{-1}(-3,1-3)+C(-2,3,1) \otimes(2,1,-1)
$$

( $C$ is a non-zero normalization constant which we do not need to evaluate explicitly), which we apply to $\bar{u}$ represented as

$$
(-3,0,0) \otimes(-2,1,1)
$$

From the two summands (231), we get bottom descendants

$$
(0,0,0) \otimes(-5,2,-2) \text { of weight } 9 / 10
$$

and

$$
(-5,3,1) \otimes(0,2,0) \text { of weight } 19 / 10 .
$$

Therefore, we may ignore (234) and select (233) only. Now applying (233) to $u$ written as

$$
(3,0,0) \otimes(2,1,-1),
$$

we select a descendant of weight 1 above the label

$$
(3,0,0) \otimes(-3,3,-3) .
$$

Recalling from the conjugate of (187) that weight 1 states above the label $(3,-3)_{P F}=$ $(0,0)_{P F}$ must vanish, we get

$$
c=(3,0,0) \otimes(1,0,0) x_{-1}(-3,0,0)
$$

(up to a non-zero multiplicative constant). This gives the correlation function

$$
\frac{(x-2 z+y)^{2}}{5(y-z)^{2}(x-z)^{2}(x-y)^{2}} .
$$

Let us write again in more detail the contributions of the two summands (223). For the first summand, the contribution of the factor before $\otimes$ is again (226) (hasn't changed), and the contribution of the factor after $\otimes$ is

$$
\frac{-y-3 z+4 x}{15(y-z)(x-z)(x-y)} \text {. }
$$

Multiplying, we get

$$
\frac{-y-3 z+4 x}{15(y-z)^{2}(x-z)^{2}(x-y)},
$$

and symmetrizing with respect to $x, y$,

$$
-\frac{y^{2}+6 y z-6 z^{2}-8 x y+6 z x+x^{2}}{15(y-z)^{2}(x-z)^{2}(x-y)^{2}} .
$$

This is the total contribution of the first summand (223).

For the second summand (223), the coordinate before $\otimes$ contributes again (230), and the coordinate after $\otimes$ contributes

$$
\frac{2\left(-y x-3 y z-3 x z+3 z^{2}+2 x^{2}+2 y^{2}\right)}{15(y-z)(x-z)^{2}(x-y)^{2}} .
$$

Multiplying, we get

$$
-\frac{-y x-3 y z-3 x z+3 z^{2}+2 x^{2}+2 y^{2}}{15(y-z)(x-z)^{2}(x-y)^{3}} .
$$


Symmetrizing with respect to $x, y$, we get

$$
\frac{2\left(-y x-3 y z-3 x z+3 z^{2}+2 x^{2}+2 y^{2}\right)}{15(y-z)^{2}(x-z)^{2}(x-y)^{2}}
$$

which is the total contribution of the second summand (223). Adding the contributions (239) and (241) (which are not equal in this case) gives (237).

The remainder of this Section is dedicated to comments on possible perturbative deformations along the fields $\left(1^{5}, 1^{5}\right),\left(-1^{5}, 1^{5}\right)$ (the exponent here denotes repetition of the field in a tensor product, and 1 again stands for $(1,1,1)_{M M}$, etc.). We will present some evidence (although not proof) that the obstruction might vanish in this case. The results we do obtain will prove useful in the next Section. Such conjecture would have a geometric interpretation. In Gepner's conjectured interpretation of the model we are investigating as the $\sigma$-model of the Fermat quintic, the field (171) corresponds to the dilaton. It seems reasonable to conjecture that the dilaton deformation should exist, since the theory should not choose a particular global size of the quintic. Similarly, the field (170) can be explained as the dilaton on the mirror manifold of the quintic, which should correspond to deformations of complex structure of the form

$$
x^{5}+y^{5}+z^{5}+t^{5}+u^{5}+\lambda x y z t u=0 .
$$

Therefore, our analysis predicts that the (body of) the moduli space of $N=2$ supersymmetric CFT's containing the Gepner model is 2-dimensional, and contains $\sigma$-models of the quintics (242), where the metric is any multiple of the metric for which the $\sigma$-model exists (which is unique up to a scalar multiple).

To discuss possible deformations along the fields $\left(1^{5}, 1^{5}\right),\left(-1^{5}, 1^{5}\right)$, let us first review a simpler case, namely the coset construction: In a VOA $V$, we set, for $u \in V$ homogeneous,

$$
\begin{aligned}
& Y(u, z)=\sum_{n \in \mathbb{Z}} u_{-n+w(u)} z^{n}, \\
& Y_{-}(u, z)=\sum_{n<0} u_{-n+w(u)} z^{n}, \\
& Y_{+}(u, z)=Y(u, z)-Y_{-}(u, z) .
\end{aligned}
$$

The coset model of $u$ is

$$
\begin{aligned}
& V_{u}= \\
& \left.\langle v \in V| Y_{-}(u, z) v=0 \text { and } Y_{+}(u, z) \text { involves only integral powers of } z\right\rangle .
\end{aligned}
$$

Then $V_{u}$ is a sub-VOA of $V$. To see this, recall that

$$
Y(u, z) Y(v, t) w=Y_{+}(u, z) Y_{-}(v, t) w+Y_{+}(v, t) Y_{-}(u, z) w+Y\left(Y_{-}(u, z-t) v\right) w .
$$

When $v, w \in V_{u}$, the last two terms of the right hand side of (245) vanish, which proves that

$$
Y(v, t) w \in V_{u}[[t]]\left[t^{-1}\right] .
$$

Now in the case of $N=2$-super-VOA's, let us stick to the NS sector. Then (243) still correctly describes the "body" of a vertex operator. The complete vertex 
operator takes the form

$(246)$

$$
\begin{aligned}
& Y\left(u, z, \theta^{+}, \theta^{-}\right)= \\
& \sum_{n \in \mathbb{Z}} u_{-n+w(u)} z^{n}+u_{-n-1 / 2+w(u)}^{+} z^{n} \theta^{+}+u_{-n-1 / 2+w(u)}^{-} z^{n} \theta^{-}+u_{-n-1+w(u)}^{ \pm} z^{n} \theta^{+} \theta^{-} .
\end{aligned}
$$

We still define $Y_{-}\left(u, z, \theta^{+}, \theta^{-}\right)$to be the sum of terms involving $n<0$, and $Y_{+}\left(u, z, \theta^{+}, \theta^{-}\right)$the sum of the remaining terms. The compatibility relations for an $N=2$-super-VOA are

$$
\begin{aligned}
& D^{+} Y\left(u, z, \theta^{+}, \theta^{-}\right)=Y\left(G_{-1 / 2}^{+} u, z, \theta^{+}, \theta^{-}\right) \\
& D^{-} Y\left(u, z, \theta^{+}, \theta^{-}\right)=Y\left(G_{-1 / 2}^{-} u, z, \theta^{+}, \theta^{-}\right)
\end{aligned}
$$

where

$$
D^{+}=\frac{\partial}{\partial \theta^{+}}+\theta^{+} \frac{\partial}{\partial z}, \quad D^{-}=\frac{\partial}{\partial \theta^{-}}+\theta^{-} \frac{\partial}{\partial z}
$$

Now using (245) again, for $u \in V$ homogeneous, we will have a sub- $N=2$-VOA $V_{u}$ defined by (244), which is further endowed with the operators $G_{-1 / 2}^{-}, G_{-1 / 2}^{+}$.

In the case of lack of locality, only a weaker conclusion holds. Assume first we have "abelian" fusion rules in the same sense as in Remark 2 after Theorem 1 .

Lemma 4. Suppose we have fields $u_{i}, i=0, \ldots, n$ such that for $i>j$,

$$
Y\left(u_{i}, z\right) u_{j}=\sum_{n \geq 0}\left(u_{i}\right)_{-n-\alpha_{i j}-w\left(u_{i}\right)} z^{n+\alpha_{i j}}
$$

with $0 \leq \alpha_{i j}<1$. Consider further points $z_{0}=0, z_{1}, \ldots, z_{n}$. Then

$$
\prod_{n \geq i>j \geq 0}\left(z_{i}-z_{j}\right)^{-\alpha_{i j}} Y\left(u_{n}, z_{n}\right) \ldots Y\left(u_{z}, z_{1}\right) u_{0}
$$

where each $\left(z_{i}-z_{j}\right)^{-\alpha_{i j}}$ are expanded in $z_{j}$ is a power series whose coefficients involve nonnegative integral powers of $z_{0}, \ldots, z_{n}$ only.

Proof: Induction on $n$. Assuming the statement is true for $n-1$, note that by assumption, (250), when coupled to $w^{\prime} \in V^{\vee}$ of finite weight, is a meromorphic function in $z_{n}$ with possible singularities at $z_{0}=0, z_{1}, \ldots, z_{n}-1$. Thus, (250) can 
be expanded at its singularities, and is equal to (251)

$$
\begin{aligned}
& \prod_{n-1 \geq i>j \geq 0}\left(z_{i}-z_{j}\right)^{-\alpha_{i j}} \\
& \left(\left(z_{n}^{-\alpha_{n 0}} \operatorname{expand}_{z_{n}} \prod_{j \neq 0}\left(z_{n}-z_{j}\right)^{-\alpha_{n j}} Y\left(u_{n-1}, z_{n-1}\right) \ldots Y\left(u_{1}, z_{1}\right) Y\left(u_{n}, z_{n}\right) u_{0}\right)_{z_{n}^{<0}}\right. \\
& +\left(\sum_{i=1}^{n-1}\left(z_{n}-z_{i}\right)^{-\alpha_{n i}} \operatorname{expand}_{\left(z_{n}-z_{i}\right)}\left(\prod_{n-1 \geq j \neq i}\left(z_{n}-z_{j}\right)^{-\alpha_{n j}}\right) .\right. \\
& Y\left(u_{n-1}, z_{n-1}\right) \ldots Y\left(u_{i+1}, z_{i+1}\right) Y\left(Y\left(u_{n}, z_{n}-z_{i}\right) u_{i}, z_{i}\right) \text {. } \\
& \left.Y\left(u_{i-1}, z_{i-1}\right) \ldots Y\left(u_{1}, z_{1}\right) u_{0}\right)_{\left(z_{n}-z_{i}\right)<0} \\
& +\left(z_{n}^{-\alpha_{n 0}-\ldots-\alpha_{n, n-1}} \operatorname{expand}_{1 / z_{n}} \prod_{j=1}^{n-1}\left(1-\frac{z_{j}}{z_{n}}\right)^{-\alpha_{n j}} .\right. \\
& \left.\left.Y\left(u_{n}, z_{n}\right) Y\left(u_{n-1}, z_{n-1}\right) \ldots Y\left(u_{1}, z_{1}\right) u_{0}\right)_{z_{n}^{20}}\right) \text {. }
\end{aligned}
$$

In (251), expand?(?) means that the argument is expanded in the variable given as the subscript. The symbol $(?)_{?<0}$ (resp. $\left.(?)_{? \geq 0}\right)$ means that we take only terms in the argument, (which is a power series in the subscript), which involve negative (resp. non-negative) powers of the subscript.

In any case, by the assumption of the Lemma, all summands (251) vanish with the exception of the last, which is the induction step.

In the case of non-abelian fusion rules, an analogous result unfortunately fails. Assume for simplicity that

$$
\begin{aligned}
& u_{0}=\ldots=u_{n} \text { holds in (251) with } 0 \leq \alpha_{i j}^{F}<1 \text { true for any } \\
& \text { fusion rule } F \text {. }
\end{aligned}
$$

We would like to conclude that the correlation function

$$
\left\langle v, u\left(z_{n}\right) \ldots u\left(z_{1}\right) u\right\rangle
$$

involves only non-negative powes of $z_{i}$ when expanded in $z_{1}, \ldots, z_{n}$ (in this order). Unfortunately, this is not necessarily the case. Note that we know that (253) converges to 0 when two of the argument $z_{i}$ approach while the others remain separate. However, this does not imply that the function (253) converges to 0 when three or more of the arguments approach simultaneously.

To give an example, let us consider the solution of the Fuchsian differential equation of $\mathbb{P}^{1}-\{0, t, \infty\}$

$$
y^{\prime}=\left(\frac{A}{x}+\frac{B}{z-t}\right) y
$$

for square matrices $A, B$ (with $t \neq 0$ constant). Since the solution $y$ has bounded singularities, multiplying $y$ by $z^{m}(z-t)^{n}$ for large enough integers $m, n$ makes the resulting function $Y$ converge to 0 when $z$ approaches 0 or $t$. If, however, the expansion of $Y$ at $\infty$ involved only non-negative powers of $z$, it would have only finitely many terms, and hence abelian monodromy. It is well known, however, that this is not necessarily the case. In fact, any irreducible monodromy occurs for a solution of the equation (254) for suitable matrices $A, B$ (cf. 7]). 
Therefore, the following result may be used as evidence, but not proof, of the exponentiability of deformations along $\left(1^{5}, 1^{5}\right)$ and $\left(1^{5},-1^{5}\right)$.

Lemma 5. The assumption (252) is satisfied for the field

$$
u=G_{-1 / 2}^{-}((1,1,1), \ldots,(1,1,1))
$$

in the 5 -fold tensor product of the $N=2$ minimal model of central charge $9 / 5$.

Before proving this, let us state the following consequence:

Indeed, assuming Lemma 5 and setting $w=((1,1,1), \ldots,(1,1,1))$, the obstruction is

$$
\left\langle w^{\prime} \mid\left(G_{-1 / 2}^{-}\right) w\left(z_{n}\right) \ldots\left(G_{-1 / 2}^{-} w\right)\left(z_{1}\right) w\right\rangle .
$$

(The antichiral primary case is analogous.) But using the fact that

$$
G_{-1 / 2}^{-}\left(\left(G_{-1 / 2}^{-}\right) w\left(z_{n}\right) \ldots\left(G_{-1 / 2}^{-} w\right)\left(z_{1}\right) w\right)=\left(G_{-1 / 2}^{-}\right) w\left(z_{n}\right) \ldots\left(G_{-1 / 2}^{-} w\right)\left(z_{1}\right) G_{-1 / 2}^{-} w
$$

along with injectivity of $G_{-1 / 2}^{-}$on chiral primaries of weight $1 / 2$, we see that the non-vanishing of (255) implies the non-vanishing of (253) with $u=G_{-1 / 2}^{-} w$ for some $v$ of weight 1 , which would contradict Lemma (5).

Proof of Lemma 5: We have

$$
G_{-1 / 2}^{-}(1,1,1)=(-4,1,-1) .
$$

We have in our lattice

$$
\begin{aligned}
& (1,1,1) \cdot(1,1,1)=1 / 15+1 / 10-1 / 6=0, \\
& (-4,1,-1) \cdot(-4,1,-1)=16 / 15+1 / 10-1 / 6=1, \\
& (1,1,1) \cdot(-4,1,-1)=-4 / 15+1 / 10+1 / 6=0,
\end{aligned}
$$

so we see that with the fusion rules which stays on levels 1,2 , the vertex operators $u(z) u$ have only non-singular terms.

However, this is not sufficient to verify (252). In effect, when we use the fusion rule which goes to levels 0,3 ,

$$
(1,1,1)(z)(-4,1,-1)
$$

and

$$
(-4,1,-1)(z)(1,1,1)
$$

will have most singular term $z^{-2 / 5}$, so when we write again 1 instead of $(1,1,1)$ and $G$ instead of $G_{-1 / 2}^{-}(1,1,1)$, with the least favorable choice of fusion rules, it seems $u(z) u$ can have singular term $z^{-4 / 5}$, coming from the expressions

$$
(G 1111)(z)(1 G 111)
$$

and

$$
(1 G 111)(z)(G 1111)
$$

(and expression obtained by permuting coordinates). Note that with other combinations of fusion rules, various other singular terms can arise with $z^{>-4 / 5}$.

Now the point is, however, that we will show that with any choice of fusion rule, the most singular terms of (258) and (259) come with opposite signs and hence cancel out. Since the $z$ exponents of other terms are higher by an integer, this is all we need. 
Recalling the Koszul-Milnor sign rules for the minimal model, recall that 1 is odd and $G$ is even, so

$$
\begin{gathered}
(G \otimes 1)(z)(1 \otimes G)=-G(z) 1 \otimes 1(z) G, \\
(1 \otimes G)(z)(G \otimes 1)=1(z) G \otimes G(z) 1 .
\end{gathered}
$$

We have

$$
\begin{aligned}
& 1(z) G=(1,1,1)(z)(-4,2,2)=M(-3,0,0) z^{-2 / 5}+\mathrm{HOT}, \\
& G(Z) 1=(-4,2,2)(z)(1,1,1)=N(-3,0,0) z^{-2 / 5}+\mathrm{HOT},
\end{aligned}
$$

with some non-zero coefficients $M, N$, so the bottom descendants of (260) and (261) are

$$
-M N(-3,0,0) \otimes(-3,0,0)
$$

resp.

$$
M N(-3,0,0) \otimes(-3,0,0),
$$

so they cancel out, as required.

\section{The Case of the Fermat quartic $K 3$-Surface}

The Gepner model of the $K 3$ Fermat quartic is an orbifold analogous to (158) with 5 replaced by 4 of the 4 -fold tensor product of the level $2 N=2$-minimal model, although one must be careful about certain subtlteties arising from the fact that the level is even. The model has central charge 6 . The level $2 \mathrm{PF}$ model is the 1-dimensional fermion (of central charge 1/2), viewed as a bosonic CFT. As such, that model has 3 labels, the NS label with integral weights (denote by $N S$ ), the NS label with weights $\mathbb{Z}+\frac{1}{2}$ (denote by $N S^{\prime}$ ), and the R label (denote by $R$ ). The fusion rules are given by the fact that $N S$ is the unit label,

$$
\begin{aligned}
& N S^{\prime} * N S^{\prime}=N S, \\
& N S^{\prime} * R=R, \\
& R * R=N S+N S^{\prime} .
\end{aligned}
$$

We shall again find it useful to use the free field realization of the $N=2$ minimal model, which we used in the last two sections. In the present case, the theory is a subquotient of a lattice theory spanned by

$$
\left(\frac{1}{\sqrt{2}}, 0,0\right),\left(\frac{1}{\sqrt{8}}, \frac{i}{\sqrt{8}}, \frac{i}{2}\right),\left(\frac{1}{\sqrt{2}}, 0, i\right) .
$$

Analogously as before, we write $(k, \ell, m)$ for

$$
\left(\frac{k}{\sqrt{8}}, \frac{\ell i}{\sqrt{8}}, \frac{m i}{2}\right) .
$$

The conformal vector is

$$
\frac{1}{2} x_{0,-1}^{2}-\frac{1}{2} x_{1,-1}^{2}+\frac{i}{2 \sqrt{2}} x_{1,-2}+\frac{1}{2} x_{2,-1}^{2} .
$$

The superconformal vectors are

$$
\begin{gathered}
G_{-3 / 2}^{-}=(0,4,2) x_{-1}(4,0,2), \\
G_{-3 / 2}^{+}=(0,4,-2) x_{-1}(-4,0,-2) .
\end{gathered}
$$


The fermionic labels will again be denoted by omitting the first coordinate: $(\ell, m)_{F}$. The fermionic identifications are:

$$
\begin{aligned}
& (2,2)_{F} \sim(2,-2)_{F} \sim(0,0)_{F} \\
& (1,1)_{F} \sim(1,-1)_{F} .
\end{aligned}
$$

A priori the lattice $\langle\sqrt{8}\rangle$ has 8 labels $\frac{k}{\sqrt{8}}, 0 \leq k \leq 7$, but the $G^{-}$definition together with (263) forces the MM identification of labels

$$
(1,1,1) \sim(-3,1,-1) \sim(-3,1,1) .
$$

The labels of the level $2 \mathrm{MM}$ are therefore

$$
\begin{aligned}
& (2 k, 0,0), 0 \leq k \leq 3 \\
& (2 k+1,1,1), 0 \leq k \leq 1 .
\end{aligned}
$$

The fusion rules are

$$
\begin{aligned}
& (k, 0,0) *(\ell, 0,0)=(k+\ell, 0,0), \\
& (k, 0,0) *(\ell, 1,1)=(k+\ell, 1,1), \\
& (k, 1,1) *(\ell, 1,1)=(k+\ell, 0,0)+(k+\ell+4,0,0),
\end{aligned}
$$

so the Verlinde algebra is simply

$$
\mathbb{Z}[a, b] /\left(a^{4}=1, b^{2}=a+a^{3}, a^{2} b=b\right)
$$

where $a=(2,0,0), b=(1,1,1)$.

One subtlety of the even level MM in comparison with odd level concerns signs. Since the $k$-coordinates of $G^{-}$and $G^{+}$are even, we can no longer use the $k$ coordinate of an element as an indication of parity ( $u$ and $G^{ \pm} u$ cannot have the same parity). Because of this, we must introduce odd fusion rules. There are various ways of doing this. For example, let the bottom states of $(2 k, 0,0),(1,1,1)$ and $(-1,1,1)$ be even. Then the fusion rules on level $\ell=0$ are even, as are the fusion rules combining levels 0 and 1 . The fusion rules

$$
(1,1,1) *(1,1,1) \mapsto(2,0,0),(1,1,1) *(-1,1,1) \mapsto(2,0,0)
$$

are even, the remaining fusion rules (adding 4 to the $k$-coordinate on the right hand side) are odd.

Now the $c$ fields of the MM are

$$
(0,0,0),(1,1,1),(2,2,2)
$$

and the $a$ fields are

$$
(0,0,0),(-1,1,-1),(-2,2,-2) \text {. }
$$

If we denote by $H_{1,2 k+1}$ the state space of label $(2 k+1,1,1), 0 \leq k \leq 1$, and by $H_{0,2 k}$ the state space of label $(2 k, 0,0), 0 \leq k \leq 3$, them the state space of the 4 -fold tensor product of the level 2 minimal model is

$$
\hat{\bigotimes}_{i=0}^{3}\left(\left(\underset{k_{i}=0}{\bigoplus} H_{0,2 k_{i}} \hat{\otimes} H_{0,2 k_{i}}^{*}\right) \oplus\left(\underset{k_{i}=0}{\bigoplus} H_{1,2 k_{i}+1} \hat{\otimes} H_{1,2 k_{i}+1}^{*}\right)\right) .
$$

The Gepner model is an orbifold with respect to the $\mathbb{Z} / 4$-group which acts by $i^{\ell}$ on products in (264) where the sum of the subscripts $2 k_{i}$ or $2 k_{i}+1$ is congruent to $\ell$ 
modulo 4. Therefore, the state space of the Gepner model is the sum over $\beta \in \mathbb{Z} / 4$ and $\alpha_{i} \in \mathbb{Z} / 4$,

$$
\sum_{i=0}^{3} \alpha_{i}=0 \in \mathbb{Z} / 4
$$

of

$$
\hat{\bigotimes}_{i=0}^{3}\left(\left(\underset{2 k_{i} \equiv \alpha_{i}}{\bigoplus \bmod 4} H_{0,2 k_{i}} \hat{\otimes} H_{0,2 k_{i}+2 \beta}^{*}\right) \oplus\left(\bigoplus_{2 k_{i}+1 \equiv \alpha_{i}} H_{1,2 k_{i}+1} \hat{\otimes} H_{1,2 k_{i}+1+2 \beta}^{*}\right)\right) .
$$

It is important to note that each summand (265) in which all the factors have the "odd" subscripts $1,2 k_{i}+1$ occurs twice in the orbifold state space.

If we write again $\ell$ for $(\ell, \ell, \ell)$ and $-\ell$ for $(-\ell, \ell,-\ell)$, then the critical $c c$ fields are chirally symmetric permutations of

$$
\begin{aligned}
& (2,2,0,0),(2,2,0,0) \\
& (2,1,1,0),(2,1,1,0) \\
& (1,1,1,1),(1,1,1,1) .
\end{aligned}
$$

Note that applying all the possible permutations to the fields (266), we obtain only 19 fields, while there should be 20 , which is the rank of $H^{11}(X)$ for a $K 3$-surface $X$. However, this is where the preceeding comment comes to play: the last field (266) corresponds to a term (265) where all the factors have odd subscripts, and hence there are two copies of that summand in the model, so the last field (266) occurs "twice".

By the fact that the Fermat quartic Gepner model has $N=(4,4)$ worldsheet supersymmetry (se e.g. 9, 51] and references therein), the spectral flow guarantees that the number of critical $a c$ fields is the same as the number of critical $c c$ fields. Concretely, the critical $a c$ fields are the permutations of

$$
\begin{aligned}
& (0,0,-2,-2),(2,2,0,0) \\
& (0,-1,-1,-2),(2,1,1,0) \\
& (-1,-1,-1,-1),(1,1,1,1) .
\end{aligned}
$$

As above, the last field (267) occurs in 2 copies, thus the rank of the space of critical $a c$ fields is also 20 .

We wish to investigate whether infinitesimal deformations along the fields (266), (267) exponentiate perturbatively. To this end, let us first see when the "cosettype scenario" occurs. This is sufficient to prove convergence in the present case. This is due to the fact that in the present theory, there is an even number of fermions, in which case it is well known by the boson-fermion correspondence that the correlation functions follow abelian fusion rules, and therefore Lemma 4 applies. To prove that the coset scenario occurs, let us look at the chiral $c$ fields

$$
u=(2,2,0,0),(2,1,1,0),(1,1,1,1)
$$

and study the singularities of

$$
G_{-1 / 2}^{-}(z)\left(G_{-1 / 2}^{-} u\right) .
$$


By Lemma 4, if (268) are non-singular, the obstructions vanish. The inner product is

$$
\begin{aligned}
& \left\langle(k, \ell, m),\left(k^{\prime}, \ell^{\prime}, m^{\prime}\right)\right\rangle=\frac{k k^{\prime}}{8}+\frac{\ell \ell^{\prime}}{8}-\frac{m m^{\prime}}{4}, \\
& w(k, \ell, m)=\frac{k^{2}}{16}+\frac{\ell(\ell+2)}{16}-\frac{m^{2}}{8} .
\end{aligned}
$$

Next, $(2,2,2)=(2,0,0)$,

$$
\begin{gathered}
G_{-1 / 2}^{-}(2,0,0)=(0,4,-2) x_{-1}(-2,0,-2), \\
G_{-1 / 2}^{-}(1,1,1)=(-3,1,-1) .
\end{gathered}
$$

if we again replace, to simplify notation, the symbol $G_{-1 / 2}^{-}$by $G$, then we have

The most singular $z$-power of $2(z) 2$ is $\frac{2 \cdot 2}{8}=\frac{1}{2}$,

For $G 2(z) G 2$, rename the rightmost $G 2$ as $(-2,2,0)$. We get

The most singular $z$-power of $G 2(z) G 2$ is $-1+\frac{(-2) \cdot(-2)}{8}=$ $-\frac{1}{2}$

The most singular $z$-power of $1(z) 1$ is 0 for the even fusion rule and $1 / 2$ for the odd fusion rule,

The most singular $z$-power of $G 1(z) 1$ is $\frac{-3}{8}+\frac{1}{8}+\frac{1}{4}=0$ for the even fusion rule, and $-1 / 2$ for the odd fusion rule,

The most singular $z$-power of $G 1(z) G 1$ is $\frac{9}{8}+\frac{1}{8}-\frac{1}{4}=1$ for the even fusion rule and $3 / 2$ for the odd fusion rule.

One therefore sees that for the field $u=(1,1,1,1)$, (268) is non-singular: In the case of the least favorable (odd) fusion rules, the most singular term appears to be -1 , coming from

$$
(G 1,1,1,1) \otimes(1, G 1,1,1) .
$$

However, this term cancels with

$$
(1, G 1,1,1) \otimes(G 1,1,1,1) .
$$

To see this, note that the last two coordinates do not enter the picture. We have an odd (resp. even) pair of pants $P_{-}$resp. $P_{+}$in the MM with input 1,1 . They add up to a pair of pants in $\mathrm{MM} \otimes \mathrm{MM}$. On (275), we have pairs of pants $P_{i} \in\left\{P_{-}, P_{+}\right\}$,

$$
\begin{aligned}
& P(G 1 \otimes 1) \otimes(1 \otimes G 1)= \\
& \left(P_{1} \otimes P_{2}\right)(G 1 \otimes 1) \otimes(1 \otimes G 1)= \\
& s P_{1}(G 1 \otimes 1) \otimes P_{2}(1 \otimes G 1)
\end{aligned}
$$

where $s$ is the sign of permuting $P_{2}$ past $G 1 \otimes 1$. Here we use the fact that 1 is even. On the other hand,

$$
\begin{aligned}
& P(1 \otimes G 1) \otimes(G 1 \otimes 1)= \\
& \left(P_{1} \otimes P_{2}\right)(1 \otimes G 1) \otimes(G 1 \otimes 1)= \\
& -s P_{1}(1 \otimes G 1) \otimes P_{2}(G 1 \otimes 1)
\end{aligned}
$$

(as $G 1$ is odd, so there is a - by permuting it with itself). From (69), the lowest term of $P_{i}(1 \otimes G 1)$ and $P_{i}(G 1 \otimes 1)$ have opposite signs, so (277) and (278) cancel out. 
The situation is simpler for $u=(2,2,0,0)$, in which case all the fusion rules are even, and the most singular term of

$$
(G 2 \otimes 2)(z)(2 \otimes G 2)
$$

appears to have most singular term $z^{-1}$. However, again note that 2 is even and $G 2$ is odd, so

$$
(G 2 \otimes s)(z)(2 \otimes G 2)=G 2(z) 2 \otimes 2(z) G 2,
$$

while

$$
(2 \otimes G 2)(z)(G 2 \otimes 2)=-2(z) G(z) \otimes G 2(z) 2 .
$$

Renaming $G 2$ as $(-2,2,0)$, the bottom descendant of both $G 2(z) 2$ and $2(z) G 2$ is $(0,2,0)$ with some coefficient, so (279) and (280) cancel out. Thus, the deformations along the first and last fields of (266) and (267) exponentiate.

The field $u=(2,1,1,0)$ is difficult to analyse, since in this case, (268) has singular channels and the coset-type scenario does not occur. We do not know how to calculate the obstruction directly in this case. It is however possible to present an indirect argument why these deformations exist.

In one precise formulation, the boson-fermion correspondence asserts that a tensor product of two copies of the 1-dimensional chiral fermion theory considered bosonically (= the level 2 parafermion) is an orbifold of the lattice theory $\langle 2\rangle$, by the $\mathbb{Z} / 2$-group whose generator acts on the lattice by sign.

This has an $N=2$-supersymmetric version. We tensor with two copies of the lattice theory associated with $\langle\sqrt{8}\rangle$, picking out the sector

$$
\left(\frac{m}{\sqrt{8}}, \frac{n}{\sqrt{8}}, \frac{p}{4}\right) \text { where } m \equiv n \equiv p \quad \bmod 2 .
$$

The fermionic currents of the individual coordinates are

$$
\psi_{-1 / 2,1}=(1)+(-1), \psi_{-1 / 2,2}=i((1)-(-1)),
$$

so the SUSY generators are

$$
\begin{gathered}
G_{-3 / 2,1}^{ \pm}=\left( \pm \frac{4}{\sqrt{8}}, 0\right) \otimes((1)+(-1)), G_{-3 / 2,2}^{ \pm}=\left(0, \pm \frac{4}{\sqrt{8}}\right) \otimes i((1)+(-1)), \\
G=G \cdot 1+G_{\cdot 2} .
\end{gathered}
$$

The $\mathbb{Z} / 2$ group acts trivially on the new lattice coordinate.

A note is due on the signs: To each state, we can assign a pair of parities, which will correspond to the parities of the 2 coordinates in the orbifold. This then also determines the sign of fusion rules.

Now consider our field as a tensor product of $(2,0)$ and $(1,1)$, each in a tensor product of two copies of the minimal models. Considering each of these factors as orbifold of the $N=2$-supersymmetric lattice theory, let us lift to the lattice theory:

$$
\begin{gathered}
(2,0) \mapsto\left(\frac{2}{\sqrt{8}}, 0\right) \otimes(0), \\
(1,1) \mapsto\left(\frac{1}{\sqrt{8}}, \frac{1}{\sqrt{8}}\right) \otimes((1 / 2)+(-1 / 2)) .
\end{gathered}
$$

Then the fields (284), (285) are $\mathbb{Z} / 2$-invariant. In the case of (284), we can proceed in the lift instead of the orbifold, because the fusion rules in the orbifold are abelian 
anyway. In the case of (285), the choice amounts to choosing a particular fusion rule. But now the point is that

$$
\begin{gathered}
G_{-1 / 2}^{-}(2,0) \mapsto\left(-\frac{2}{\sqrt{8}}, 0\right) \otimes((1)+(-1)), \\
G_{-1 / 2}^{-}(1,1) \mapsto\left(-\frac{3}{\sqrt{8}}, \frac{1}{\sqrt{8}}\right) \otimes((1 / 2)+(-1 / 2)), \\
G_{-1 / 2}^{-}(1,2) \mapsto\left(\frac{1}{\sqrt{8}},-\frac{3}{\sqrt{8}}\right) \otimes((1 / 2)+(-1 / 2)) .
\end{gathered}
$$

Thus, the left of $G_{-1 / 2}^{-} u$ is a sum of lattice labels!

Now the critical summands of the operator

$$
G_{-1 / 2}^{-}(u)\left(z_{k}\right) \ldots G_{-1 / 2}^{-}(u)\left(z_{1}\right)(u)(0)
$$

have $k=4 m$, and we have $2 m$ summands (286), and $m$ summands (287), (288), respectively. All

$$
\left(\begin{array}{c}
4 m \\
2 m, m, m
\end{array}\right)
$$

possibilities occur. It is the bottom (=label) term which we must compute in order to evaluate our obstruction. But by our sign discussion, when we swap a (287) term with a (288) term, the label summands cancel out. Now adding all such possible

$$
\left(\begin{array}{c}
4 m \\
2 m, m-1, m-1,2
\end{array}\right)
$$

pairs, all critical summands of (289) will occur with equal coefficients by symmetry, and hence also the bottom coefficient of (282) is 0 , thus showing the vanishing of our obstruction for this field lift to the lattice theory.

Since the field (285) is invariant under the $\mathbb{Z} / 2$-orbifolding (and although (284) isn't, the same conclusion holds when replacing it with its orbifold image), the entire perturbative deformation can also be orbifolded, yielding the desired deformation.

We thus conclude that for the Gepner model of the $K 3$ Fermat quartic, all the critical fields exponentiate to perturbative deformations.

\section{CONCLUSIONS AND Discussion}

In this paper, we have investigated perturbative deformations of CFT's by turning on a marginal $c c$ field, by the method of recursively updating the field along the deformation path. A certain algebraic obstruction arises. We work out some examples, including free field theories, and some $N=(2,2)$ supersymmetric Gepner models. In the $N=(2,2)$ case, in the case of a single $c c$ field, the obstruction we find can be made very explicit, and perhaps surprisingly, does not automatically vanish. By explicit computation, we found that the obstruction does not vanish for a particular critical $c c$ field in the Gepner model of the Fermat quintic 3-fold (we saw some indication, although not proof, that it may vanish for the field corresponding to adding the symmetric term xyztu to the superpotential, and for the unique critical $a c$ field). By comparison, the obstruction vanishes for the critical $c c$ fields and $a c$ fields Gepner model of the Fermat quartic $K 3$-surface. Our calculations are not completely physical in the sense that $c c$ fields are not real: real fields 
are obtained by adding in each case the complex-conjugate $a a$ field, in which case the calculation is more complicated and is not done here.

Assuming (as seems likely) that the real field case exhibits similar behaviour as we found, why are the $K 3$ and 3 -fold cases different, and what does the obstruction in the 3 -fold case indicate? In the $K 3$-case, our perturbative analysis conforms with the Aspinwall-Morrison [9] of the big moduli space of $K 3$ 's, and corresponding $(2,2)$ - (in fact, $(4,4)$-) CFT's, and also with the findings of Nahm and Wendland [51, 59].

In the 3-fold case, however, the straightforward perturbative construction of the deformed non-linear $\sigma$-model fails. This corresponds to the discussion of NemeschanskySen [52] of the renormalization of the non-linear $\sigma$-model. They expand around the 0 curvature tensor, but it seems natural to assume that similar phenomena would occur if we could expand around the Fermat quintic vacuum. Then 52 find that non-Ricci flat deformations must be added to the Lagrangian at higher orders of the deformation parameter in order to cancel the $\beta$ function. Therefore, if we want to do this perturbatively, fields must be present in the original (unperturbed) model which would correspond to non-Ricci flat deformation. No such fields are present in the Gepner model. (Even if we do not a priori assume that the marginal fields of the Gepner model correspond to Ricci flat deformations, we see that different fields are needed at higher order of the perturbation parameter, so there are not enough fields in the model.) More generally, ignoring for the moment the worldsheet SUSY, the bosonic superpartners are fields which are of weight 1 classically (as the classical non-linear $\sigma$-model Lagrangian is conformally invariant even in the non-Ricci flat case). A 1-loop correction arises in the quantum picture [4, indicating that the corresponding deformation fields must be of generalized weight (cf. [36, 37, 38, 39]). However, such fields are excluded in unitary CFT's, which is the reason why these deformations must be non-perturbative. One does not see this phenomenon on the level of the corresponding topological models, since these are invariant under varying the metric within the same cohomological class, and hence do not see the correction term 65. Also, it is worth noting that in the $K 3$-case, the $\beta$ function vanishes directly for the Ricci-flat metric by the $N=(4,4)$ supersymmetry ([5]), and hence the correction terms of 52 are not needed. Accordingly, we have found that the corresponding perturbative deformations exist.

From the point of view of mirror symmetry, mirror-symmetric families of hypersurfaces in toric varieties were proposed by Batyrev [10. In the case of the Fermat quintic, the exact mirror is a singular orbifold and the non-linear $\sigma$-model deformations corresponding to the Batyrev dual family exist perturbatively by our analysis. To obtain mirror candidates for the additional deformations, one uses crepant resolutions of the mirror orbifold (see [54] for a survey). In the $K 3$-case, this approach seems validated by the fact that the mirror orbifolds can indeed be viewed as a limit of non-singular $K 3$-surfaces $[6$. In the 3 -fold case, however, this is not so clear. The moduli spaces of Calabi-Yau 3-folds are not locally symmetric spaces. The crepant resolution is not unique even in the more restrictive category of algebraic varieties; different resolutions are merely related by flops. It is therefore not clear what the exact mirrors are of those deformations of the Fermat quntic 
where the deformation does not naturally occur in the Batyrev family, and resolution of singularities is needed. In other words, the McKay correspondence sees only "topological" invariants, and not the finer geometrical information present in the whole non-linear $\sigma$ model.

In [21, Fan, Jarvis and Ruan constructed exactly mathematically the $A$-models corresponding to Landau-Ginzburg orbifolds via Gromov-Witten theory applied to the Witten equation. Using mirror symmetry conjectures, this may be used to construct mathematically candidates of topological gravity-coupled $A$-models as well as $B$-models of Calabi-Yau varieties. Gromov-Witten theory, however, is a rich source of examples where such gravity-coupled topological models exist, while a full conformally invariant $(2,2)-\sigma$-model does not. For example, Gromov-Witten theory can produce highly non-trivial topological models for 0 -dimensional orbifolds (cf. [53, 40]).

Why does our analysis not contradict the calculation of Dixon [19] that the central charge does not change for deformation of any $N=2$ CFT along any linear combination of $a c$ and $c c$ fields? Zamolodchikov [67, 68] defined an invariant $c$ which is a non-decreasing function in a renormalization group flow in a 2-dimensional QFT, and is equal to the central charge in a conformal field theory. It may therefore appear that by [19, all infinitesimal deformations along critical $a c$ and $c c$ fields in an $N=2$-CFT exponentiate. However, we saw that when our obstruction occurs, additional counterterms corresponding to those of Nemeschansky-Sen are needed. This corresponds to non-perturbative corrections of the correlation function needed to fix $c$, and the functions [19] cannot be used directly in our case. 


\section{REFERENCES}

[1] I. Affleck, A.W. Ludwig: Universal Noninteger Ground State Degeneracy In Critical Quantum Systems, Phys. Rev. Lett. 67, 161 (1991)

[2] I. Affleck, A.W. Ludwig: Exact Conformal Field Theory Results On the Multichannel Kondo Effect: Single Fermion Green's Function, Selfenergy and Resistivity, JHEP 11 (2000) 21

[3] L. Alvarez-Gaume, S. Coleman and P. Ginsparg: Finiteness of Ricci flat $N=2$ supersymmetric $\sigma$-models. Comm. Math. Phys. 103 (1986), no. 3, 423-430

[4] L.Alvarez-Gaume, D.Z.Freedman: Kähler geometry and renormalization of supersymmetric $\sigma$-models, Phys. Rev. D 22 (1980) 846-853

[5] L.Alvarez-Gaume, P.Ginsparg: Finiteness of Ricci flat supersymmetric $\sigma$ models, Comm. Math. Phys. 102 (1985) 311-326

[6] M.T.Anderson: The $L^{2}$ structure of moduli spaces of Einstein metrics on 4-manifolds, Geom. Funct. Anal. 2 (1992) 29-89

[7] D.V.Anosov, A.A.Bolibruch: The Riemann-Hilbert problem, Aspects of Mathematics, E22, Friedr. Vieweg and Sohn, Braunschweig, 1994

[8] J.Ashkin and G.Teller: Statistics of two-dimensional lattices with four components, Phys. Rev. 64 (1943) 178-184

[9] P.S.Aspinwall, D.R.Morrison: String theory on K3 surfaces, in: Mirror symmetry, B.R.Greene and S.T.Yau eds., vol. II, 1994, 703-716

[10] V.V.Batyrev: Dual polyhedra and mirror symmetry for Calabi-Yau hypersurfaces in toric varieties, J. Alg. Geom. 3 (1994) 493-535

[11] R.J.Baxter: Eight-vertex model in lattice statistics, Phys. Rev. Lett. 26 (1971) 832-833

[12] P.Bouwknecht, D.Ridout: A Note on the Equality of Algebraic and Geometric D-Brane Charges in WZW, Journal H.E.P. 0405 (2004) 029

[13] R.Cohen, D.Gepner:, Interacting bosonic models and their solution, Mod. Phys. Lett. A6, 2249

[14] M.Dine, N.Seiberg, X.G.Wen and E.Witten: Non-perturbative effects on the string world sheet, Nucl. Phys. B 278 (1986) 769-969

[15] M.Dine, N.Seiberg, X.G.Wen and E.Witten: Non-perturbative effects on the string world sheet, Nucl. Phys. B 289 (1987) 319-363

[16] L.J.Dixon, V.S.Kaplunovsky, J.Louis: On effective field theories describing $(2,2)$ vacua of the heterotic string, Nucl. Phys. B 329 (1990) 27-82

[17] J.Ellis, C.Gomez, D.V.Nanopoulos and M.Quiros: World sheet instanton effects on no-scale structure, Phys. Lett. B 173 (1986), 59-64

[18] J.Distler, B.Greene: Some exact results on the superpotential from Calabi-Yau compactifications, Nucl. Phys. B 309 (1988) 295-316

[19] L.Dixon: Some worldsheet properties of superstring compactifications, on orbifolds and otherwise, in Superstrings, Unified Theories and Cosmology, Proc. ICTP Summer school, 1987, G. Furlan ed., World Scientific 1988, pp. 67-126

[20] M.R.Douglas, W. Taylor: The landscape of intersecting brane models, J. High Energy Phys. $0701(2007) 031$

[21] H.Fan, T.J.Jarvis, Y.Ruan: The Witten equation, mirror symmetry and quantum singularity theory, arXiv:0712.4021

[22] S. Fredenhagen, V. Schomerus: Branes on Group Manifolds, Gluon Condensates, and twisted K-theory, JHEP 104 (2001), 7

[23] D. Friedan: Nonlinear models in $2+\epsilon$ dimensions, Ann. Physics 163 (1985), no. 2, 318-419

[24] D.Gepner: Space-time supersymmetry in compactified string theory and superconformal models, Nucl. Phys. B 296 (1988) 757-778

[25] D.Gepner: Exactly solvable string compactifications on manifolds of $S U(N)$ holonomy, Phys. Letters B 199 (1987) 380

[26] M.Gerstenhaber: On the deformation of rings and algebras I-IV, Ann. of Math. 79 (1964) 59-103; 84 (1966) 1-19; 88 (1968) 1-34; 99 (1974) 257-276

[27] P.Ginsparg: Curiosities at $c=1$, Nucl. Phys. B 295 (1988) 153-170

[28] B.R.Greene: String theory on Calabi-Yau manifolds, hep-th/9702155

[29] B.R.Greene, M.R.Plesser: Duality in Calabi-Yau moduli space, Nucl. Phys. B 338 (1990) $15-37$ 
[30] B.R.Greene, C.Vafa, N.P.Warner: Calabi-Yau manifolds and renormalization group flows, Nucl. Phys. B 324 (1989) 371-390

[31] P.A.Griffin, O.F.Hernandez: Structure of irreducible $S U(2)$ parafermion modules derived vie the Feigin-Fuchs construction, Internat. Jour. Modern Phys. A 7 (1992) 1233-1265

[32] M.T.Grisaru, A.E.M.Van Den, D.Zanon: Four-loop $\beta$-function for the $N=1$ and $N=2$ supersymmetric non-linear sigma model in two dimensions, Phys. Lett. B 173 (1986) 423

[33] P.Hu, I.Kriz: Conformal field theory and elliptic cohomology, Adv. Math. 189 (2004) 325-412

[34] P.Hu, I.Kriz: Closed and open conformal field theories and their anomalies, Comm. Math. Phys. 254 (2005) 221-253

[35] P.Hu, I.Kriz: A mathematical formalism for the Kondo effect in WZW branes, hep-th/0508050

[36] Y.Z.Huang, J.Lepowsky, L.Zhang: Logarithmic tensor product theory for generalized modules for a conformal vertex algebra, Part I, math/0609833

[37] Y.Z.Huang, J.Lepowsky, L.Zhang: A logarithmic generalization of tensor product theory for modules for a vertex operator algebra, Int. Journal Math. 2006, math/0311235

[38] Y.Z.Huang, L.Kong: Full field algebras, QA/0511328

[39] Y.Z. Huang and A. Milas: Intertwining operator superalgebras and vertex tensor categories for superconformal algebras, II, Trans. Amer. Math. Soc. 354 (2002), 363-385.

[40] P.Johnson: Equivariant Gromov-Witten theory of one dimensional stacks, Ph.D. thesis, Univ. of Michigan, 2009

[41] S. Kachru, E. Witten: Computing The Complete Massless Spectrum of a Landau-Ginzburg Orbifold, Nucl. Phys. B 407 (1993) 637-666

[42] L.P.Kadanoff: Multicritical behavior of the Kosterlitz-Thouless Critical point, Ann. Phys. 120 (1979) 39-71

[43] L.P.Kadanoff, A.C.Brown: Correlation functions on the critical lines of the Baxter and Ashten-Teller models, Ann. Phys. 121 (1979) 318-342

[44] L.P.Kadanoff, F.J.Wegner: Some critical properties of the eight-vertex model, Phys. Review D 4 (1971) 3989-3993

[45] M.Kohmoto and L.P.Kadanoff: Lower bound RSRG approximation for a large system, J.Phys. A 13 (1980) 3339-3343

[46] I.Kriz: Some notes on the $N$-superconformal algebra, http://www.math.lsa.umich.edu/ikriz

[47] I.Kriz: On spin and modularity in conformal field theory, Ann. Sci. ENS 36 (2003) 57-112

[48] J.Maldacena, G.Moore, N.Seiberg: $D$-brane instantons and $K$-theory charges, JHEP 111 (2004), 62

[49] G. Moore: K-theory from a physical perspective, Topology, geometry and quantum field theory, London Math. Soc. Lecture Ser. 308, 194-234

[50] G. Mussardo, G. Sotkov, M. Stanishkov: $N=2$ superconformal minimal models, International Journal of Modern Physics A, vol.4, No.5 (1989) 1135-1206

[51] W.Nahm, K.Wendland: A hiker's guide to K3, Comm. Math. Phys. 216 (2001) 85-103

[52] D. Nemeschansky and A. Sen: Conformal invariance of supersymmetric $\sigma$-models on CalabiYau manifolds, Phys. Lett. B 178 (1986), no. 4, 365-369

[53] A. Okounkov, R. Pandharipande: The equivariant Gromov-Witten theory of $\mathbb{P}^{1}$, Ann. of Math. (2) 163 (2006), 561-605

[54] M.Reid: La Correspondence de McKay, 52eme annee, session de Novembre 1999, no.897, Asterisque 276 (2002) 53-72

[55] V. Schomerus: Lectures on branes in curved backgrounds, Class. Quant. Grav. 19 (2002), 5781

[56] G.Segal: The definition of conformal field theory, Topology, geometry and quantum field theory, London Math. Soc. Lecture Note Ser. 308, Cambridge University Press, 2004, 421577

[57] C. Vafa, N. Warner: Catastrophes and the Classification of Conformal Theories, Phys. Lett. B 218 (1989) 51

[58] F.J.Wegner: Corrections to scaling laws, Phys. Rev. B 5 (1972) 4529-4536

[59] K.Wendland: A family of SCFT's hosting all very attractive relatives to the $(2)^{4}$ Gepner model, JHEP 0603 (2006) 102

[60] K.G.Wilson: The renormalization group: Critical phenomena and the Kondo problem, Review of Mod. Phys. 47 (1975) 773-840

[61] K.G.Wilson: Non-Lagrangian models of current algebra, Phys. Rev. 179 (1969) 1499-1512 
[62] K.G.Wilson: Operator-product expansions and anomalous dimensions in the Thirring model, Phys. Rev. D 2 (1970) 1473-1493

[63] E. Witten: Phases of $N=2$ theories in two dimensions, Nucl. Phys. B 403 (1993), 159-222

[64] E. Witten: On the Landau-Ginzburg description of $N=2$ minimal models, Int. Jour. Modern. Phys. A 9 (1994) 4783-4800

[65] E.Witten: Topological Sigma Models, Comm. Math. Phys. 118 (1988) 411-449

[66] A.B. Zamolodchikov: Integrable Field Theory from Conformal Field Theory, Adv. stud. Pure Math. 19 (1989) 641-674

[67] A.B.Zamolodchikov: "Irreversibility" of the flux of the renormalization group in a 2D field theory, JETP Lett. 43 (1986) 730

[68] A.B.Zamolodchikov: Renormalization group and perturbation theory about fixed points in two-dimensional field theory, Sov. J.N.Phys. 46 (1987) 1090

[69] Y. Zhu: Modular invariance of characters of vertex operator algebras, J. Amer. Math. Soc. 9 (1996) 237-302 